\title{
Planck 2013 results. XI. All-sky model of thermal dust emission
}

Planck Collaboration: A. Abergel ${ }^{62}$, P. A. R. Ade ${ }^{90}$, N. Aghanim $^{62}$, M. I. R. Alves ${ }^{62}$, G. Aniano ${ }^{62}$, C. Armitage-Caplan ${ }^{95}$, M. Arnaud $^{75}$, M. Ashdown ${ }^{72,6}$, F. Atrio-Barandela ${ }^{19}$, J. Aumont ${ }^{62}$, C. Baccigalupi ${ }^{89}$, A. J. Banday ${ }^{98,9}$, R. B. Barreiro ${ }^{69}$, J. G. Bartlett ${ }^{1,70}$, E. Battaner ${ }^{100}$, K. Benabed ${ }^{63,97}$, A. Benoît ${ }^{60}$, A. Benoit-Lévy ${ }^{26,63,97}$, J.-P. Bernard ${ }^{98,9}$, M. Bersanelli3 ${ }^{36,51}$, P. Bielewicz ${ }^{98,9,89}$, J. Bobin $^{75}$, J. J. Bock ${ }^{70,10}$, A. Bonaldi ${ }^{71}$, J. R. Bond ${ }^{8}$, J. Borrill ${ }^{13,92}$, F. R. Bouchet ${ }^{63,97}$, F. Boulanger ${ }^{62}$, M. Bridges ${ }^{72,6,66}$, M. Bucher ${ }^{1}$, C. Burigana ${ }^{50,34}$, R. C. Butler ${ }^{50}$, J.-F. Cardoso ${ }^{76,1,63}$, A. Catalano ${ }^{77,74}$, A. Chamballu ${ }^{75,15,62}$, R.-R. Chary ${ }^{59}$, H. C. Chiang ${ }^{29,7}$, L.-Y Chiang ${ }^{65}$, P. R. Christensen ${ }^{84,39}$, S. Church ${ }^{94}$, M. Clemens ${ }^{46}$, D. L. Clements ${ }^{58}$, S. Colombi ${ }^{63,97}$, L. P. L. Colombo ${ }^{25,70},{\text { C. } \text { Combet }^{77} \text {, F. Couchot }}^{73}$, A. Coulais ${ }^{74}$, B. P. Crill ${ }^{70,85}$, A. Curto Co,69 $^{6}$,

F. Cuttaia ${ }^{50}$, L. Danese ${ }^{89}$, R. D. Davies ${ }^{71}$, R. J. Davis ${ }^{71}$, P. de Bernardis ${ }^{35}$, A. de Rosa ${ }^{50}$, G. de Zotti ${ }^{46,89}$, J. Delabrouille ${ }^{1}$, J.-M. Delouis ${ }^{63,97}$, F.-X. Désert ${ }^{55}$, C. Dickinson ${ }^{71}$, J. M. Diego ${ }^{69}$, H. Dole ${ }^{62,61}$, S. Donzelli ${ }^{51}$, O. Doré ${ }^{70,10}$, M. Douspis ${ }^{62}$, B. T. Draine ${ }^{87}$, X. Dupac $^{41}$, G. Efstathiou ${ }^{66}$, T. A. Enßlin ${ }^{80}$, H. K. Eriksen ${ }^{67}$, E. Falgarone ${ }^{74}$, F. Finelli ${ }^{50,52}$, O. Forni ${ }^{98,9}$, M. Frailis ${ }^{48}$, A. A. Fraisse ${ }^{29}$, E. Franceschi ${ }^{50}$, S. Galeotta ${ }^{48}$, K. Ganga $^{1}$,

T. Ghosh ${ }^{62}$, M. Giard ${ }^{98,9}$, G. Giardino ${ }^{42}$, Y. Giraud-Héraud ${ }^{1}$, J. González-Nuevo ${ }^{69,89}$, K. M. Górski ${ }^{70,101}$, S. Gratton ${ }^{72,66}$, A. Gregorio ${ }^{37,48,54}$, I. A. Grenier ${ }^{75}$, A. Gruppuso ${ }^{50}$, V. Guillet ${ }^{62}$, F. K. Hansen $^{67}$, D. Hanson ${ }^{81,70,8}$, D. L. Harrison ${ }^{66,72}$, G. Helou ${ }^{10}$, S. Henrot-Versillé ${ }^{73}$, C. Hernández-Monteagudo ${ }^{12,80}$, D. Herranz ${ }^{69}$, S. R. Hildebrandt ${ }^{10}$, E. Hivon ${ }^{63,97}$, M. Hobson ${ }^{6}$, W. A. Holmes ${ }^{70}$, A. Hornstrup ${ }^{16}$, W. Hovest ${ }^{80}$, K. M. Huffenberger ${ }^{27}$, A. H. Jaffe ${ }^{58}$, T. R. Jaffe ${ }^{98,9}$, J. Jewell ${ }^{70}$, G. Joncas ${ }^{18}$, W. C. Jones ${ }^{29}$, M. Juvela ${ }^{28}$, E. Keihänen ${ }^{28}$, R. Keskitalo ${ }^{23,13}$, T. S. Kisner ${ }^{79}$, J. Knoche ${ }^{80}$, L. Knox ${ }^{30}$, M. Kunz ${ }^{17,62,3}$, H. Kurki-Suonio ${ }^{28,44}$, G. Lagache ${ }^{62}$, A. Lähteenmäki ${ }^{2,44}$, J.-M. Lamarre ${ }^{74}$, A. Lasenby ${ }^{6,72}$, R. J. Laureijs ${ }^{42}$, C. R. Lawrence ${ }^{70}$, R. Leonardi ${ }^{41}$, J. León-Tavares ${ }^{43,2}$, J. Lesgourgues ${ }^{96,88}$, F. Levrier ${ }^{74}$, M. Liguori ${ }^{33}$, P. B. Lilje ${ }^{67}$,

M. Linden-Vørnle ${ }^{16}$, M. López-Caniego ${ }^{69}$, P. M. Lubin ${ }^{31}$, J. F. Macías-Pérez ${ }^{77}$, B. Maffei ${ }^{71}$, D. Maino ${ }^{36,51}$, N. Mandolesi ${ }^{50,5,34}$, M. Maris ${ }^{48}$, D. J. Marshall ${ }^{75}$, P. G. Martin ${ }^{8}$, E. Martínez-González ${ }^{69}$, S. Masi ${ }^{35}$, M. Massardi ${ }^{49}$, S. Matarrese ${ }^{33}$, F. Matthai ${ }^{80}$, P. Mazzotta ${ }^{38}$, P. McGehee ${ }^{59}$, A. Melchiorri ${ }^{35,53}$, L. Mendes ${ }^{41}$, A. Mennella ${ }^{36,51}$, M. Migliaccio ${ }^{66,72}$, S. Mitra ${ }^{57,70}$, M.-A. Miville-Deschênes ${ }^{62,8, *}$, A. Moneti ${ }^{63}$, L. Montier $^{98,9}$, G. Morgante ${ }^{50}$, D. Mortlock ${ }^{58}$, D. Munshi ${ }^{90}$, J. A. Murphy ${ }^{83}$, P. Naselsky ${ }^{84,39}$, F. Nati ${ }^{35}$, P. Natoli ${ }^{34,4,50}$, C. B. Netterfield ${ }^{21}$,

H. U. Nørgaard-Nielsen ${ }^{16}$, F. Noviello ${ }^{71}$, D. Novikov ${ }^{58}$, I. Novikov ${ }^{84}$, S. Osborne ${ }^{94}$, C. A. Oxborrow ${ }^{16}$, F. Paci ${ }^{89}$, L. Pagano ${ }^{35,53}$, F. Pajot ${ }^{62}$, R. Paladini ${ }^{59}$, D. Paoletti ${ }^{50,52}$, F. Pasian ${ }^{48}$, G. Patanchon ${ }^{1}$, O. Perdereau ${ }^{73}$, L. Perotto ${ }^{77}$, F. Perrotta ${ }^{89}$, F. Piacentini ${ }^{35}$, M. Piat ${ }^{1}$, E. Pierpaoli ${ }^{25}$ D. Pietrobon ${ }^{70}$, S. Plaszczynski ${ }^{73}$, E. Pointecouteau ${ }^{98,9}$, G. Polenta ${ }^{4,47}$, N. Ponthieu ${ }^{62,55}$, L. Popa ${ }^{64}$, T. Poutanen ${ }^{44,28,2}$, G. W. Pratt ${ }^{75}$,

G. Prézeau ${ }^{10,70}$, S. Prunet ${ }^{63,97}$, J.-L. Puget ${ }^{62}$, J. P. Rachen ${ }^{22,80}$, W. T. Reach ${ }^{99}$, R. Rebolo ${ }^{68,14,40}$, M. Reinecke ${ }^{80}$, M. Remazeilles ${ }^{71,62,1}$, C. Renault ${ }^{77}$, S. Ricciardi ${ }^{50}$, T. Riller ${ }^{80}$, I. Ristorcelli ${ }^{88,9}$, G. Rocha ${ }^{70,10}$, C. Rosset $^{1}$, G. Roudier ${ }^{1,74,70}$, M. Rowan-Robinson ${ }^{58}$, J. A. Rubiño-Martín ${ }^{68,40}$,

B. Rusholme ${ }^{59}$, M. Sandri ${ }^{50}$, D. Santos ${ }^{77}$, G. Savini ${ }^{86}$, D. Scott ${ }^{24}$, M. D. Seiffert ${ }^{70,10}$, E. P. S. Shellard ${ }^{11}$, L. D. Spencer ${ }^{90}$, J.-L. Starck ${ }^{75}$, V. Stolyarov ${ }^{6,72,93}$, R. Stompor ${ }^{1}$, R. Sudiwala ${ }^{90}$, R. Sunyaev ${ }^{80,91}$, F. Sureau ${ }^{75}$, D. Sutton ${ }^{66,72}$, A.-S. Suur-Uski ${ }^{28,44}$, J.-F. Sygnet ${ }^{63}$, J. A. Tauber ${ }^{42}$,

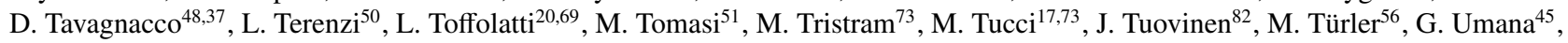

L. Valenziano ${ }^{50}$, J. Valiviita ${ }^{44,28,67}$, B. Van Tent ${ }^{78}$, L. Verstraete ${ }^{62}$, P. Vielva ${ }^{69}$, F. Villa $^{50}$, N. Vittorio $^{38}$, L. A. Wade ${ }^{70}$, B. D. Wandelt ${ }^{63,97,32}$, N. Welikala ${ }^{1}$, N. Ysard ${ }^{28}$, D. Yvon ${ }^{15}$, A. Zacchei ${ }^{48}$, and A. Zonca ${ }^{31}$

(Affiliations can be found after the references)

Received 4 December 2013 / Accepted 4 September 2014

\section{ABSTRACT}

This paper presents an all-sky model of dust emission from the Planck 353, 545, and $857 \mathrm{GHz}$, and IRAS $100 \mu$ m data. Using a modified blackbody fit to the data we present all-sky maps of the dust optical depth, temperature, and spectral index over the 353-3000 GHz range. This model is a good representation of the IRAS and Planck data at $5^{\prime}$ between 353 and $3000 \mathrm{GHz}(850$ and $100 \mu \mathrm{m})$. It shows variations of the order of $30 \%$ compared with the widely-used model of Finkbeiner, Davis, and Schlegel. The Planck data allow us to estimate the dust temperature uniformly over the whole sky, down to an angular resolution of $5^{\prime}$, providing an improved estimate of the dust optical depth compared to previous all-sky dust model, especially in high-contrast molecular regions where the dust temperature varies strongly at small scales in response to dust evolution, extinction, and/or local production of heating photons. An increase of the dust opacity at $353 \mathrm{GHz}, \tau_{353} / N_{\mathrm{H}}$, from the diffuse to the denser interstellar medium (ISM) is reported. It is associated with a decrease in the observed dust temperature, $T_{\mathrm{obs}}$, that could be due at least in part to the increased dust opacity. We also report an excess of dust emission at $\mathrm{H}$ I column densities lower than $10^{20} \mathrm{~cm}^{-2}$ that could be the signature of dust in the warm ionized medium. In the diffuse ISM at high Galactic latitude, we report an anticorrelation between $\tau_{353} / N_{\mathrm{H}}$ and $T_{\mathrm{obs}}$ while the dust specific luminosity, i.e., the total dust emission integrated over frequency (the radiance) per hydrogen atom, stays about constant, confirming one of the Planck Early Results obtained on selected fields. This effect is compatible with the view that, in the diffuse ISM, $T_{\text {obs }}$ responds to spatial variations of the dust opacity, due to variations of dust properties, in addition to (small) variations of the radiation field strength. The implication is that in the diffuse high-latitude ISM $\tau_{353}$ is not as reliable a tracer of dust column density as we conclude it is in molecular clouds where the correlation of $\tau_{353}$ with dust extinction estimated using colour excess measurements on stars is strong. To estimate Galactic $E(B-V)$ in extragalactic fields at high latitude we develop a new method based on the thermal dust radiance, instead of the dust optical depth, calibrated to $E(B-V)$ using reddening measurements of quasars deduced from Sloan Digital Sky Survey data.

Key words. methods: data analysis - ISM: general - dust, extinction - infrared: ISM - submillimeter: ISM - opacity

Corresponding author: Marc-Antoine Miville-Deschênes, e-mail: mamd@ias.u-psud.fr 


\section{Introduction}

This paper, one of a set associated with the 2013 release of data from the Planck $^{1}$ mission (Planck Collaboration I 2014), presents a new parametrization of dust emission that covers the whole sky, at $5^{\prime}$ resolution, based on data from 353 to $3000 \mathrm{GHz}$ (100 to $850 \mu \mathrm{m})$.

Because it is well mixed with the gas and because of its direct reaction to UV photons from stars, dust is a great tracer of the interstellar medium (ISM) and of star formation activity. On the other hand, for many studies in extragalactic astrophysics and cosmology, Galactic interstellar dust is a nuisance, a source of extinction and reddening for UV to near-infrared observations and a contaminating emission in the infrared to millimetre wavelengths. Thanks to the sensitivity, spectral coverage, and angular resolution of Planck, this model of dust emission brings new constraints on the dust spectral energy distribution (SED), on its variations across the sky, and on the relationships between dust emission, dust extinction, and gas column density. In particular, this model of dust emission provides a new map of dust extinction at $5^{\prime}$ resolution, aimed at helping extragalactic studies.

The emission in the submillimetre range arises from the bigger dust grains that are in thermal equilibrium with the ambient radiation field. Thermal dust emission is influenced by a combination of the dust column density, radiation field strength, and dust properties (size distribution, chemical composition, and the grain structure). When the effect of the radiation field can be estimated (using the dust temperature as a probe) and the dust properties assumed, the dust optical depth is possibly the most reliable tracer of interstellar column density, and therefore of mass for objects at known distances. Dust optical depth is used to estimate the mass of interstellar clumps and cores (Ossenkopf \& Henning 1994) in particular with the higher resolution Herschel data (Launhardt et al. 2013), to study the statistical properties of the ISM structure and its link with gravity, interstellar turbulence, and stellar feedback (Peretto et al. 2012; Kainulainen et al. 2013), and as a way to sample the mass of the ISM in general (Planck Collaboration XIX 2011). The accuracy of these determinations depends on, among other things, the frequency range over which the dust spectrum is observed. The combination of Planck and IRAS data offers a new view on interstellar dust by allowing us to sample the dust spectrum from the Wien to the Rayleigh-Jeans sides, at $5^{\prime}$ resolution over the whole sky.

Dust emission, with extinction and polarization, is a key element to constrain the properties of interstellar dust (Draine \& Li 2007; Compiègne et al. 2011). The dust emissivity (i.e., the amount of emission per unit of gas column density) and the shape of the dust SED provide information on the nature of the dust particles, in particular their structure, composition, and abundance, related to the dust-to-gas ratio.

Changes in the dust emissivity and the shape of the dust SED can be related to dust evolutionary processes. Interstellar dust grains are thought to be the seeds from which larger particles form in the ISM, up to planetesimals in circumstellar environments (Brauer et al. 2008; Beckwith et al. 2000; Birnstiel et al. 2012). This growth of solids can be followed in earlier phases of the star-formation process, at the protostellar phase and even before, in molecular clouds and in the diffuse ISM.

\footnotetext{
1 Planck (http://www.esa.int/Planck) is a project of the European Space Agency (ESA) with instruments provided by two scientific consortia funded by ESA member states (in particular the lead countries France and Italy), with contributions from NASA (USA) and telescope reflectors provided by a collaboration between ESA and a scientific consortium led and funded by Denmark.
}

Many studies have revealed increases of the dust emissivity with (column) density in molecular clouds accompanied by a decrease in dust temperature (Stepnik et al. 2003; Schnee et al. 2008; Planck Collaboration XXV 2011; Arab et al. 2012; Martin et al. 2012; Roy et al. 2013). One explanation is that grain structure is changing through aggregation of smaller particles, enhancing the opacity (Köhler et al. 2011). Planck's spectral coverage allows us to model the big grain thermal emission, in particular its spectral index that is related to the grain composition and structure (Ormel et al. 2011; Meny et al. 2007; Köhler et al. 2012). Because of its full-sky coverage, Planck can also reveal variations of the dust SED with environment, enabling us to better understand the evolutionary track of dust grains through the ISM phases.

Dust emission is one of the major foregrounds hampering the study of the cosmic microwave background (CMB). The thermal dust emission peaks at a frequency close to $2000 \mathrm{GHz}$ but its emission is still a fair fraction of the CMB anisotropies in the 20-200 GHz range where they are measured. This is even more the case in polarization (Miville-Deschênes 2011). The model of dust emission proposed by Finkbeiner et al. (1999) based on data from previous satellite missions (IRAS and COBE) made an important contribution to the field, in guiding the design of $\mathrm{CMB}$ experiments and in helping the data analysis by providing a spatial template and a spectral dependence of the dust emission at CMB frequencies. It is still the basis of recent models of Galactic foreground emission (Delabrouille et al. 2013). With its frequency coverage that bridges the gap between IRAS and the CMB range, its high sensitivity, and its better angular resolution, Planck offers the opportunity to develop a new model of thermal dust emission.

Estimating reddening and extinction by foreground interstellar dust is a major issue for observations of extragalactic objects in the UV to near-infrared range. Major efforts have been made toward producing sky maps that provide a way to correct for the chromatic extinction of light by Galactic interstellar dust on any line of sight. First Burstein \& Heiles (1978) used H I as a proxy for dust extinction by correlating integrated $21 \mathrm{~cm}$ line emission with extinction estimated from galaxy counts. It was subsequently discovered that $\mathrm{H} \mathrm{I}$ is not a reliable tracer of total column density $N_{\mathrm{H}}$ for $N_{\mathrm{H} \text { I }}$ greater than a few $10^{20} \mathrm{~cm}^{-2}$ due to molecular gas contributions (Lebrun et al. 1982; Boulanger \& Pérault 1988; Désert et al. 1988; Heiles et al. 1988; Blitz et al. 1990; Reach et al. 1994; Boulanger et al. 1996). It was then proposed to use dust emission as a more direct way to estimate dust extinction. By combining 100, 140, and $240 \mu \mathrm{m}$ data (DIRBE and IRAS) Schlegel et al. (1998) produced an all-sky map of dust optical depth at $100 \mu \mathrm{m}$ that was then calibrated into dust reddening by correlating with colour excesses measured for galaxies. The work presented here is the direct continuation of these studies. Like Schlegel et al. (1998) we also propose a map of $E(B-V)$ based on a model of dust emission calibrated using colour excess measurements of extragalactic objects, here quasars.

The paper is organized as follows. The data used and the preprocessing steps are presented in Sect. 2. The model of the dust emission, SED fit methodology, the exploration of potential biases, and the all-sky maps of dust parameters are described in Sect. 3. The results of the Galactic dust model are analysed in Sect. 4. Sections 5 and 6 describe specifically how the dust emission model compares to other tracers of column density. The Planck dust products, the dust model maps, and the $E(B-V)$ map aimed at helping extragalactic studies to estimate Galactic extinction are detailed in Sect. 7 and compared with similar products in the literature. Concluding remarks are given in Sect. 8. 


\section{Data and preprocessing}

The analysis presented here relies on the combination of the Planck data from the HFI instrument at 857,545 , and $353 \mathrm{GHz}$ (respectively 350, 550, and $850 \mu \mathrm{m}$ ) with the IRAS $100 \mu \mathrm{m}$ $(3000 \mathrm{GHz})$ data.

\subsection{Planck data}

For Planck we used the HFI 2013 delivery maps (Planck Collaboration VI 2014), corrected for zodiacal emission (ZE see Planck Collaboration XIV 2014). Each map was smoothed to a common resolution of $5^{\prime}$, assuming a Gaussian beam ${ }^{2}$. The $353 \mathrm{GHz}$ map, natively built in units of $K_{\mathrm{CMB}}$, was transformed to $\mathrm{MJy} \mathrm{sr}^{-1}$ using the conversion factor given by Planck Collaboration IX (2014). The CMB anisotropies map provided by the SMICA algorithm (Planck Collaboration XII 2014), which has an angular resolution of $5^{\prime}$, was removed from each Planck HFI map.

As shown in Planck Collaboration XIII (2014), ${ }^{12} \mathrm{CO}$ and ${ }^{13} \mathrm{CO}$ rotational lines fall in each of the Planck HFI filters, except at $143 \mathrm{GHz}$. At 857 and $545 \mathrm{GHz}$ the $\mathrm{CO}$ lines $(J=5 \rightarrow 4$ and $J=4 \rightarrow 3$, respectively) are very faint compared to the dust emission and they are not considered here. On the other hand, emission from the ${ }^{12} \mathrm{CO} J=3 \rightarrow 2$ line was detected in the $353 \mathrm{GHz}$ band (Planck Collaboration XIII 2014). Nevertheless, this emission is still faint compared to the dust emission whereas the noise on the Planck CO emission estimate in the $353 \mathrm{GHz}$ band is quite high (see Planck Collaboration XIII 2014 for details). The detection of the ${ }^{12} \mathrm{CO} J=3 \rightarrow 2$ line emission by Planck is above $3 \sigma$ for only $2.6 \%$ of the sky. When detected above $3 \sigma$, this emission is on average $2 \%$ of the $353 \mathrm{GHz}$ specific intensity. It contributes $5 \%$ or more of the $353 \mathrm{GHz}$ specific intensity for only $0.3 \%$ of the sky. Given such a relatively small contribution we did not subtract $\mathrm{CO}$ emission from the data so as not to compromise the $353 \mathrm{GHz}$ map through the adverse impact of the noise of the ${ }^{12} \mathrm{CO} J=3 \rightarrow 2$ product.

\subsection{The $100 \mu \mathrm{m}$ map}

The $100 \mu \mathrm{m}$ map used in this analysis is a combination of the IRIS map (Miville-Deschênes \& Lagache 2005) and the map of Schlegel et al. (1998, hereafter SFD), both projected on the HEALPix ${ }^{3}$ grid (Górski et al. 2005) at $N_{\text {side }}=2048$. Both IRIS and SFD maps were built by combining IRAS and DIRBE $100 \mu \mathrm{m}$ data. Nevertheless these two maps show differences at large scales due to the different assumptions used for the ZE removal. Miville-Deschênes \& Lagache (2005) used the DIRBE $100 \mu \mathrm{m}$ map from which ZE was removed by the DIRBE team, using the model of Kelsall et al. (1998). On the other hand, Schlegel et al. (1998) used their own empirical approach to remove ZE based on a scaling of the DIRBE $25 \mu \mathrm{m}$ data. Because it is based on data and not on a model, the SFD correction is closer to the complex structure of the $\mathrm{ZE}$ and provides a better result. This can be assessed by looking at the correlation of the IRIS and SFD maps with H I in the diffuse areas of the sky $\left(1<N_{\mathrm{HI}}<2 \times 10^{20} \mathrm{~cm}^{-2}\right)$, as detailed in Appendix A.1. The uncertainty of the slope of the correlation with $N_{\mathrm{HI}}$ and the standard deviation of the residual is about $30 \%$ lower for the SFD

\footnotetext{
2 Each map was smoothed using a Gaussian beam of FWHM, $f_{s}$, that complements the native FWHM, $f_{i}$ (Table 1), of the map to bring it to $5^{\prime}: f_{s}=\sqrt{5^{2}-f_{i}^{2}}$.

3 http://healpix. sourceforge.net
}

map compared to the IRIS map. For that reason (and others described in Appendix A.1) we favour the use of the SFD map at large scales.

At scales smaller than $30^{\prime}$, the IRIS map has several advantages over the SFD map ${ }^{4}$. The IRIS map is at the original angular resolution (4!3) of the IRAS data while SFD smoothed the map to 6!1. IRIS also benefits from a non-linear gain correction that is coherent for point sources and diffuse emission. Finally point sources were kept in the IRIS map whereas SFD removed some of them (mostly galaxies but also ISM clumps). To combine the advantages of the two maps, we built a $100 \mu \mathrm{m}$ map, $I_{100}$, that is compatible with SFD at scales larger than $30^{\prime}$ and compatible with IRIS at smaller scales:

$I_{100}=I_{\mathrm{IRIS}}-I_{\mathrm{IRIS}} \otimes f_{\mathrm{IRIS}}^{30}+I_{\mathrm{SFD}} \otimes f_{\mathrm{SFD}}^{30}$,

where $I_{\mathrm{IRIS}}$ and $I_{\mathrm{SFD}}$ are, respectively, the IRIS and the SFD maps, and $f_{i}^{30}$ is the complementary Gaussian kernel needed to bring the maps to $30^{\prime}$ resolution.

\subsection{Zero level}

The fit of the dust emission requires that the specific intensity at each frequency and at each sky position is free of any other emission. In particular the zero level of each map should be set in such a way that it contains only Galactic dust emission. In order to set the zero level of the maps to a meaningful Galactic reference we applied a method based on a correlation with $\mathrm{HI}$, as described in Planck Collaboration VIII (2014).

Some precautions need to be taken here because the ratio of dust to H I emission might vary locally due to variations of the radiation field or of the dust optical properties. Locally the $21 \mathrm{~cm}$ emission might not be a perfect tracer of the column density due to H I self-absorption effects or to the presence of ionized or molecular gas. Nevertheless, the correlation between dust and H I emission is known to be tight in the diffuse ISM where most of the gas is atomic. This correlation has been used several times to establish the dust SED (Boulanger et al. 1996; Planck Collaboration XXIV 2011), to isolate the cosmic infrared background (CIB; Puget et al. 1996; Planck Collaboration XVIII 2011; Pénin et al. 2012), and to establish a Galactic reference for dust maps (Burstein \& Heiles 1978; Schlegel et al. 1998).

The excess of dust emission with respect to the H I correlation has been used to reveal gas in molecular form, even in regions where CO emission was not detected (Désert et al. 1988; Blitz et al. 1990; Reach et al. 1998; Planck Collaboration XIX 2011). Such an excess can be observed at column densities as low as $N_{\mathrm{HI}}=2 \times 10^{20} \mathrm{~cm}^{-2}$. Using this as an upper limit for our correlation studies also ensures that self-absorption in the $21 \mathrm{~cm}$ line emission is not important. Note that this is also below the threshold at which significant $\mathrm{H}_{2}$ is seen in the diffuse ISM (Gillmon et al. 2006; Wakker 2006; Rachford et al. 2002, 2009).

To estimate the Galactic reference of the IRAS and Planck data, the maps were correlated against the $21 \mathrm{~cm} \mathrm{LAB}$ data (Kalberla et al. 2005), integrated in velocity. The ranges are referred to as LVC, low velocity gas with $\left|v_{\mathrm{LSR}}\right|<35 \mathrm{~km} \mathrm{~s}^{-1}$, and IVC, intermediate velocity gas with $35<\left|v_{\text {LSR }}\right|<70 \mathrm{~km} \mathrm{~s}^{-1}$ (Albert \& Danly 2004). HVC, high-velocity clouds with $\left|v_{\text {LSR }}\right|>$ $70 \mathrm{~km} \mathrm{~s}^{-1}$ are excluded. For LVC and IVC separately, column density maps $N_{\mathrm{H}}$ assuming optically thin emission are given

4 It is at $30^{\prime}$ that both maps match in power - see Fig. 15 of Miville-Deschênes \& Lagache (2005). This scale is close to the resolution of the DIRBE data $\left(42^{\prime}\right)$ that were used in both products to set the large-scale emission. 

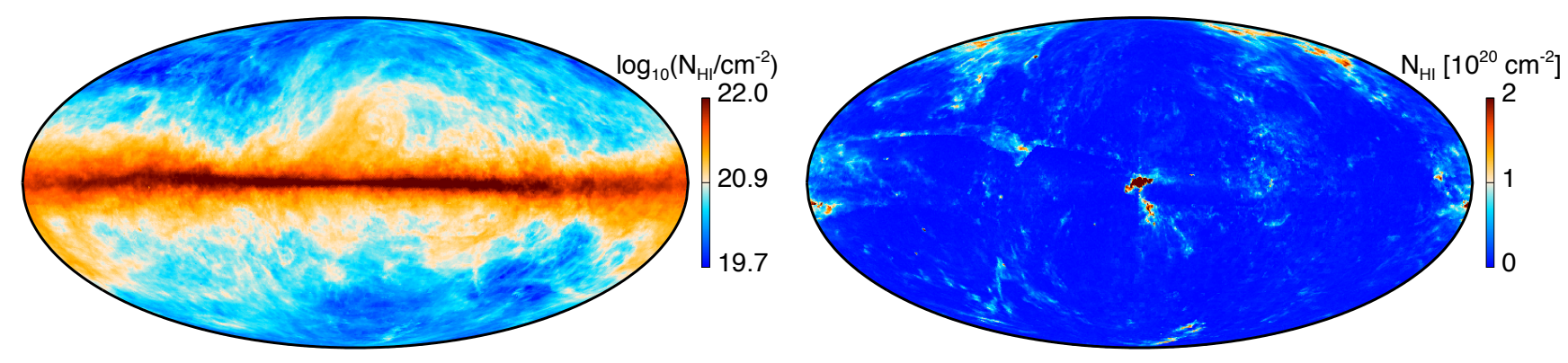

Fig. 1. All-sky Mollweide projections of H I maps used in the determination of the offsets. The centre of the map is toward the Galactic centre. Left: H I column density of low velocity clouds (LVC). Right: intermediate velocity clouds (IVC). See text.

Table 1. Properties of the IRAS and Planck maps from which ZE has been removed.

\begin{tabular}{cccccc}
\hline \hline $\begin{array}{c}v \\
{[\mathrm{GHz}]}\end{array}$ & $\begin{array}{c}\lambda \\
{[\mu \mathrm{m}]}\end{array}$ & $\begin{array}{c}F W H M \\
{[\operatorname{arcmin}]}\end{array}$ & $\begin{array}{c}\text { Offset } \\
{\left[\mathrm{MJy} \mathrm{sr}^{-1}\right]}\end{array}$ & $\begin{array}{c}\text { Dipole } \\
{\left[\mathrm{MJy} \mathrm{sr}^{-1}\right]}\end{array}$ & $\begin{array}{c}c_{v} \\
{[\%]}\end{array}$ \\
\hline 3000 & 100 & 4.3 & $-0.174 \pm 0.005$ & - & 13.6 \\
857 & 350 & 4.63 & $0.093 \pm 0.009$ & - & 10.0 \\
545 & 550 & 4.84 & $0.095 \pm 0.014$ & $0.0148 \pm 0.0001$ & 10.0 \\
353 & 850 & 4.86 & $0.085 \pm 0.006$ & $-0.0089 \pm 0.0001$ & 1.2 \\
\hline
\end{tabular}

Notes. Column 1: frequency. Column 2: wavelength. Column 3: angular resolution (see Planck Collaboration VII 2014). Column 4: offset (and its uncertainty $\delta O_{v}$ ) removed from the maps to adjust them to a coherent Galactic zero level (see Planck Collaboration VIII 2014). Column 5: amplitude of the residual dipole removed. The residual dipole removed at 353 and $545 \mathrm{GHz}$ is oriented toward $l=263^{\circ} .99, b=48^{\circ} .26$, the direction of the solar dipole estimated using WMAP data (Hinshaw et al. 2009). Column 6: calibration uncertainty.

in Fig. 1 in the all-sky Mollweide equal-area projection and in Fig. 2 in a complementary polar orthographic projection ${ }^{5}$.

For the correlation, all data sets were convolved to $1^{\circ}$ resolution and projected on an $N_{\text {side }}=128$ grid. The correlation was performed using only pixels with the LVC $N_{\mathrm{HI}}<2 \times 10^{20} \mathrm{~cm}^{-2}$, discarding pixels with detected IVC above $0.1 \times 10^{20} \mathrm{~cm}^{-2}$. The resulting area covers $11.5 \%$ of the sky, corresponding to more than $4700 \mathrm{deg}^{2}$. This mask is presented in both the all-sky view in Fig. 3 (left panel) and the polar view in Fig. 4. We refer to this throughout as the "low $N_{\mathrm{HI}}$ mask".

The correlations are shown in Fig. 5, left. The values of the offset that were removed from the 3000 and $857 \mathrm{GHz}$ maps are given in Table 1. We have checked that the offsets estimated in that way are not sensitive to the resolution of the H I data or to the area of the sky selected. We have also checked that the assumption that the $21 \mathrm{~cm}$ emission is optically thin does not introduce a significant bias in the analysis. For example, on assuming $T_{\text {spin }}=80 \mathrm{~K}$ in converting $21 \mathrm{~cm}$ emission to $N_{\mathrm{HI}}$ (Lockman \& Condon 2005), the changes in the offsets at 857 and $3000 \mathrm{GHz}$ are only about $0.02 \mathrm{MJy} \mathrm{sr}^{-1}$. This is as expected because in the LAB data for the diffuse areas of the sky considered here values of the $21 \mathrm{~cm}$ line brightness temperature higher than $10 \mathrm{~K}$ are exceptional. Compatible offsets, within the quoted uncertainties, were found using 16' Galactic All Sky Survey $21 \mathrm{~cm}$ data (McClure-Griffiths et al. 2009) of the area around the Galactic south pole (Planck Collaboration Int. XVII 2014) and 9' data obtained on smaller regions in the northern sky at the Green Bank Telescope (Planck Collaboration XXIV 2011). Finally, in Fig. 5 we note a systematic excess of the dust emission at 857 and $3000 \mathrm{GHz}$ with respect to the correlation at the lowest $N_{\mathrm{HI}}$.

\footnotetext{
5 Each of these projections (Calabretta \& Greisen 2002) covers the whole sky, but here we use the terminology "all-sky" and "polar" as shorthand for the two projections. The polar view is most useful for the high-latitude sky, whereas the all-sky view is best for intermediate to low latitudes.
}

This is also seen at 545 and $353 \mathrm{GHz}$ and it is discussed further in Sect. 5.4.

The correlation of dust emission between Planck frequencies is observed to be tight (the correlation of Planck is less tight with the IRAS $3000 \mathrm{GHz}$ map). We took advantage of this to estimate more precisely the Galactic zero level of the 353 and $545 \mathrm{GHz}$ channels. They were obtained by correlation with the $857 \mathrm{GHz}$ map on a larger mask with total LVC plus IVC $N_{\mathrm{H}}<3 \times 10^{20} \mathrm{~cm}^{-2}$ (Fig. 3, right panel). These correlations are shown in Fig. 5 (right). The offset values obtained in this way (see Table 1) are compatible within $1 \sigma$ with the offset values deduced from the $\mathrm{H}$ I correlation in the smaller mask $(0.104$ and $0.088 \mathrm{MJy} \mathrm{sr}^{-1}$ respectively at 545 and $353 \mathrm{GHz}$ ). At these frequencies we favour the offset obtained with the correlation with $857 \mathrm{GHz}$ as it minimizes the aforementioned effects in the correlation between dust and gas emission.

In the process, faint dipole residuals were identified in the 353 and $545 \mathrm{GHz}$ maps. The orientation of these residual dipoles coincides with the solar dipole and their amplitudes (see Table 1) corresponds to $+7.6 \%$ and $-0.9 \%$ of the solar dipole amplitude at 545 and $353 \mathrm{GHz}$ respectively, which is within the calibration uncertainties at these frequencies. They were removed prior to the dust SED fit.

\section{Model of the dust emission}

\subsection{Dust emission observed by Planck}

The emission from interstellar dust in the far-infrared (FIR) to millimetre range is dominated by the emission from the biggest grains that are in thermal equilibrium with the local radiation field. Many studies and reviews have been dedicated to this subject (e.g., Draine 2003; Draine \& Li 2007; Compiègne et al. 2011). 

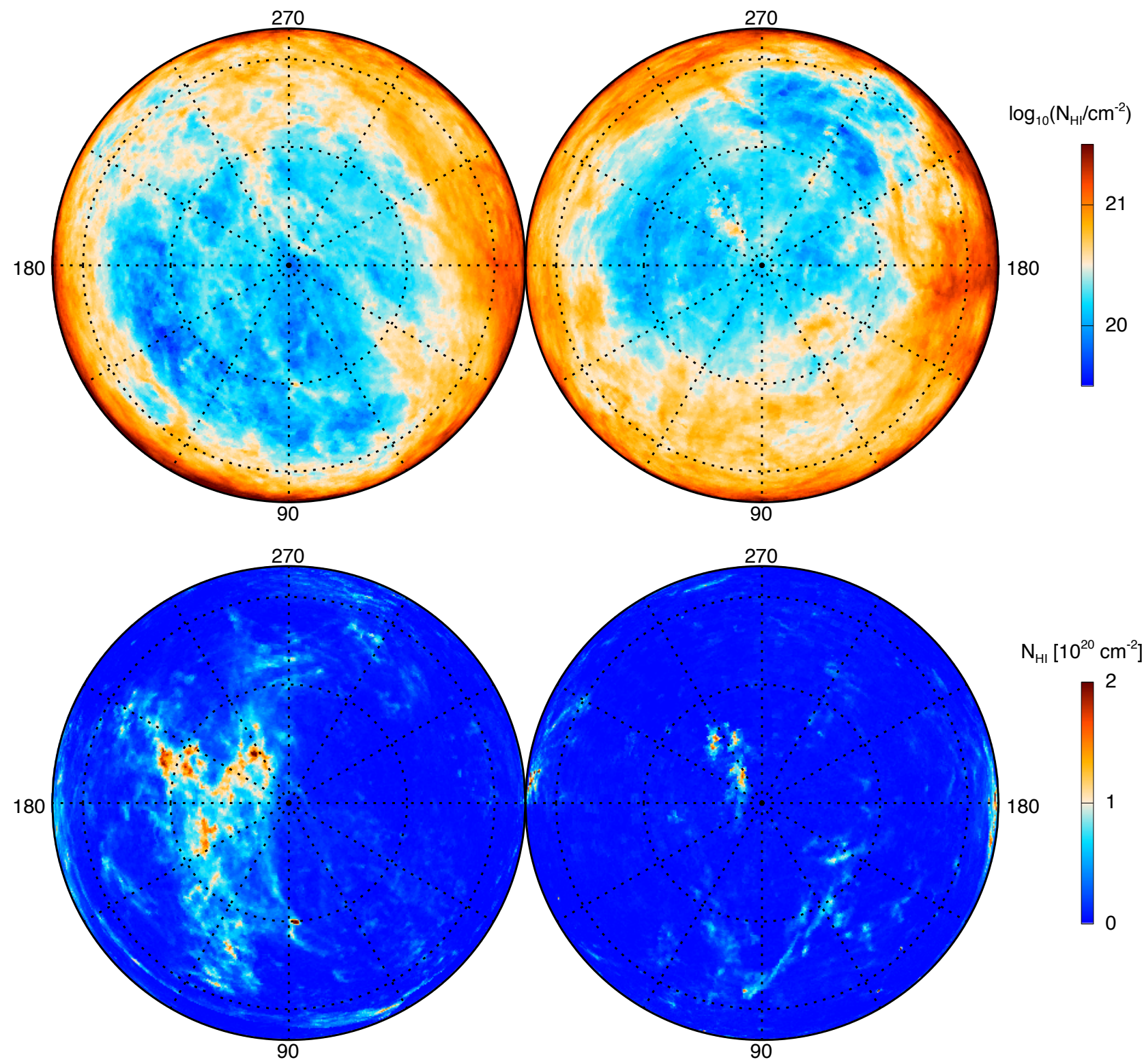

Fig. 2. Polar orthographic projections of the LVC map (upper) and the IVC map (lower) shown in Fig. 1. The left (right) panel is centred on the north (south) Galactic pole. Longitude increases clockwise (anticlockwise), with the two panels joining at $l=0^{\circ}$ and $b=0^{\circ}$. Dotted lines representing constant longitude and latitude are spaced by $30^{\circ}$. The radius from the pole is $\propto c \cos (b)$, so this projection emphasizes features at high latitude.

In the optically thin limit, the SED of emission from a uniform population of grains is well described, empirically, by a modified blackbody (MBB):

$I_{v}=\tau_{v} B_{v}(T)$,

where $I_{\nu}$ is the specific intensity, $B_{v}(T)$ is the Planck function for dust at temperature $T$, and $\tau_{v}$ is the frequency-dependent dust optical depth modifying the blackbody shape of the SED. The optical depth is the product of the dust opacity, $\sigma_{\mathrm{e} v}$, and the gas column density, $N_{\mathrm{H}}$ :

$\tau_{v}=\sigma_{\mathrm{e} v} N_{\mathrm{H}}$.

Alternatively, the optical depth is the product of the dust emissivity cross section per unit mass, $\kappa_{v}$ (in $\mathrm{cm}^{2} \mathrm{~g}^{-1}$ ), and the dust mass column density, $M_{\text {dust }}$ :

$\tau_{v}=\kappa_{v} M_{\text {dust }}$, where $M_{\text {dust }}=r \mu m_{\mathrm{H}} N_{\mathrm{H}}$, with $r$ being the dust-to-gas mass ratio, $\mu$ the mean molecular weight, and $m_{\mathrm{H}}$ the mass of a hydrogen atom. Note that $\kappa_{v}$ depends on the chemical composition and structure of dust grains, but not the size for particles small compared to the wavelength, as here. It is usually described as a power law $\kappa_{v}=\kappa_{0}\left(v / v_{0}\right)^{\beta}$ (Hildebrand 1983; Compiègne et al. 2011), where $\kappa_{0}$ is the emission cross-section at a reference frequency $v_{0}$. Put together, the emission of dust of a given composition and structure and in thermal equilibrium is:

$I_{v}=\kappa_{0}\left(v / v_{0}\right)^{\beta} r \mu m_{\mathrm{H}} N_{\mathrm{H}} B_{v}(T)$.

In practice the shape of the observed SED depends on three main parameters. First, the equilibrium temperature is set by the radiation field strength, parametrized by the scaling factor, $U$, of the mean interstellar radiation field (ISRF) in the solar neighbourhood from Mathis et al. (1983); note that in dense regions, $U$ is decreased because of attenuation. Second, the grain size 

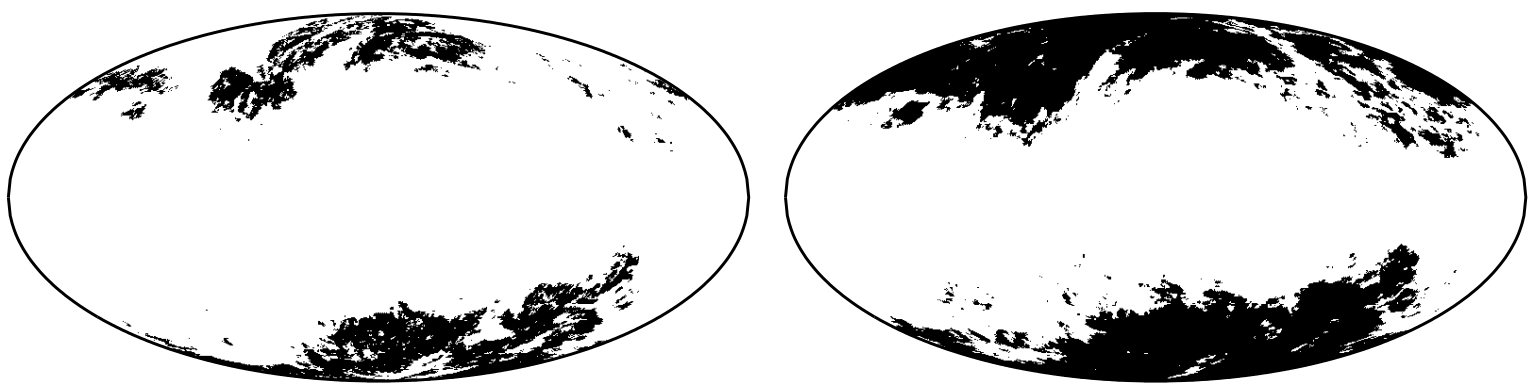

Fig. 3. Masks used to estimate the zero levels of the maps. Left: "low $N_{\mathrm{HI}}$ mask" including pixels of the sky where the LVC column density is $<2 \times 10^{20} \mathrm{~cm}^{-2}$ and the IVC column density is below $<0.1 \times 10^{20} \mathrm{~cm}^{-2}$. Right: mask where the total H I column density (LVC plus IVC) is lower than $3 \times 10^{20} \mathrm{~cm}^{-2}$.

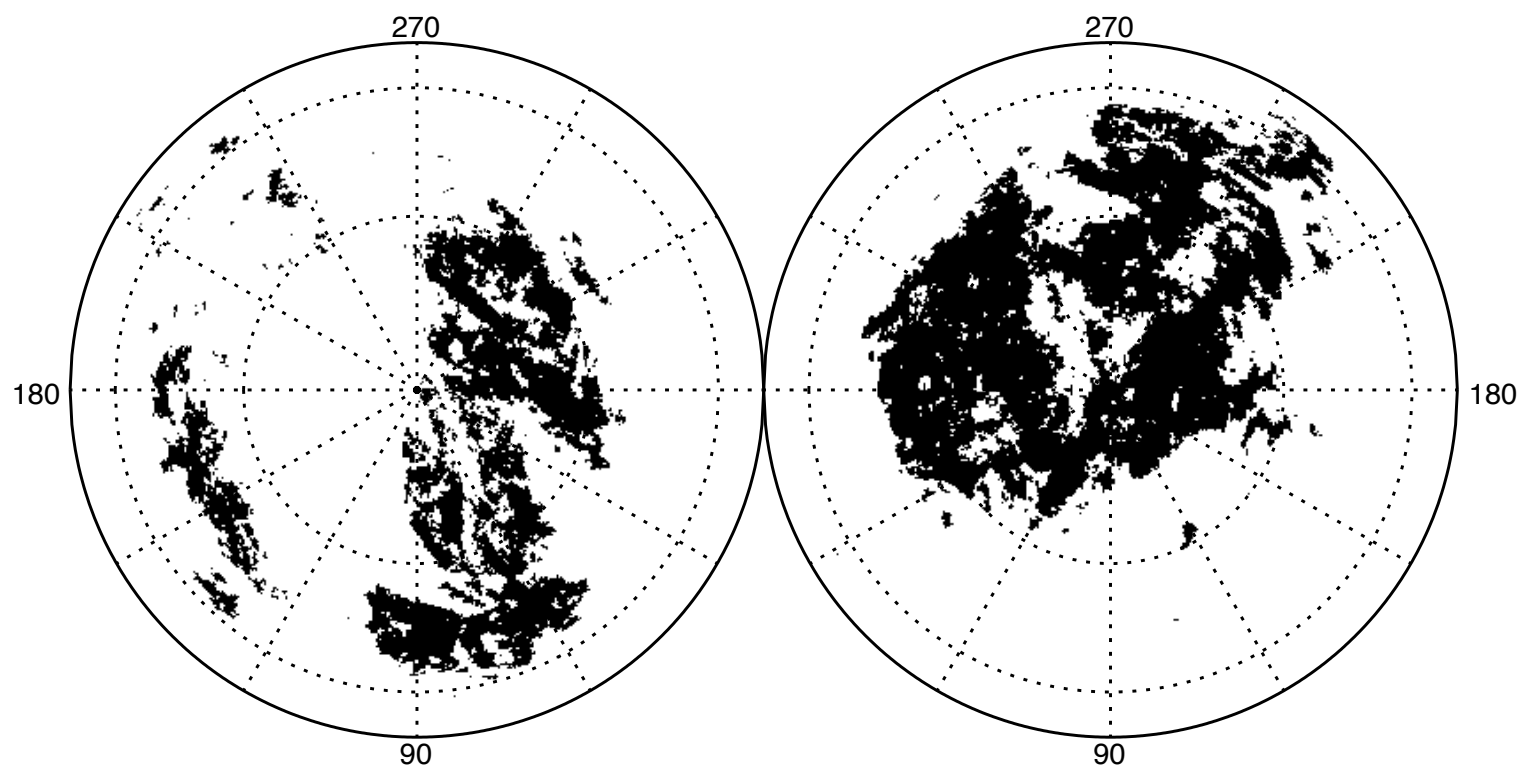

Fig. 4. Polar orthographic projection of the low $N_{\mathrm{HI}}$ mask shown in Fig. 3 left.
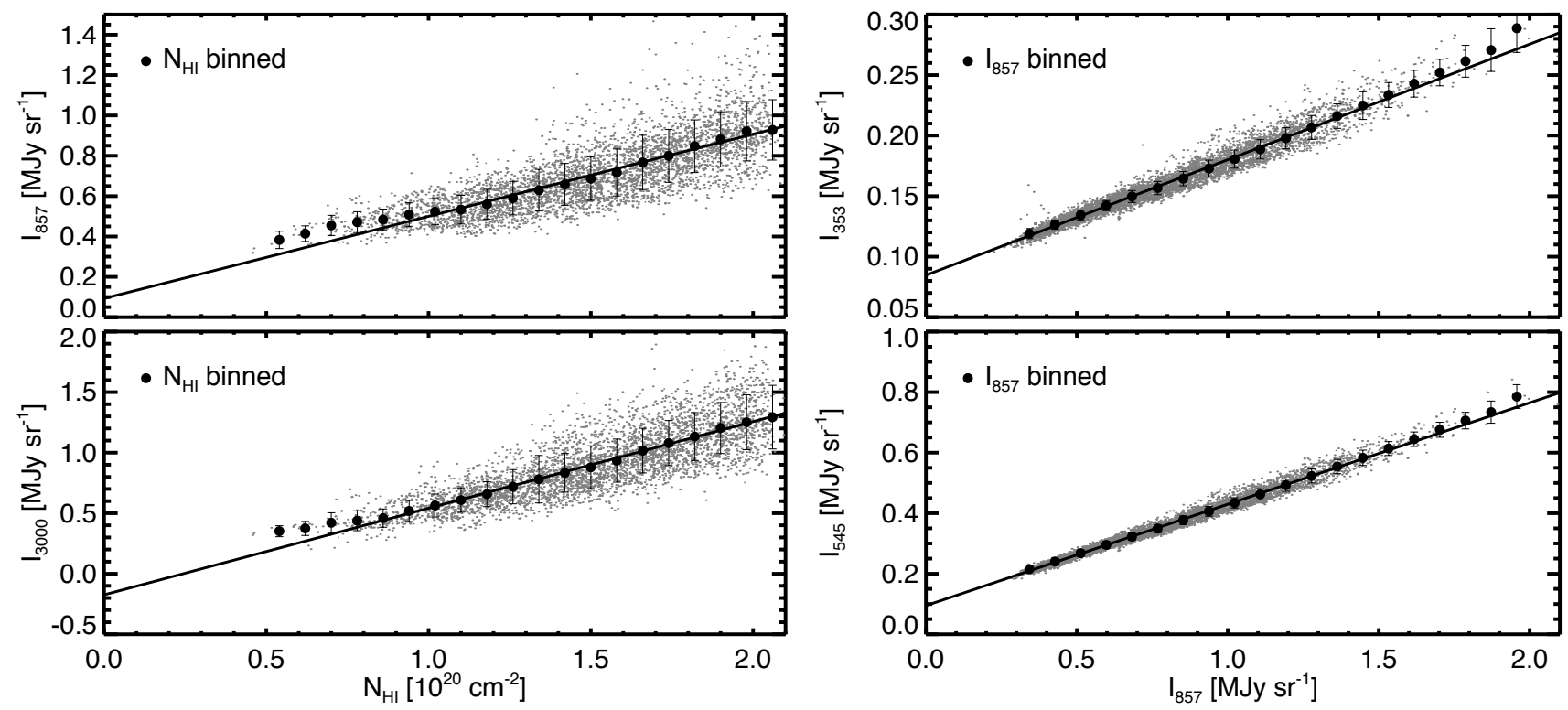

Fig. 5. Correlation plots used to estimate the Galactic zero levels of the IRAS and Planck maps (ZE subtracted). Left: correlation of 857 and $3000 \mathrm{GHz}$ vs. H I column density obtained on the $N_{\mathrm{HI}}<2 \times 10^{20} \mathrm{~cm}^{-2}$ mask (Fig. 3 bottom left). Right: correlation of 353 and $545 \mathrm{GHz}$ vs. $857 \mathrm{GHz}$ obtained on the $N_{\mathrm{HI}}<3 \times 10^{20} \mathrm{~cm}^{-2}$ mask (Fig. 3 bottom right). All maps in the analysis were smoothed to a common resolution of $1^{\circ}$. The black circles and the associated bars are the average and standard deviation of $I_{v}$ in bins of $N_{\mathrm{HI}}(l e f t)$ and $I_{857}(r i g h t)$. 
distribution (Mathis et al. 1977; Weingartner \& Draine 2001) is important; exposed to the same ISRF, bigger grains have a lower equilibrium temperature than smaller ones. Third, the dust structure and composition determine not only the optical and UV absorption cross section, but also the emission cross-section, the frequency-dependent efficiency to emit radiation, usually modelled as above as a power law $\left(\kappa_{0} \nu^{\beta}\right)$ but possibly more complex depending on dust properties $(\beta$ could vary with frequency and/or grain size and/or grain temperature). In a given volume element along the line of sight, the distribution of dust grain sizes will naturally create a distribution of equilibrium temperatures. In addition, dust properties might vary along the line of sight. Furthermore, $U$ might also change along some lines of sight. Therefore, the observed dust SED is a mixture of emission modified by these effects, the sum of several different MBBs. Nevertheless, the simplification of fitting a single MBB is often adopted and indeed here, with only four photometric bands available, is unavoidable. The parametrization of the MBB for the empirical fit is:

$I_{v}=\tau_{v_{0}} B_{v}\left(T_{\mathrm{obs}}\right)\left(\frac{v}{v_{0}}\right)^{\beta_{\mathrm{obs}}}$,

where $v_{0}$ is a reference frequency at which the optical depth $\tau_{v_{0}}$ is estimated ( $v_{0}=353 \mathrm{GHz}$ in our SED applications in this paper).

The main challenge is then to relate the parameters of the fit to physical quantities. It has been shown by many authors (Blain et al. 2003; Schnee et al. 2007; Shetty et al. 2009b; Kelly et al. 2012; Juvela \& Ysard 2012a,b; Ysard et al. 2012) that, in general, the values of $T_{\mathrm{obs}}$ and $\beta_{\mathrm{obs}}$ recovered from an MBB fit cannot be related simply to the mass-weighted average along the line of sight of the dust temperature and spectral index. Even for dust with a spectral index constant in frequency (i.e., $\beta$ does not depend on $v$ ), the distribution of grain sizes and the variations of $U$ along the line of sight could introduce a broadening of the SED relative to the case of a single dust size and single $U$. In addition, the dust luminosity is proportional to $T^{4+\beta}$ and so dust that is hotter for any reason, including efficiency of absorption, will contribute more to the emission at all frequencies than colder dust. Therefore, the observed SED is not a quantity weighted by mass alone. The dust SED is wider than a single MBB due to the distribution of $T$, and so the fit is bound to find a solution where $\beta_{\text {obs }}<\beta$ and, in consequence, where $T_{\text {obs }}$ is biased toward higher values. This results in dust optical depth that is generally underestimated: $\tau_{\text {obs }}<\tau$. This effect is somewhat mitigated when lower frequency data are included. In the Rayleigh-Jeans limit the effect of temperature is low and the shape of the spectrum is dominated by the true $\beta$. For $T=15-25 \mathrm{~K}$ dust, this range is at frequencies lower than $v=k T / h=310-520 \mathrm{GHz}$.

Models like the ones of Draine \& Li (2007) and Compiègne et al. (2011) go beyond the simple MBB parametrization by incorporating the variation of the equilibrium temperature of grains due to the size distribution. The model of Draine \& $\mathrm{Li}$ (2007) also includes a prescription for the variation of $U$ along the line of sight, but assumes fixed dust properties. Nevertheless, there are still many uncertainties in the properties of dust (the exact size distribution of big grains, the optical properties, and the structure of grains), in the evolution of these properties from diffuse to denser clouds, and in the variation of the radiation field strength along the line of sight.

Therefore, for our early exploration of the dust SED over the whole sky, at $5^{\prime}$ resolution and down to $353 \mathrm{GHz}(850 \mu \mathrm{m})$, we believe that it is useful to fit the dust SED using the empirical MBB approach, before attempting to use more physical models that rest on specific hypotheses. The three parameters $\tau_{v_{0}}, T_{\text {obs }}$ and $\beta_{\text {obs }}$ obtained from the MBB fit should be regarded as a way to fit the data empirically; the complex relationship between these recovered parameters and physical quantities needs to be investigated in detail with dedicated simulations (e.g., Ysard et al. 2012), but is beyond the scope of this paper.

\subsection{Implementation of the SED fit}

The fit of the dust SED with a MBB model has been carried out traditionally using a $\chi^{2}$ minimization approach. Recently, alternative methods for fitting observational data with limited spectral coverage have been proposed, based on Bayesian or hierarchical models (Veneziani et al. 2010, 2013; Kelly et al. 2012; Juvela et al. 2013). These new methods were developed specifically to limit the impact of instrumental noise on the estimated parameters. Even though these methods offer interesting avenues, we developed our own strategy to fit the dust SED over the whole sky because of another challenge to be mitigated, arising from the cosmic infrared background anisotropies (the CIBA). Although this has been overlooked, it can be dominant in the faint diffuse areas of the sky, as we demonstrate. We proceeded with a method based on the standard $\chi^{2}$ minimization (see Appendix B) but implemented a two-step approach that limits the fluctuations of the estimated parameters at small angular scales induced by noise and the CIBA. In developing the methodology we have explored using data degraded to lower resolution and smaller $N_{\text {side }}$.

\subsubsection{Frequency coverage}

One possible source of bias in the fit is the number of bands and their central frequency. The combination of Planck 353 to $857 \mathrm{GHz}$ and IRIS $3000 \mathrm{GHz}$ data allows us to sample the low and high frequency sides of the dust SED. For a typical temperature of $20 \mathrm{~K}$, the peak of the emission is at a frequency of $2070 \mathrm{GHz}$. This falls in a gap in the frequency coverage, between $857 \mathrm{GHz}$ and $3000 \mathrm{GHz}$. It is thus a concern that a fit of the Planck and IRIS data might bias the recovered parameters $T_{\text {obs }}$ and $\beta_{\text {obs }}$. To explore this we combined the Planck data with the DIRBE data at 1250,2143 , and $3000 \mathrm{GHz}(100,140$, and $240 \mu \mathrm{m}$ ), all smoothed to $60^{\prime}$, providing a better sample of the dust SED near its peak. We found that the recovered dust parameters are stable whether DIRBE data are used or not; no bias is observed in $T_{\text {obs }}, \beta_{\text {obs }}$, and $\tau_{353}$ compared with results obtained using just Planck and IRIS data.

We also evaluated the potential advantage of fitting the SED with only the Planck 353 to $857 \mathrm{GHz}$ data, a more coherent dataset not relying on the IRIS data. However, because the Wien part of the SED is not sampled the results showed a clear bias of $T_{\mathrm{obs}}$, toward lower values; consequently, when extrapolated to $100 \mu \mathrm{m}$, the fits greatly underestimate the emission detected in the IRIS data. Therefore, in the following the $\chi^{2}$ minimization fit was carried out on the data described in Sect. 2: the 857, 545, and $353 \mathrm{GHz}$ Planck maps, corrected for zodiacal emission, and the new $100 \mu \mathrm{m}$ map obtained by combining the IRIS and SFD maps.

\subsubsection{Noise and cosmic infrared background anisotropies}

Degeneracy (anticorrelation) of the estimated $T_{\text {obs }}$ and $\beta_{\text {obs }}$, inherent to the MBB fit of dust emission in the presence of noise, has had dedicated specific study (Shetty et al. 2009a; Juvela \& Ysard 2012a). As mentioned above, the CIBA is also a contaminating source in the estimate of the MBB parameters. 

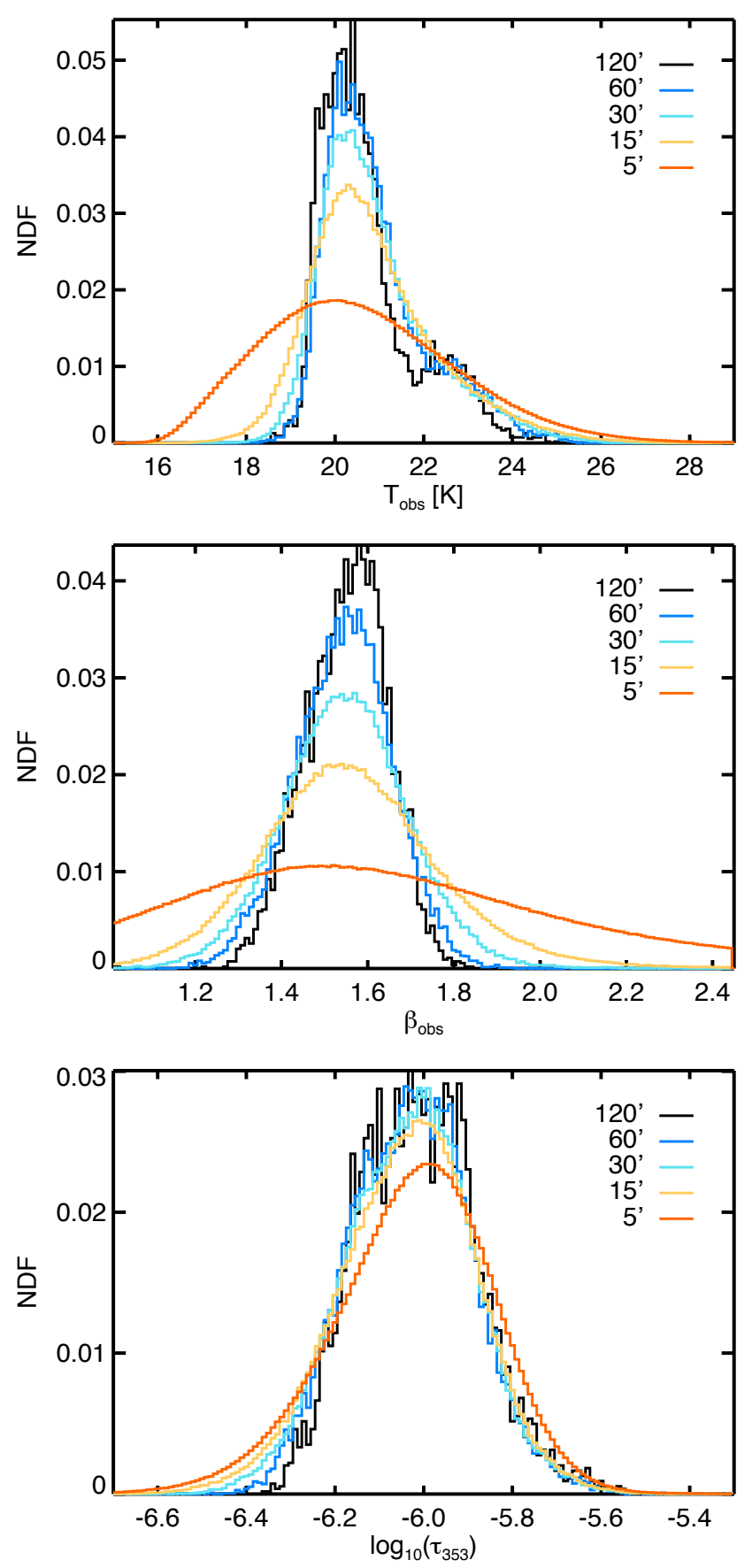

Fig. 6. Normalized distribution function (NDF) of $T_{\mathrm{obs}}$ (upper), $\beta_{\mathrm{obs}}$ (middle), and $\tau_{353}$ (lower) for data smoothed to different resolutions, from $5^{\prime}$ to $2^{\circ}$. The NDFs are shown only for the pixels corresponding to the low $N_{\mathrm{HI}}$ mask of Fig. 3, left, i.e., to the faintest $10 \%$ of the sky pixels.

The CIBA is the result of the combined emission of distant unresolved galaxies. Its structure on the sky is diffuse. The angular power spectrum, with $C_{\ell} \propto \ell^{-1}$ for $100<\ell<2000$ according to Planck Collaboration XVIII (2011) and Planck Collaboration XXX (2014), reveals the large-scale structure of the Universe at high redshift. The zero levels of the maps were set through correlation with $\mathrm{H} \mathrm{I}$ and so the data used in our study are insensitive to the monopole of the CIBA. However, the anisotropies, or fluctuations, are present in the maps. Because the CIBA power spectrum is flatter than that of interstellar dust emission $\left(C_{\ell} \propto \ell^{-2.9}\right.$,
Miville-Deschênes et al. 2007), in relative terms the CIBA is more visible at small scales. Another feature of the CIBA is that its structure on the sky is correlated in frequency, though only partially because galaxies at different redshifts contribute to the emission at different frequencies. Because of this partial correlation in frequency, the CIBA cannot be treated in the same way as instrumental noise in the fit. But it cannot be included as another component in the fitting function either. Nevertheless, the CIBA has an impact on the parameters of the fit; like the instrumental noise, the CIBA introduces an anticorrelation between $T_{\text {obs }}$ and $\beta_{\text {obs }}$.

One option to limit the effect of noise and the CIBA is to reduce the number of free parameters in the fit. In that context we have examined the possibility of fitting the dust SED over the whole sky, at $5^{\prime}$ resolution, using a fixed $\beta_{\text {obs }}$ with values between 1.5 and 1.8. A value of $\beta_{\text {obs }}=1.65$ provides the best fit with a reduced $\chi^{2}$ lower than unity everywhere on the sky but this is mostly due to the fact that we took into account calibration uncertainties in the fit (Appendix B). What is statistically significant is the fact that on about $25 \%$ of the sky the reduced $\chi^{2}$ is improved by letting $\beta_{\text {obs }}$ be a free parameter. This happens mostly in bright regions of the sky where the noise is not an issue. In molecular clouds and in the Galactic plane, there are variations in the shape of the SED that cannot be fit with only two parameters.

While fixing a parameter of the fit over the whole sky might be too strict, it might not be necessary to have all three parameters at full resolution to describe the data. We have thus evaluated the possibility of estimating one of the parameters at a lower resolution than the others. In the following we explore the impact of noise and the CIBA on the parameters of the fit as a function of angular resolution.

Both the noise and the CIBA have flatter power spectra than dust emission, and so we expect the intrinsic dust parameters $T_{\text {obs }}, \beta_{\text {obs }}$, and $\tau_{353}$ to have a smoother structure on the sky than noise and the CIBA, except perhaps in bright photon-dominated regions where the shape of the dust SED might vary rapidly at small scales due to radiative transfer effects and potentially fast dust evolution. Smoothing the maps by different amounts before fitting on each pixel therefore offers the advantage of revealing both this spatially smoother solution and the important impact of noise and the CIBA on the result of the fit. This is illustrated in Fig. 6 where we present normalized distribution functions (NDFs) of $T_{\mathrm{obs}}, \beta_{\mathrm{obs}}$, and $\tau_{353}$ obtained with data smoothed to $5^{\prime}, 15^{\prime}, 30^{\prime}, 60^{\prime}$, and $120^{\prime}$, selecting only pixels corresponding to the low $N_{\mathrm{HI}}$ mask (Fig. 3) to highlight a regime of relatively low signal-to-noise ratio. Smoothing the data has no real impact on the average value of the parameters, but the standard deviations of $T_{\text {obs }}$ and $\beta_{\text {obs }}$ go down rapidly with smoothing; at $5^{\prime}$ resolution the standard deviation of $T_{\mathrm{obs}}$ and $\beta_{\mathrm{obs}}$ is about twice as large as with smoothed data. On the other hand, the dispersion of $\tau_{353}$ is less affected by smoothing; it is dominated instead by cosmic variance, the considerable range of column densities even within this low $N_{\mathrm{H} \text { I }}$ mask $^{6}$. Of course the dust parameters might also vary at small scales and so a trade-off needs to be found.

To explore and quantify the impact of noise and the CIBA on the fit at different angular resolutions, for later comparison with the actual dispersion, we used Monte Carlo simulations of the

6 The dispersions of quantities normalized by the column density, $\sigma_{\mathrm{e} 353}=\tau_{353} / N_{\mathrm{H}}$ and the dust specific luminosity $L_{\mathrm{H}}$, are available only for lower resolutions; at $30^{\prime}$ resolution for this mask (see Table 4 in Sect. 4 below), they are considerably lower in fractional terms than the dispersion of $\tau_{353}$ in Fig. 6. 
Table 2. Results of Monte Carlo simulations of three-parameter fits: $1 \sigma$ uncertainties of $T_{\mathrm{obs}}$ and $\beta_{\mathrm{obs}}$ due to noise and the CIBA, for data at different resolutions.

\begin{tabular}{ccccc}
\hline \hline $\begin{array}{c}\theta \\
\text { arcmin }]\end{array}$ & $\begin{array}{c}\delta_{\text {noise }}\left(T_{\text {obs }}\right) \\
{[\mathrm{K}]}\end{array}$ & $\begin{array}{c}\delta_{\mathrm{CIBA}}\left(T_{\text {obs }}\right) \\
{[\mathrm{K}]}\end{array}$ & $\delta_{\text {noise }}\left(\beta_{\text {obs }}\right)$ & $\delta_{\mathrm{CIBA}}\left(\beta_{\text {obs }}\right)$ \\
\hline 5 & 2.1 & 0.39 & 0.49 & 0.11 \\
15 & 0.32 & 0.32 & 0.054 & 0.070 \\
30 & 0.15 & 0.23 & 0.026 & 0.049 \\
60 & 0.075 & 0.16 & 0.013 & 0.035 \\
120 & 0.037 & 0.11 & 0.0064 & 0.024 \\
\hline
\end{tabular}

Notes. The simulation was done for a single dust SED typical of the $10 \%$ faintest area of the sky, whose parameters are the median values found in the low $N_{\mathrm{HI}}$ mask: $T_{\mathrm{obs}}=20.8 \mathrm{~K}, \beta_{\mathrm{obs}}=1.55$ and $\tau_{353}=9.6 \times$ $10^{-7}$ (Table 3 ). The CIBA was modelled assuming partial correlation in frequency (see Appendix $\mathrm{C}$ for details). The noise and CIBA levels used for each resolution are given in Table C.1. The values given here are the standard deviations of the parameters $T_{\mathrm{obs}}$ and $\beta_{\mathrm{obs}}$ obtained from three-parameter SED fits of $10^{5}$ realizations; $\delta_{\text {noise }}$ and $\delta_{\mathrm{CIBA}}$ represent the separate contributions of noise and the CIBA to the total standard deviation.

SED, including dust emission, noise, and the CIBA. The details of the Monte Carlo simulations, including the information on the inter-frequency coherence, are described in Appendix C. We considered five different angular resolutions of the data: $5^{\prime}, 15^{\prime}$, $30^{\prime}, 60^{\prime}$, and $120^{\prime}$. The noise and CIBA levels used for each resolution are given in Table C.1. Here we present results for an SED appropriate to Fig. 6 by adopting the median dust parameters found in the low $N_{\mathrm{HI}}$ mask that corresponds to the faintest $10 \%$ of the sky. We simulated $10^{5}$ realizations of this SED to which noise and the CIBA were added. For each realization the three parameters $\tau_{353}, T_{\mathrm{obs}}$, and $\beta_{\mathrm{obs}}$ were estimated as in Appendix B. The $1 \sigma$ dispersions of $T_{\mathrm{obs}}$, and $\beta_{\mathrm{obs}}$ obtained at each resolution are given in Table 2, for noise and the CIBA separately. The simulated effect of smoothing on the $\beta_{\mathrm{obs}}-T_{\mathrm{obs}}$ anticorrelation is shown in Fig. 7.

At full resolution the noise is the dominant source of error on the retrieved parameters. For the specific faint dust spectrum considered here, the noise produces an uncertainty of $2.1 \mathrm{~K}$ while the uncertainty due to the CIBA is only $0.39 \mathrm{~K}$. The same is true for $\beta_{\text {obs }}$ : the uncertainties are 0.49 and 0.11 for the noise and the CIBA, respectively. However, even with moderate smoothing of the data, the impact of noise on the fit reduces sharply, whereas the reduction of the impact of the CIBA is less dramatic. This arises because the CIBA has a power spectrum that is steeper than that of typical (white) noise. In addition, unlike the CIBA, noise has power up to the pixel scale (i.e., it is not attenuated by the beam). For example, as seen in Table 2, for data smoothed to $30^{\prime}$, the noise levels of the Planck and IRAS data go down by a factor 18.5 while the CIBA standard deviation decreases only by a factor 2.5 . As a result, our simulations show that for data smoothed to resolution larger than $15^{\prime}$ the CIBA becomes the main source of error.

Similar relative effects as a function of resolution are seen in the $\beta_{\text {obs }}-T_{\text {obs }}$ anticorrelation in Fig. 7. At all resolutions the estimates of $T_{\mathrm{obs}}$ and $\beta_{\mathrm{obs}}$ lie within an ellipse in $\beta_{\mathrm{obs}}-T_{\mathrm{obs}}$ space. The orientation and extent of the ellipse depends on the amplitudes of the noise and of the CIBA, which are both different and in a different ratio at each resolution. In all cases the ellipse is centred on the input values, demonstrating that noise and the CIBA do not bias the estimate of $T_{\text {obs }}$ and $\beta_{\text {obs }}$. This is the case even though the CIBA has a flatter (broader) SED than

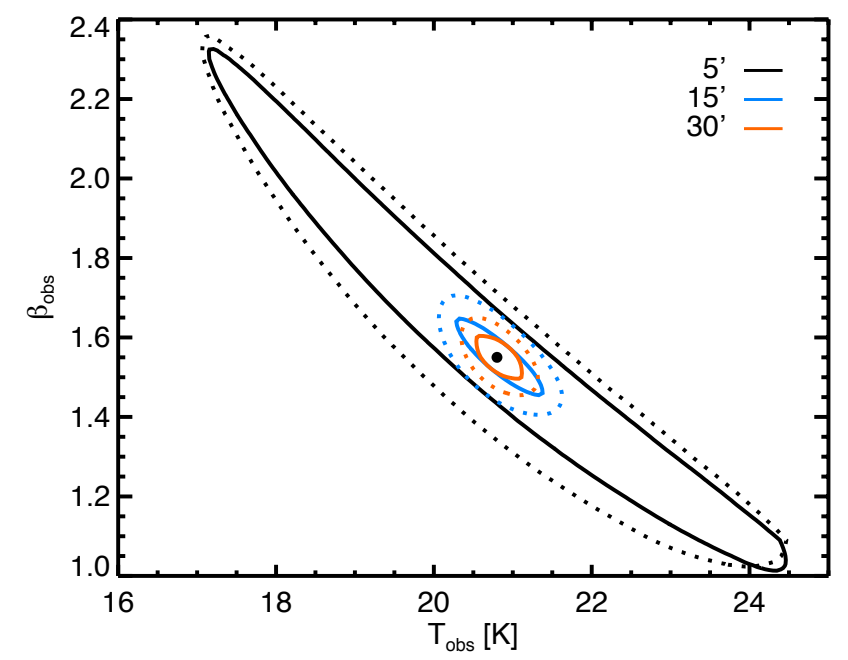

Fig. 7. $\beta_{\mathrm{obs}}-T_{\mathrm{obs}}$ diagram showing the $1 \sigma$ contour of the fit of a single SED (sum of dust emission, noise, and the CIBA) for simulated data with $\tau_{353}=9.6 \times 10^{-7}$ and noise and the CIBA levels for each resolution from Table C.1: $5^{\prime}$ (black), 15' (blue) and 30' (red). Contours are shown for noise only (solid) and for noise and the CIBA (dotted). The other two parameters of the dust emission used in this simulation, marked by a black dot, are, like $\tau_{353}$, the median values found in the low $N_{\mathrm{HI}}$ mask: $T_{\mathrm{obs}}=20.8 \mathrm{~K}$ and $\beta_{\mathrm{obs}}=1.55$.

interstellar dust. Because the CIB monopole was removed in the data and therefore not included in the simulation, the CIBA produces as many negative as positive CIB fluctuations on the sky at each frequency. Because they are (partially) correlated in frequency, positive CIB fluctuations bias the SED and descriptive dust parameters toward a flatter SED while negative CIB fluctuations have the opposite effect, toward a steeper SED.

\subsubsection{The two-step approach}

Given the impact of noise and the CIBA on the recovered parameters, described in the previous section, we have chosen to fit the data in two steps. First, we fit the data smoothed to $30^{\prime}$ (but on the $N_{\text {side }}=2048$ grid). As shown in Figs. 6 and 7 this greatly reduces the effect of noise on the estimate of $\beta_{\mathrm{obs}}$ and $T_{\mathrm{obs}}$. Second, we fit the data at $5^{\prime}$ resolution with a fixed $\beta_{\text {obs }}$ taken from the map of $\beta_{\text {obs }}$ obtained with data at $30^{\prime}$ resolution. That way two degrees of freedom $\left(\tau_{353}\right.$ and $T_{\text {obs }}-$ see Eq. (6)) are still available to capture the variations of the dust SED at full resolution while limiting the effect of the $\beta-T$ degeneracy due to noise.

This two-step approach is in the same spirit as the one implemented in the Commander-Ruler algorithm (Planck Collaboration XII 2014). The advantage of such methods arises by favouring a spatially smoother solution for parameters that are not expected to vary strongly at small scale. In the second fit we chose to fix $\beta_{\text {obs }}$ rather than $T_{\text {obs }}$. It is not yet clear how the actual spectral index of the grain opacity, $\beta$, might vary on small scales (some models even assume that it is constant: Draine \& Li 2007; Compiègne et al. 2011). However, the dust temperature is expected to vary on small scales, especially in dense regions of the ISM due to the attenuation of the radiation field.

We performed Monte Carlo simulations to evaluate the contributions of noise and the CIBA (partly correlated in frequency - see Appendix C) to variations, whence uncertainties, of the recovered $T_{\mathrm{obs}}$ and $\beta_{\mathrm{obs}}$ for the specific case of the adopted two-step fit $\left(30^{\prime}\right.$ and $\left.5^{\prime}\right)$. Figure 8 illustrates the uncertainties of 

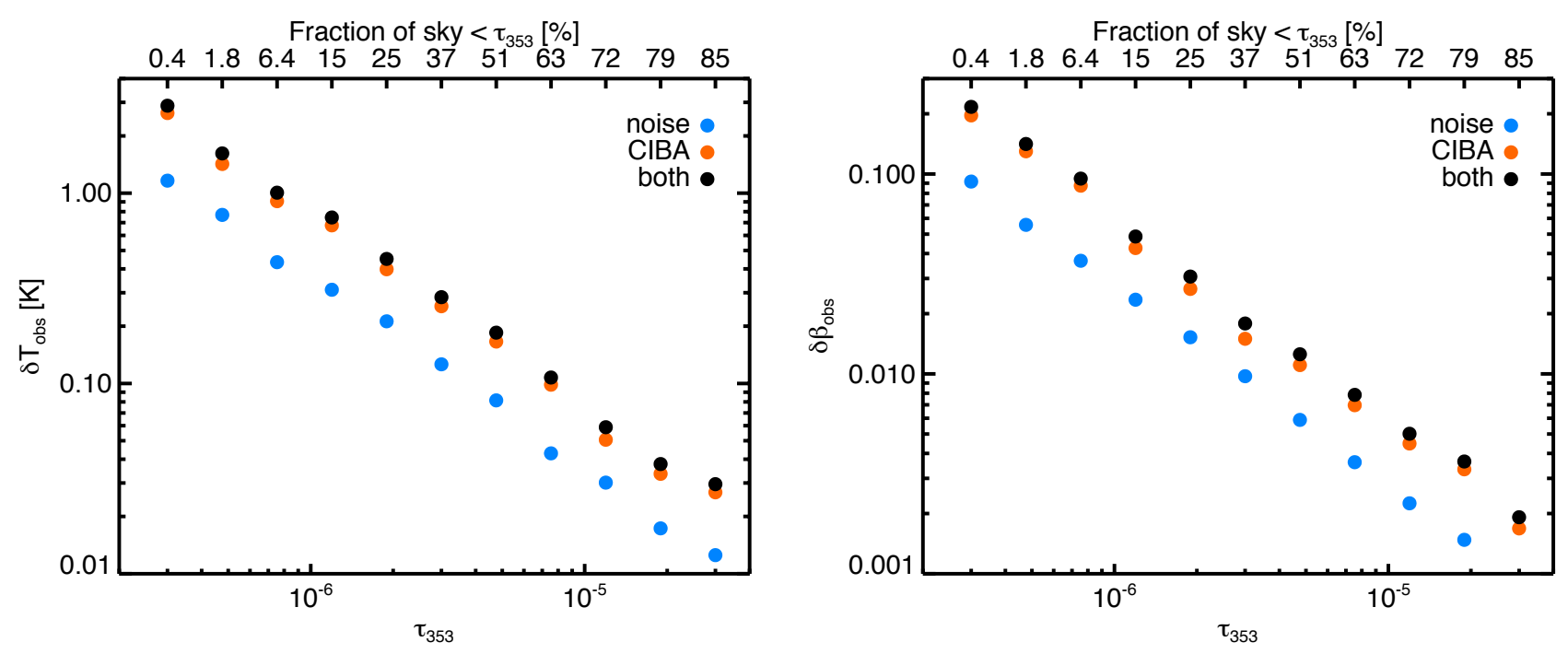

Fig. 8. Estimate of the uncertainties of $T_{\text {obs }}$ (left $)$ and $\beta_{\text {obs }}(r i g h t)$ due to noise and the CIBA for a two-step fit to single dust SEDs with $T_{\text {obs }}=20.8 \mathrm{~K}$ and $\beta_{\mathrm{obs}}=1.55$ but increasing $\tau_{353}$. These results were obtained using Monte Carlo simulations (see Appendix C). The fits for $\beta_{\mathrm{obs}}$ were carried out assuming noise and CIBA levels at $30^{\prime}$ and a free $T_{\text {obs. }}$. The fits for $T_{\text {obs }}$ were carried out assuming noise and CIBA levels at $5^{\prime}$ and a fixed $\beta_{\mathrm{obs}}=1.55$. The dots give the contribution of the noise (blue) and the CIBA (orange) to the uncertainties, and the quadratic sum of the two (black). The top axes of both plots indicate the fraction of sky that has $\tau_{353}$ lower than the value on the lower axis.

$T_{\text {obs }}$ and $\beta_{\text {obs }}$ arising from noise and the CIBA for dust SEDs on lines of sight with increasing $\tau_{353}$.

For the typical SED $\left(\tau_{353}=9.6 \times 10^{-7}, T_{\text {obs }}=20.8 \mathrm{~K}\right.$, and $\left.\beta_{\mathrm{obs}}=1.55\right)$ corresponding to the $10 \%$ faintest area of the sky, we made a comparison of the Monte-Carlo results for the twostep fit and the direct three-parameter fit (Sect. 3.2.2). The uncertainties of the direct fit at $5^{\prime}$ are $\delta\left(T_{\text {obs }}\right)=2.1 \mathrm{~K}$ and $\delta\left(\beta_{\text {obs }}\right)=$ 0.50 , adding the contributions of noise and CIBA in quadrature (see Table 2). For the same SED parameters, the uncertainties of the two-step fit are $\delta\left(T_{\text {obs }}\right)=0.8 \mathrm{~K}$ and $\delta\left(\beta_{\text {obs }}\right)=0.06$ (see Fig. 8). In addition, the results of the two-step fit simulations indicate that for both $T_{\mathrm{obs}}$ at $5^{\prime}$ and $\beta_{\mathrm{obs}}$ at $30^{\prime}$ the CIBA has a greater contribution than the noise, contrary to the situation for the direct three-parameter fit.

For the faintest $0.4 \%$ of the sky (top axis in Fig. 8), the results of the simulations indicate that the combined effects of noise and the CIBA produce variations $\delta T_{\mathrm{obs}}=3.0 \mathrm{~K}$ and $\delta \beta_{\mathrm{obs}}=0.2$. On the other hand, for about $93 \%$ of the sky the variations are much smaller, $\delta T_{\text {obs }}<1.0 \mathrm{~K}$ and $\delta \beta_{\text {obs }}<0.1$, i.e., $<5 \%$ and $<6 \%$ fractional error, respectively. This is in accordance with the fact that at $353 \mathrm{GHz}$, where the CIBA is the strongest contaminant, about $93 \%$ of the sky has $I_{353}>3 \sigma_{\mathrm{CIBA}}(353)$.

\subsection{Parameters and uncertainties}

The all-sky maps of the dust parameters, $T_{\text {obs }}, \beta_{\text {obs }}$, and $\tau_{353}$, and of their fractional uncertainties are presented in Figs. 9 and 10, respectively. The precision of the three parameters is of the order of a few percent on most of the sky. The uncertainties shown here are based on the statistical ones returned by the $\chi^{2}$ minimization fit assuming that the model is a good representation of the data. For $\beta_{\text {obs }}$ the uncertainty is from the $30^{\prime}$ fit. For $T_{\text {obs }}$ we added quadratically the fractional uncertainties from the $30^{\prime}$ and $5^{\prime}$ fits to include the covariance between $T_{\mathrm{obs}}$ and $\beta_{\mathrm{obs}}$, whence

$\delta T_{\mathrm{obs}}=T_{\mathrm{obs}} \sqrt{\left(\frac{\delta T_{\mathrm{obs}, 30}}{T_{\mathrm{obs}, 30}}\right)^{2}+\left(\frac{\delta T_{\mathrm{obs}, 5}}{T_{\mathrm{obs}, 5}}\right)^{2}}$, where the subscripts 5 and 30 refer to the parameter or uncertainty maps obtained at $5^{\prime}$ and $30^{\prime}$, respectively. Similarly, the uncertainty of $\tau_{353}$ is from the quadratic sum of the fractional uncertainties of $I_{353}^{\mathrm{m}}$ and $B_{353}\left(T_{\mathrm{obs}}\right)$ where $I_{353}^{\mathrm{m}}$ is the reconstructed model of the emission at $353 \mathrm{GHz}$. To estimate the uncertainty of $B_{353}\left(T_{\text {obs }}\right)$, we simply computed $\max \mid B_{353}\left(T_{\text {obs }} \pm \delta T_{\text {obs }}\right)-$ $B_{353}\left(T_{\text {obs }}\right)$.

The three uncertainty maps have a similar spatial structure. In general the fractional uncertainties are higher in the most diffuse areas of the sky (where the noise and the CIBA have a more important contribution) and in the inner Galaxy region. Striping patterns are visible, especially in the $T_{\text {obs }}$ uncertainty map; these are likely to be coming from the IRAS data. The uncertainty of $T_{\text {obs }}$ is of the order of $1-3 \%$ in bright areas, with a noticeable increase in the inner Galaxy and rising to $5-8 \%$ in the most diffuse areas of the sky. The same general trend is seen for $\tau_{353}$ but with higher values: $2-5 \%$ in bright areas and up to $10 \%$ in diffuse areas. The uncertainty of $\beta_{\mathrm{obs}}$, based on analysis at $30^{\prime}$ resolution, has a slightly different spatial structure. It is typically of $3-4 \%$ with a smaller decrease in bright areas and a noticeable increase in the inner Galaxy to $6-8 \%$.

The reduced $\chi^{2}$ of the fit is much smaller than unity over most of the sky, due to the fact that calibration uncertainties are taken into account in the fit to give less weight to data points with less precise calibration (Appendix B). To illustrate this, Fig. 11 shows the distribution function of (Data-Model)/Noise for each frequency used in the fit. The noise used here follows the definition of Eq. (B.1); it takes into account instrumental noise and the uncertainties of the calibration, the zero level, and the CMB subtraction. The range adopted in Fig. 11 corresponds to only $\pm 1 \sigma$. At 353 and $3000 \mathrm{GHz}$, for most of the sky pixels the data are fitted more tightly (to better than $0.1 \sigma$ ) than at 545 and $857 \mathrm{GHz}$. This implies that 353 and $3000 \mathrm{GHz}$ have a lot of weight in the estimation of the parameters. The $3000 \mathrm{GHz}$ band provides the only data point on the Wien part of the MBB and therefore strongly influences the determination of $T_{\mathrm{obs}}$. On the other hand, the $353 \mathrm{GHz}$ band strongly influences the determination of $\tau_{353}$ and $\beta_{\text {obs }}$ because it is the closest to the Rayleigh-Jeans part of the spectrum. The compensating small offsets of the distributions at 

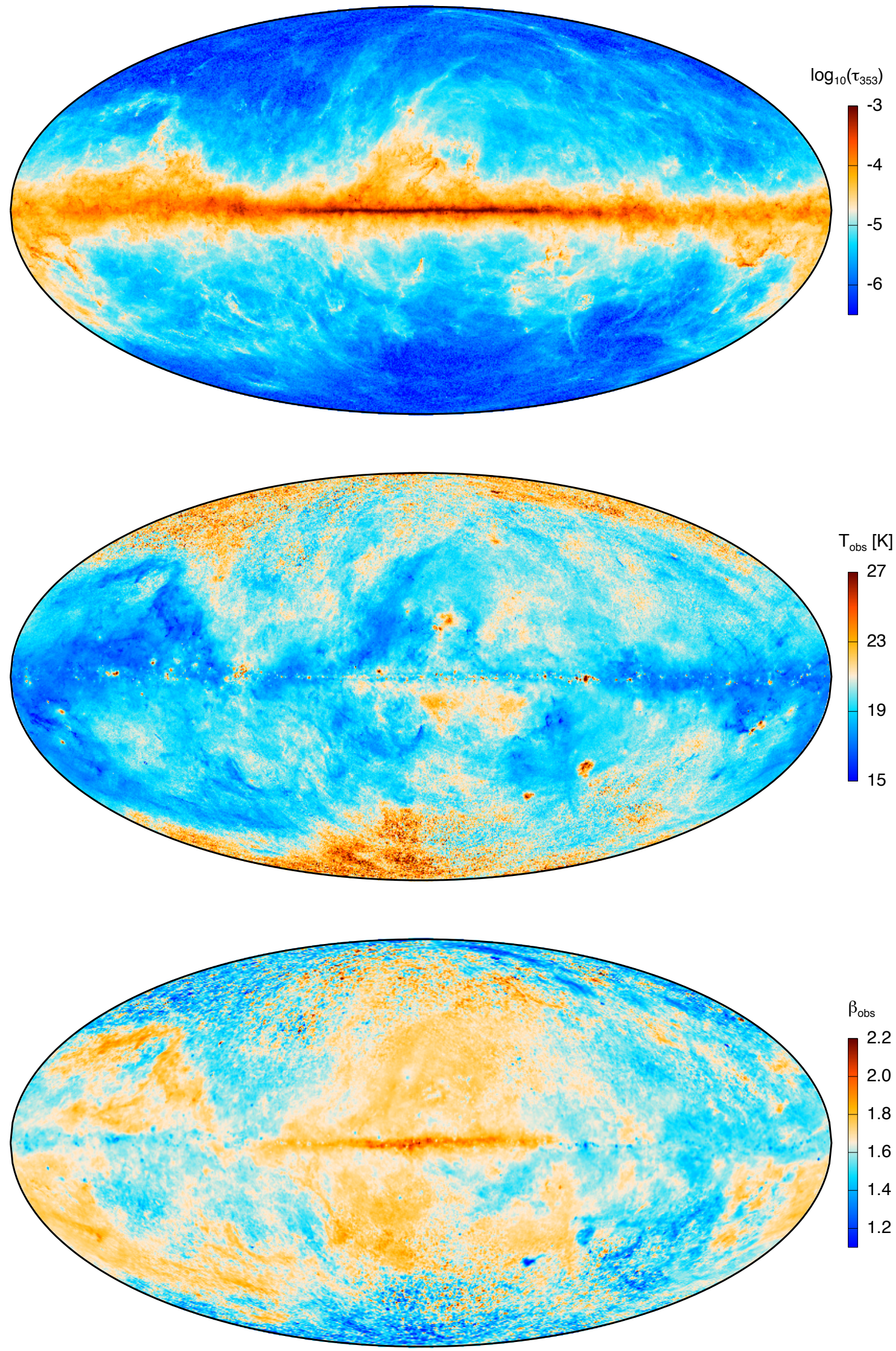

Fig. 9. All-sky maps of the parameters of the MBB fit of Planck 353, 545, and $857 \mathrm{GHz}$ and IRAS $100 \mu \mathrm{m}$ data. Upper: optical depth at $353 \mathrm{GHz}$, $\tau_{353}$, at $5^{\prime}$ resolution, displayed logarithmically (the range shown corresponds to $-6.5<\log _{10}\left(\tau_{353}\right)<-3$ ). Middle: observed dust temperature, $T_{\mathrm{obs}}$, at $5^{\prime}$ resolution, in kelvin. Lower: observed dust spectral index, $\beta_{\mathrm{obs}}$, at $30^{\prime}$ resolution. 

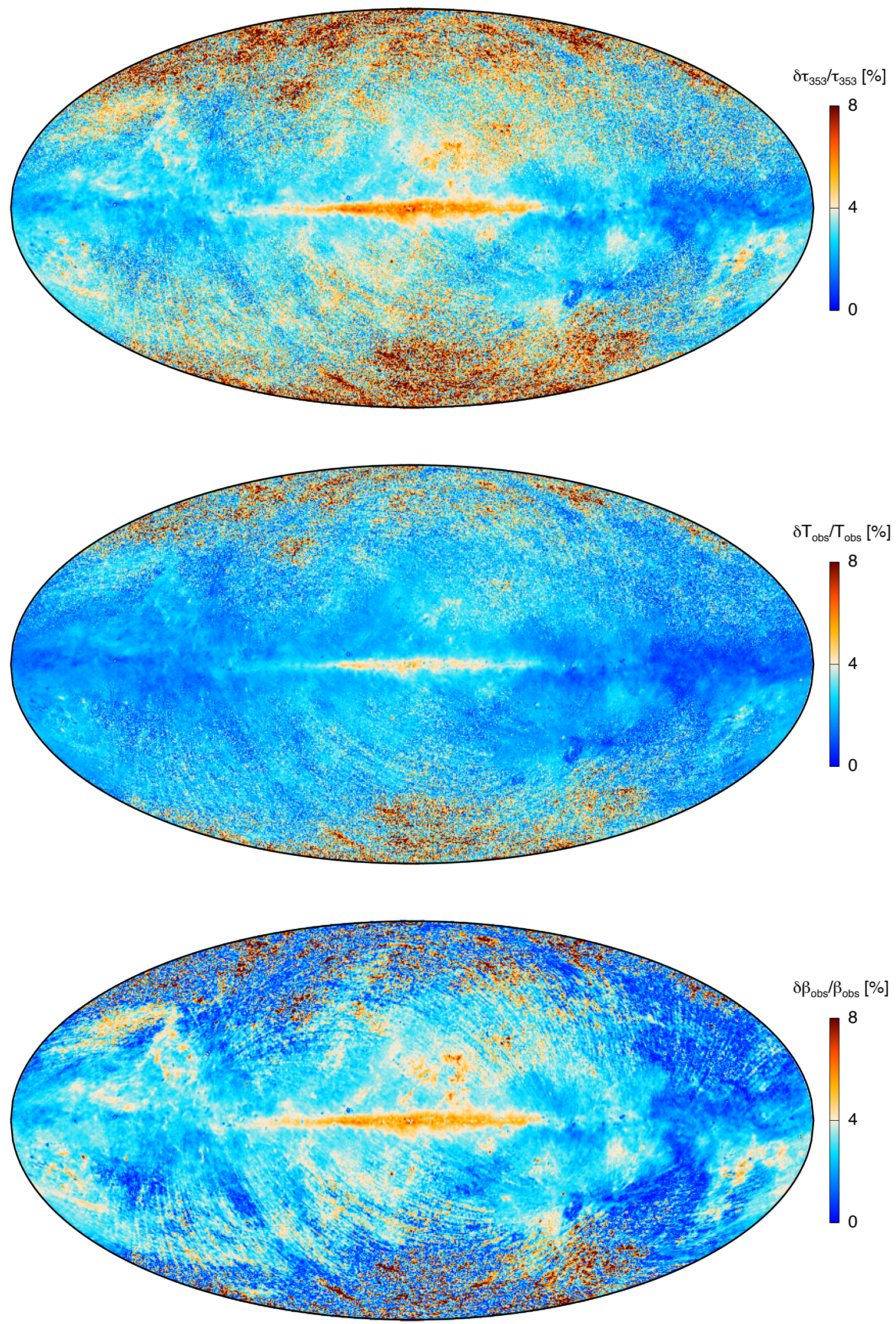

Fig. 10. All-sky maps of the fractional uncertainty (in percent) of the parameters of the MBB fit of Planck 353, 545, and 857 GHz and IRAS $100 \mu \mathrm{m}$ data. Upper: optical depth at $353 \mathrm{GHz}, \tau_{353}$, at $5^{\prime}$ resolution. Middle: observed dust temperature, $T_{\text {obs }}$, at $5^{\prime}$ resolution. Lower: observed dust spectral index, $\beta_{\mathrm{obs}}$, at $30^{\prime}$ resolution. 


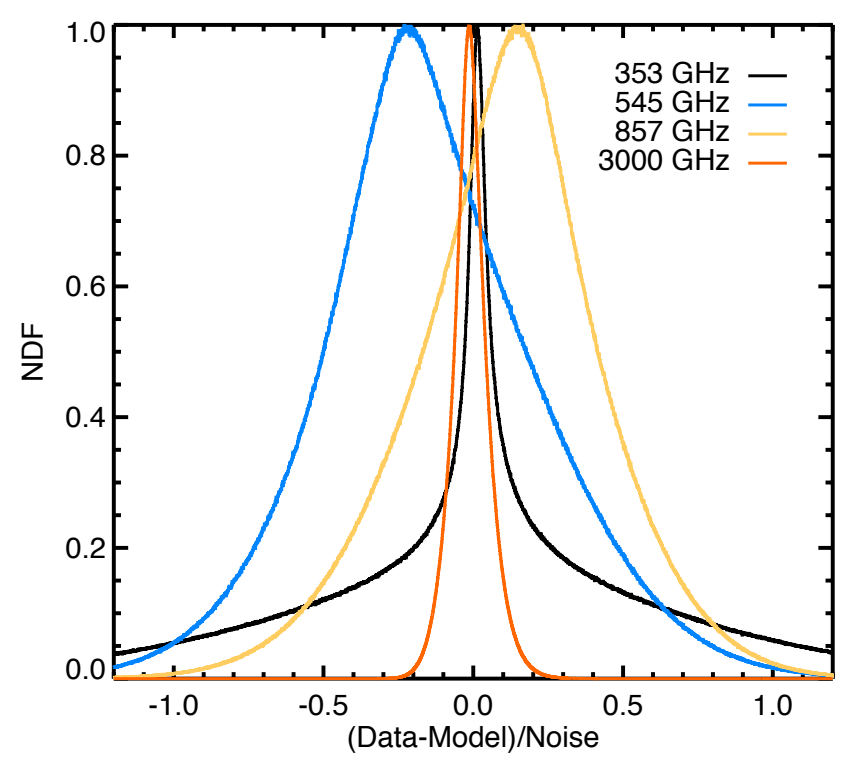

Fig. 11. Normalized distribution function of (Data - Model)/Noise for the four frequencies used in the fit.

the other two frequencies might suggest that the adopted model does not adequately describe the data. However, these offsets are well within the calibration uncertainties of the data; the overall reduced $\chi^{2}$ is lower than unity and these offsets might be removed by a small systematic change in the relative calibration of the data. Given the actual precision of the calibration, it would be premature to conclude that a more complex model is required to fit the data.

We compared the reduced $\chi^{2}$ with that from a fit of the data at $5^{\prime}$ with $\tau_{353}, T_{\text {obs }}$, and $\beta_{\text {obs }}$ as free parameters. We were looking for pixels on the sky for which the two-step fitting procedure provides a reduced $\chi^{2}$ greater than unity (i.e., a relatively bad fit) while fitting the three parameters simultaneously at full resolution would provide a better solution with a lower reduced $\chi^{2}$. This occurred for only $0.3 \%$ of the pixels. These pixels, possibly dominated by galaxies, are grouped in small-scale structures located at high Galactic latitude and away from bright interstellar areas.

\subsection{Dust radiance}

In the previous sections we have described the properties of the parameters that define the shape of the dust SED. Now we examine the dust radiance or dust integrated intensity defined as

$\mathcal{R}=\int_{v} I_{v} \mathrm{~d} v$

Because the grains are in thermal equilibrium, this also represents the energy absorbed. Here we estimate $\mathcal{R}$ at each sky position by integrating the MBB fit:

$\mathcal{R}=\int_{v} \tau_{353} B_{v}\left(T_{\mathrm{obs}}\right)\left(\frac{v}{353}\right)^{\beta_{\mathrm{obs}}} \mathrm{d} v$.

This can be done analytically in terms of the Gamma $(\Gamma)$ and Riemann zeta functions $(\zeta)$ :

$\mathcal{R}=\tau_{353} \frac{\sigma_{\mathrm{S}}}{\pi} T_{\mathrm{obs}}^{4}\left(\frac{k T_{\mathrm{obs}}}{h v_{0}}\right)^{\beta_{\mathrm{obs}}} \frac{\Gamma\left(4+\beta_{\mathrm{obs}}\right) \zeta\left(4+\beta_{\mathrm{obs}}\right)}{\Gamma(4) \zeta(4)}$, where $\sigma_{\mathrm{S}}$ is the Stefan-Boltzmann constant, $k$ is the Boltzmann constant, $h$ is the Planck constant, and $v_{0}=3.53 \times 10^{11} \mathrm{~Hz}$. Using the fit parameters described above we produced the all-sky map of $\mathcal{R}$ shown in Fig. 12, expressed in units of $\mathrm{W} \mathrm{m}^{-2} \mathrm{sr}^{-1}$.

Note that even though the calculation of $\mathcal{R}$ uses the dust parameters $\left(T_{\mathrm{obs}}, \beta_{\mathrm{obs}}, \tau_{353}\right), \mathcal{R}$ does not suffer from any degeneracy in the fit parameters. In this context the MBB should be seen as an interpolating function; $\mathcal{R}$ is not very sensitive to the assumptions made in fitting the SED as long as the fit accounts for the data, including the high-frequency turnover. The uncertainty of $\mathcal{R}$ arises mostly from the calibration uncertainty of the data and, to a lesser extent, from the limited number of bands used in the $\mathrm{fit}^{7}$.

In thermal equilibrium, $\mathcal{R}$ is equal to the amount of light absorbed by dust. Assuming constant properties along the line of sight, including the dust-to-gas ratio,

$\mathcal{R} \propto U \overline{\sigma_{\mathrm{a}}} N_{\mathrm{H}}$

where $\overline{\sigma_{\mathrm{a}}}$ is the absorption opacity defined similarly to the emission opacity in Eq. (3), averaged over the size distribution and also, in this case, over the spectrum of the ISRF.

This is complementary to $\tau_{353}$, which is also used as a proxy for $N_{\mathrm{H}}$ :

$\tau_{353}=\frac{I_{353}}{B_{353}\left(T_{\text {obs }}\right)}=\sigma_{\mathrm{e} 353} N_{\mathrm{H}}$.

Division by the Planck function factors out any effects due to spatial variations of the dust temperature (potentially linked to spatial variations of $U$ ), but $\tau_{353}$ is only proportional to $N_{\mathrm{H}}$ if the dust opacity $\sigma_{\mathrm{e} 353}$ is constant. This limitation does not apply to $\mathcal{R}$, which is independent of $\sigma_{\mathrm{e} 353}$ because of thermal equilibrium; $\mathcal{R}$ is simply the energy emitted by dust (Eq. (8)), whatever the shape of the SED and regardless of how efficient the grain cooling is. Thus $\mathcal{R}$ is closer to a measured quantity, while $\tau_{353}$ is a parameter deduced from a model.

At high Galactic latitudes, where the spatial variations of $U$ and $\sigma_{\mathrm{e} 353}$ are expected to be minimal so that both $\tau_{353}$ and $\mathcal{R}$ should be proportional to dust column density, comparison of maps of $\tau_{353}$ and $\mathcal{R}$ reveals another fundamental difference, as illustrated in Fig. 13 for one of the faintest areas in the sky: the map of $\tau_{353}$ shows surprisingly strong small-scale fluctuations that are absent in the map of $\mathcal{R}$.

This significant difference is due to the impact of the CIBA, especially its decorrelation in frequency. On the one hand, $\tau_{353}$ is the division of $I_{353}$ by $B_{353}\left(T_{\text {obs }}\right.$ ) (Eq. (12)) and so is contaminated by the CIBA at not only $353 \mathrm{GHz}$ but also $3000 \mathrm{GHz}$; i.e., because the $3000 \mathrm{GHz}$ band is the only one in the Wien range, it has a strong weight in the determination of $T_{\mathrm{obs}}$. Furthermore, the CIBA at $3000 \mathrm{GHz}$ and the CIBA in the Planck bands are weakly correlated, so that $T_{\text {obs }}$ contains most of the information on the CIBA at $3000 \mathrm{GHz}$. Therefore, through $I_{353}$ and $B_{353}\left(T_{\text {obs }}\right)$, the map of $\tau_{353}$ is affected by the CIBA on both the Rayleigh-Jeans and Wien sides, respectively, resulting in strong small scale fluctuations. On the other hand, because $\mathcal{R}$ is obtained by integrating $I_{v}$ over frequency, it benefits from the fact that the CIBA decorrelates in frequency; i.e., the integral over frequency of the CIBA is close to zero.

In order to relate Planck dust emission to Galactic reddening (Sect. 6), we also made a fit of the dust model to a version of the Planck and IRAS data from which point sources had been

7 For example, a larger number of bands could reveal that a singletemperature MBB is not an adequate fitting function, a conclusion that cannot be reached with the four bands used here. 


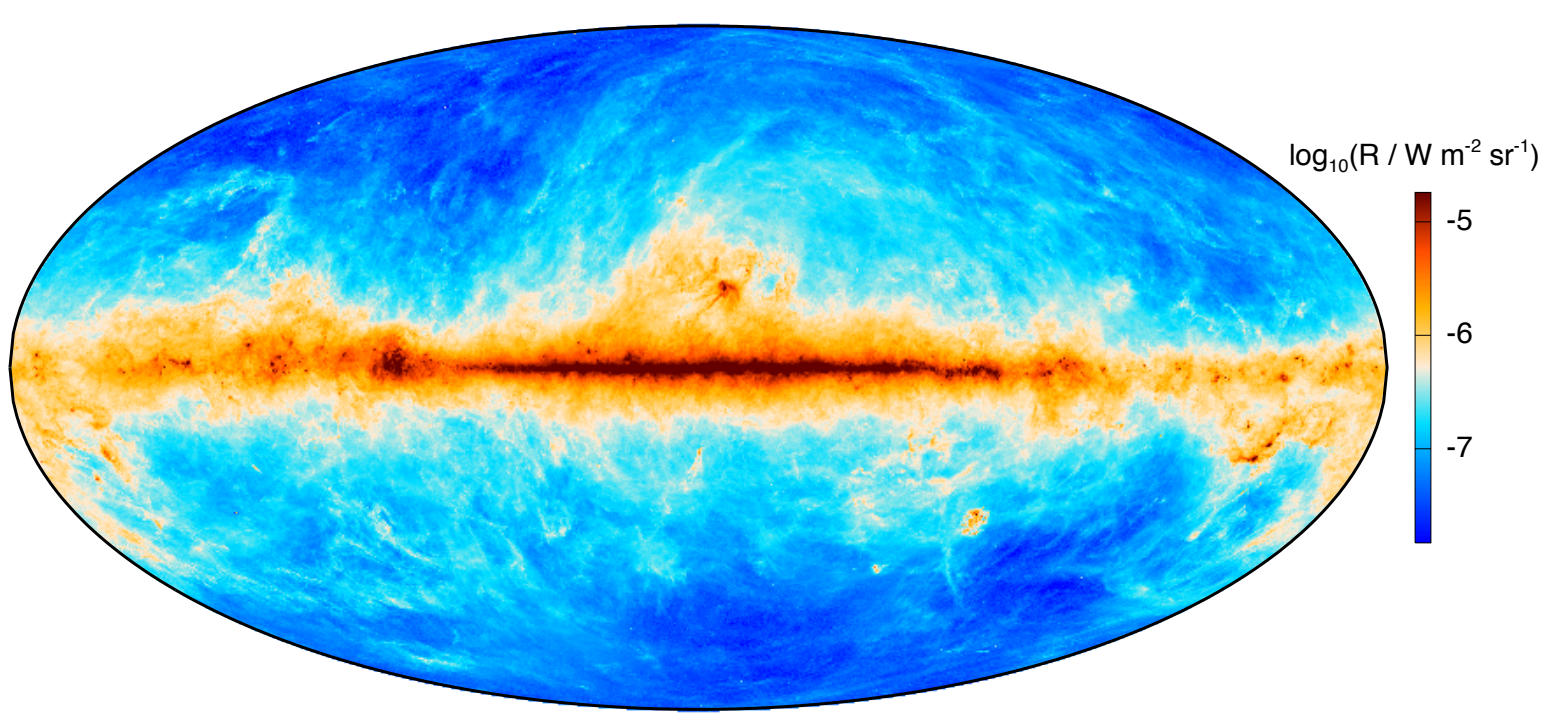

Fig. 12. All-sky map of dust radiance at $5^{\prime}$ resolution. The range shown corresponds to $-7.8<\log _{10}(\mathcal{R})<-4.7$.
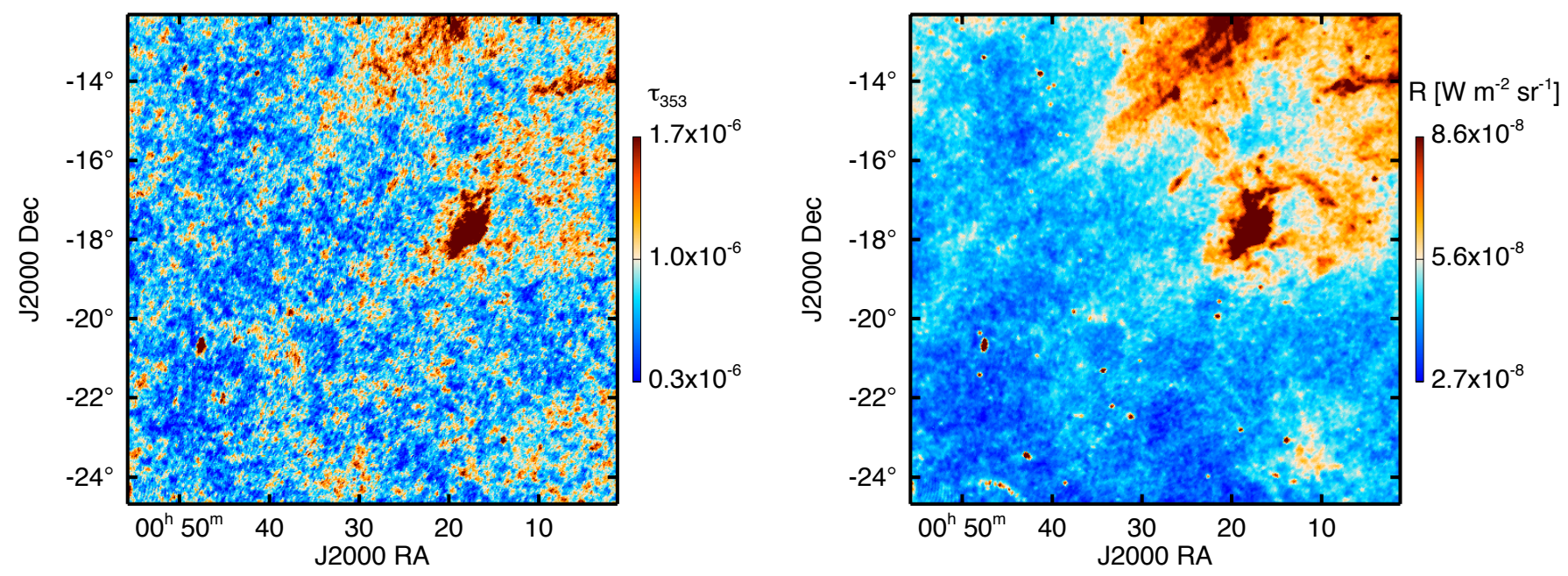

Fig. 13. Maps of $\tau_{353}$ (left) and $\mathcal{R}$ (right) of a diffuse area of the sky, centred on $l=90^{\circ}, b=-80^{\circ}$.

removed (Appendix D). From this fit we have also made maps of $\tau_{353}$ and $\mathcal{R}$.

\section{The Galactic dust emission observed by Planck}

\subsection{Spatial variations of the dust parameters and $\mathcal{R}$}

The all-sky maps of $T_{\text {obs }}, \beta_{\text {obs }}$, and $\tau_{353}$ in Fig. 9 - represented as well in a polar orthographic projection in in Figs. 14 and 15 to show details in the high-latitude sky - represent the first attempt to fit these three parameters at the same time over the whole sky. Together, these maps of the fit parameters provide information on the dust SED and, quite likely, on the dust properties and their variations with interstellar environment. They are complemented by the map of $\mathcal{R}$ in Figs. 12 and 14. Here we discuss only some broad features of these maps, leaving more detailed analysis to future work.

The mean and standard deviation of $T_{\text {obs }}, \beta_{\text {obs }}, \tau_{353}$, and $\mathcal{R}$ are given in Table 3 for several different masks ranked in order of decreasing dust contamination, using $\sigma\left(\tau_{353}\right)$ as a proxy, and so (mostly) of decreasing sky coverage. These include some masks used in Planck cosmology papers (e.g., Planck Collaboration XV 2014). Note how the ranking is reflected in the means and standard deviations listed.

Over the whole sky, the mean of $\beta_{\mathrm{obs}}$ is 1.62 and its standard deviation is 0.10 . The mean of $T_{\mathrm{obs}}$ is $19.7 \mathrm{~K}$ and its standard deviation is $1.4 \mathrm{~K}$. The distribution function of $T_{\mathrm{obs}}$ is slightly positively skewed with a high tail that extends up to $60 \mathrm{~K}$. Only about 100 out of the more than 50 million pixels of the $N_{\text {side }}=$ 2048 map have $T_{\text {obs }}<13 \mathrm{~K}^{8}$.

The maps of $T_{\mathrm{obs}}$ and $\tau_{353}$ presented here should be compared with the ones published as Planck early results by Planck Collaboration XIX (2011). Apart from the facts that we use a more recent release of Planck data (with a different calibration of the 545 and $857 \mathrm{GHz}$ and with $\mathrm{ZE}$ removed) and a slightly

\footnotetext{
8 This is not in contradiction with the cold clumps detected in the Planck data (Planck Collaboration XXIII 2011), some with temperature as low as $7 \mathrm{~K}$. These clumps were identified after removing a hotter background/foreground emission. We do not obtain such low values of $T_{\text {obs }}$ because we model the observed specific intensity on each line of sight.
} 

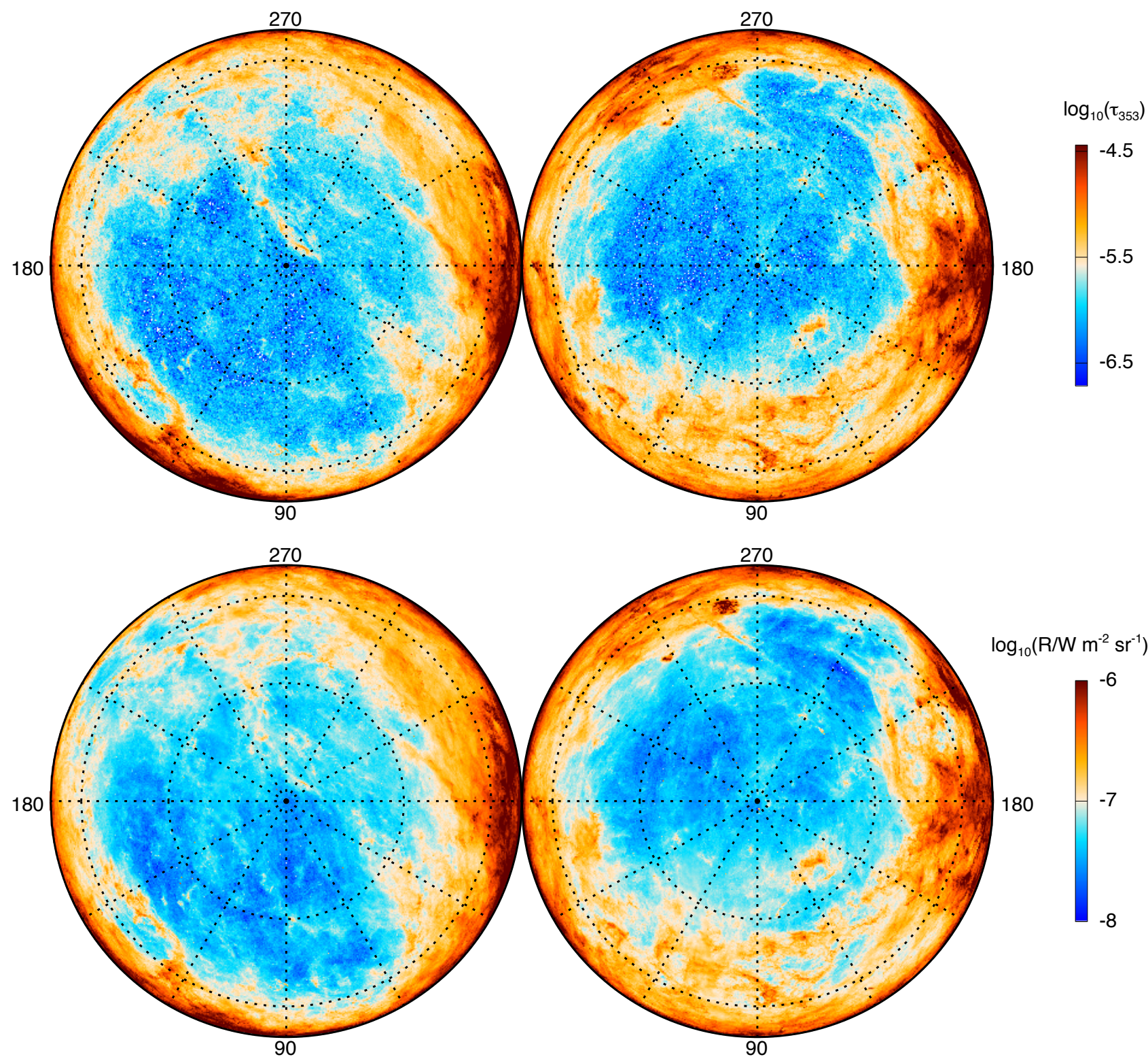

Fig. 14. Polar views of $\log _{10}\left(\tau_{353}\right)$ (upper) and $\log _{10}(\mathcal{R})$ (lower).

different approach to the offset determination ${ }^{9}$, the main difference is that we fit for $\beta_{\text {obs }}$ while Planck Collaboration XIX (2011) used a fixed value, $\beta_{\text {obs }}=1.8$, a convention shared among all the Planck Early Papers dedicated to dust emission (Planck Collaboration XXV 2011; Planck Collaboration XXIV 2011; Planck Collaboration XXI 2011). Even with these differences in data and methodology, the maps of $T_{\text {obs }}$ are remarkably similar. The map of $T_{\text {obs }}$ presented here is higher by about $1 \mathrm{~K}$ than that of Planck Collaboration XIX (2011), due principally to the modification of the calibration of the 545 and $857 \mathrm{GHz}$ channels.

Like in Planck Collaboration XIX (2011), the lowest $T_{\text {obs }}$ values are found in the outer Galaxy and in molecular clouds. In general the well-known molecular clouds have a lower $T_{\text {obs }}(15-17 \mathrm{~K})$ and higher $\beta_{\text {obs }}$ (around 1.8) than in the diffuse ISM. This trend is compatible with the result of Planck Collaboration XXV (2011) who reported a steepening of

\footnotetext{
9 Both studies use the correlation with H I to set the offsets but Planck Collaboration XIX (2011) used a higher threshold in column density $\left(N_{\mathrm{HI}}<1.2 \times 10^{21} \mathrm{~cm}^{-2}\right)$ than adopted here $\left(N_{\mathrm{HI}}<2 \times 10^{20} \mathrm{~cm}^{-2}\right)$.
}

the SED from diffuse to molecular areas in the Taurus molecular cloud.

Small-scale regions of higher $T_{\text {obs }}$ are seen along the Galactic plane and in many of the Gould Belt clouds, most probably related to the local production of dust-heating photons in Galactic star forming regions. The Magellanic Clouds are clearly visible in the parameter maps with a higher $T_{\text {obs }}$ and lower $\beta_{\text {obs }}$ (Planck Collaboration XVII 2011).

The main noticeable difference with respect to the early results of Planck Collaboration XIX (2011) is the lower $T_{\text {obs }}$ found here in the inner Galactic plane. This is due to the fact that we fit for $\beta_{\text {obs }}$, which appears to have a systematically higher value in the inner Galactic plane, in the range 1.8-2.0. The impact of noise and the CIBA is obviously negligible in this bright area of the sky. The higher $\beta_{\text {obs }}$ found here clearly provides a better representation of the SED, as shown also by Planck Collaboration Int. XIV (2014). This steepening of the dust SED in the inner Galactic plane is also compatible with the analysis of Herschel observations of that region by Paradis et al. (2012). 

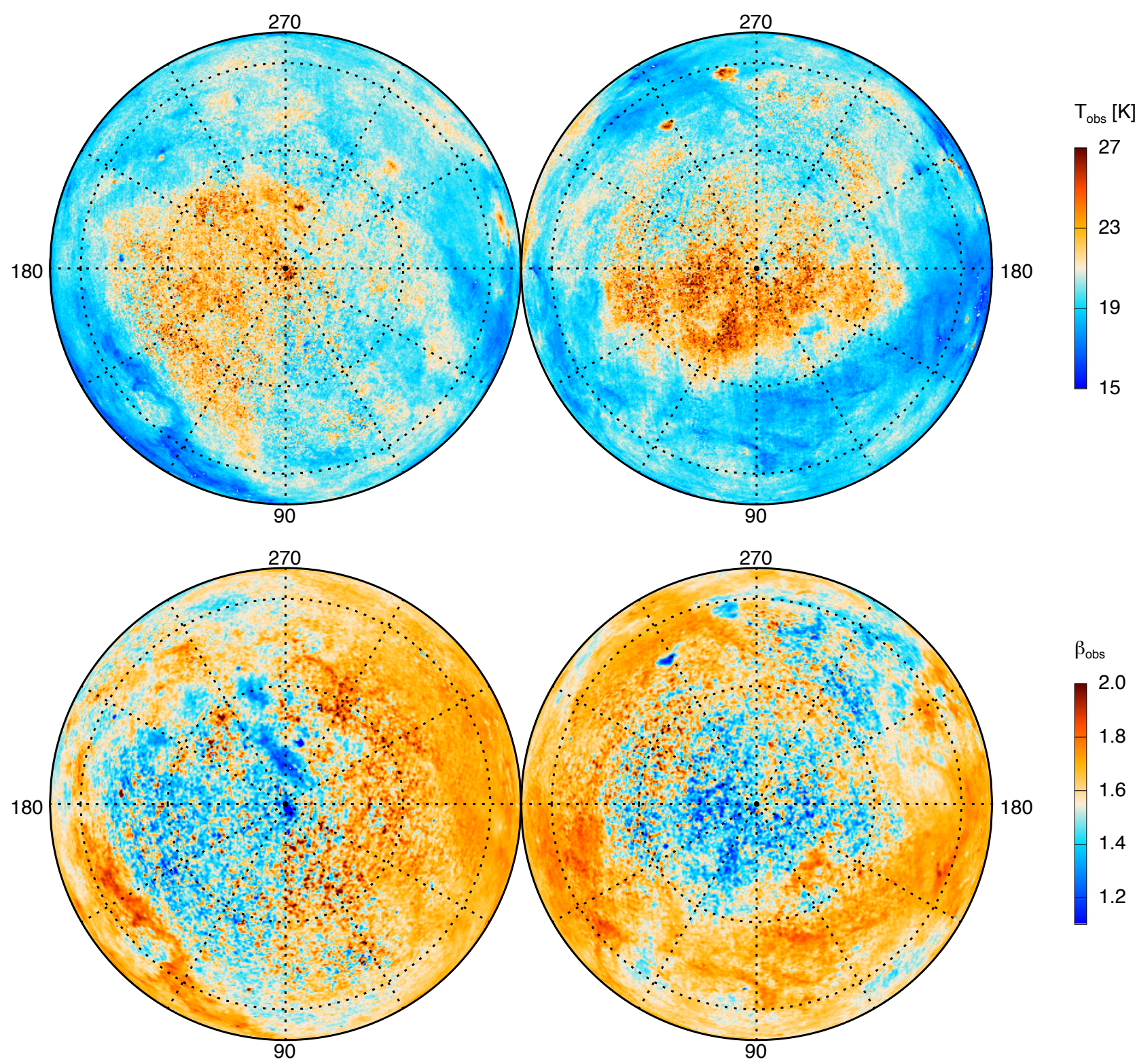

Fig. 15. Polar views of $T_{\mathrm{obs}}$ (upper) and $\beta_{\mathrm{obs}}$ (lower).

Table 3. Summary of the mean and standard deviation of the dust parameters for specific masks.

\begin{tabular}{lccccccccc}
\hline \hline Mask & $\begin{array}{c}\text { Coverage } \\
{[\%]}\end{array}$ & $\begin{array}{c}\left\langle T_{\text {obs }}\right\rangle \\
{[\mathrm{K}]}\end{array}$ & $\begin{array}{c}\sigma\left(T_{\text {obs }}\right) \\
{[\mathrm{K}]}\end{array}$ & $\left\langle\beta_{\text {obs }}\right\rangle$ & $\sigma\left(\beta_{\text {obs }}\right)$ & $\left\langle\tau_{353}\right\rangle$ & $\sigma\left(\tau_{353}\right)$ & $\begin{array}{c}\langle\mathcal{R}\rangle \\
{\left[\mathrm{W} \mathrm{m}^{-2} \mathrm{sr}^{-1}\right]}\end{array}$ & $\begin{array}{c}\sigma(\mathcal{R}) \\
{\left[\mathrm{W} \mathrm{m}^{-2} \mathrm{sr}^{-1}\right]}\end{array}$ \\
\hline Whole sky & 100 & 19.7 & 1.4 & 1.62 & 0.10 & $45.0 \times 10^{-7}$ & $53.3 \times 10^{-7}$ & $15.2 \times 10^{-8}$ & $16.9 \times 10^{-8}$ \\
G56 & 57 & 20.2 & 1.2 & 1.60 & 0.12 & $21.7 \times 10^{-7}$ & $16.9 \times 10^{-7}$ & $8.0 \times 10^{-8}$ & $5.3 \times 10^{-8}$ \\
$|b|>15^{\circ}$ & 50 & 20.3 & 1.3 & 1.59 & 0.12 & $18.5 \times 10^{-7}$ & $13.2 \times 10^{-7}$ & $7.1 \times 10^{-8}$ & $4.1 \times 10^{-8}$ \\
$\mathrm{G} 45$ & 47 & 20.3 & 1.3 & 1.59 & 0.12 & $17.5 \times 10^{-7}$ & $12.7 \times 10^{-7}$ & $6.9 \times 10^{-8}$ & $4.0 \times 10^{-8}$ \\
$\mathrm{G} 35$ & 37 & 20.5 & 1.3 & 1.57 & 0.13 & $13.8 \times 10^{-7}$ & $8.8 \times 10^{-7}$ & $5.7 \times 10^{-8}$ & $2.8 \times 10^{-8}$ \\
South cap & 17 & 20.5 & 1.4 & 1.59 & 0.13 & $14.5 \times 10^{-7}$ & $10.7 \times 10^{-7}$ & $6.2 \times 10^{-8}$ & $3.6 \times 10^{-8}$ \\
Low $N_{\mathrm{H} \text { I }}$ & 11 & 20.8 & 1.4 & 1.55 & 0.15 & $9.6 \times 10^{-7}$ & $4.1 \times 10^{-7}$ & $4.1 \times 10^{-8}$ & $1.2 \times 10^{-8}$ \\
Lowest 1\% & 1 & 20.9 & 1.7 & 1.51 & 0.18 & $6.4 \times 10^{-7}$ & $2.9 \times 10^{-7}$ & $2.5 \times 10^{-8}$ & $0.5 \times 10^{-8}$ \\
\hline
\end{tabular}

Notes. The angular resolution of all quantities is $5^{\prime}$ except for $\beta_{\mathrm{obs}}$ which is at $30^{\prime}$. The $|b|>15^{\circ}$ mask also includes the restriction $N_{\mathrm{HI}}<$ $5.5 \times 10^{20} \mathrm{~cm}^{-2}$. The Low $N_{\mathrm{HI}}$ mask is the one shown in Fig. 3, left. The south cap mask corresponds to that developed for the analysis in Planck Collaboration Int. XVII (2014). The Lowest $1 \%$ mask corresponds to the lowest $1 \% N_{\mathrm{HI}}$ column density estimated using the LAB data. The remaining masks (G35, G45, and G56) are among those used in the Planck cosmology papers (e.g., Planck Collaboration XV 2014), based in part on thresholding the Planck $353 \mathrm{GHz}$ temperature map. 
One striking feature of the $T_{\text {obs }}$ (polar) map is the increase toward both Galactic poles. Selecting the pixels corresponding to the lowest $1 \% N_{\mathrm{HI}}$, the mean $T_{\mathrm{obs}}$ is $20.9 \mathrm{~K}$ and the mean $\beta_{\mathrm{obs}}$ is 1.51. This systematic increase of $T_{\text {obs }}$ was also visible in the early all-sky map of Planck Collaboration XIX (2011) that used a constant $\beta_{\text {obs }}$, different offsets, a different $3000 \mathrm{GHz}$ map and no $\mathrm{ZE}$ removal for the Planck data. In addition, the values we report for the south Galactic pole mask (mean $T_{\mathrm{obs}}=20.5 \mathrm{~K}$, $\sigma\left(T_{\text {obs }}\right)=1.4 \mathrm{~K}$, mean $\left.\beta_{\text {obs }}=1.59, \sigma\left(\beta_{\text {obs }}\right)=0.13\right)$ are compatible with the ones reported by Planck Collaboration Int. XVII (2014) using a correlation method that is insensitive to offsets and ZE removal. The nature of this increase of $T_{\text {obs }}$ over a large scale in the most diffuse areas at high Galactic latitudes, incidentally correlated with lower values of $\beta_{\text {obs }}$ (see Fig. 15), is still to be understood (see Sect. 5.2) but it is unlikely to be caused by a bias by instrumental noise or the CIBA, which both create small-scale fluctuations.

\section{2. $\beta_{\text {obs }}-T_{\text {obs }}$ relation}

The all-sky maps of the fit parameters (Fig. 9) reveal some spatial correlation between the parameters. This is especially clear between $\beta_{\text {obs }}$ and $T_{\text {obs }}$ as illustrated in Fig. 16, lower, using results for all pixels on the sky. Because it includes so many different regions, this two-dimensional histogram can reveal only global trends, here the general anticorrelation.

This anticorrelation is visible in the faintest parts of the sky, at both small and large scales. It is also seen at the scale of clouds; the Gould Belt clouds have a low $T_{\text {obs }}(15-16 \mathrm{~K})$ and high $\beta_{\text {obs }}(\sim 1.8)$. Several other studies have highlighted similar $\beta_{\text {obs }}-T_{\text {obs }}$ anticorrelations from observations of specific regions on the sky (Dupac et al. 2003; Désert et al. 2008; Paradis et al. 2010; Planck Collaboration XXV 2011). On the other hand, this behaviour does not extend to the Galactic plane where the two parameters seem to be more correlated than anticorrelated.

As pointed out in Sect. 3.2 (see also Shetty et al. 2009b), instrumental noise is an obvious candidate that might create a $\beta-T$ anticorrelation. However, the fractional variations of $\beta_{\mathrm{obs}}$ and $T_{\text {obs }}$ observed here over most of the sky significantly exceed the statistical uncertainties of these parameters taking into account noise and calibration uncertainties (see Fig. 10).

On the other hand, as shown in Sect. 3.2.2 and Appendix C, for faint dust emission the CIBA can produce significant variations of $T_{\text {obs }}$ and $\beta_{\text {obs }}$ at small scales, and although this effect is in fact observed, it is not accounted for in the error budget. To be quantitative, in the pixels corresponding to the lowest $1 \%$ values of $N_{\mathrm{HI}}$ the observed standard deviations of these parameters are the largest $-\sigma\left(T_{\text {obs }}\right)=1.7 \mathrm{~K}$ and $\sigma\left(\beta_{\text {obs }}\right)=0.18-$ while over the whole sky $\sigma\left(T_{\text {obs }}\right)=1.4 \mathrm{~K}$ and $\sigma\left(\beta_{\text {obs }}\right)=0.10$ (see Table 3$)$. Based on the Monte-Carlo simulations presented in Sect. 3.2.3, for values of $\tau_{353}$ typical of the faintest $1 \%$ pixels of the sky the noise and CIBA produce fluctuations of $T_{\mathrm{obs}}$ and $\beta_{\mathrm{obs}}$ of the order of $\delta T_{\text {obs }}=1.7 \mathrm{~K}$ and $\delta \beta_{\text {obs }}=0.15$, providing a credible explanation for the magnitude of the small-scale variations observed in that mask (Table 3).

Even though noise and the CIBA seem to be responsible for the $\beta-T$ anticorrelation in the most diffuse areas of the sky, they can cause only small-scale fluctuations because of their flat power spectra. Because the monopole of the CIB was removed from the map, the CIBA does not bias $\beta_{\text {obs }}$ and $T_{\text {obs }}$ globally on the sky, and cannot produce large-scale variations like the increase of $T_{\mathrm{obs}}$ toward the Galactic poles. We have also checked that these results are largely unaffected by the ZE removal (Appendix A.2).
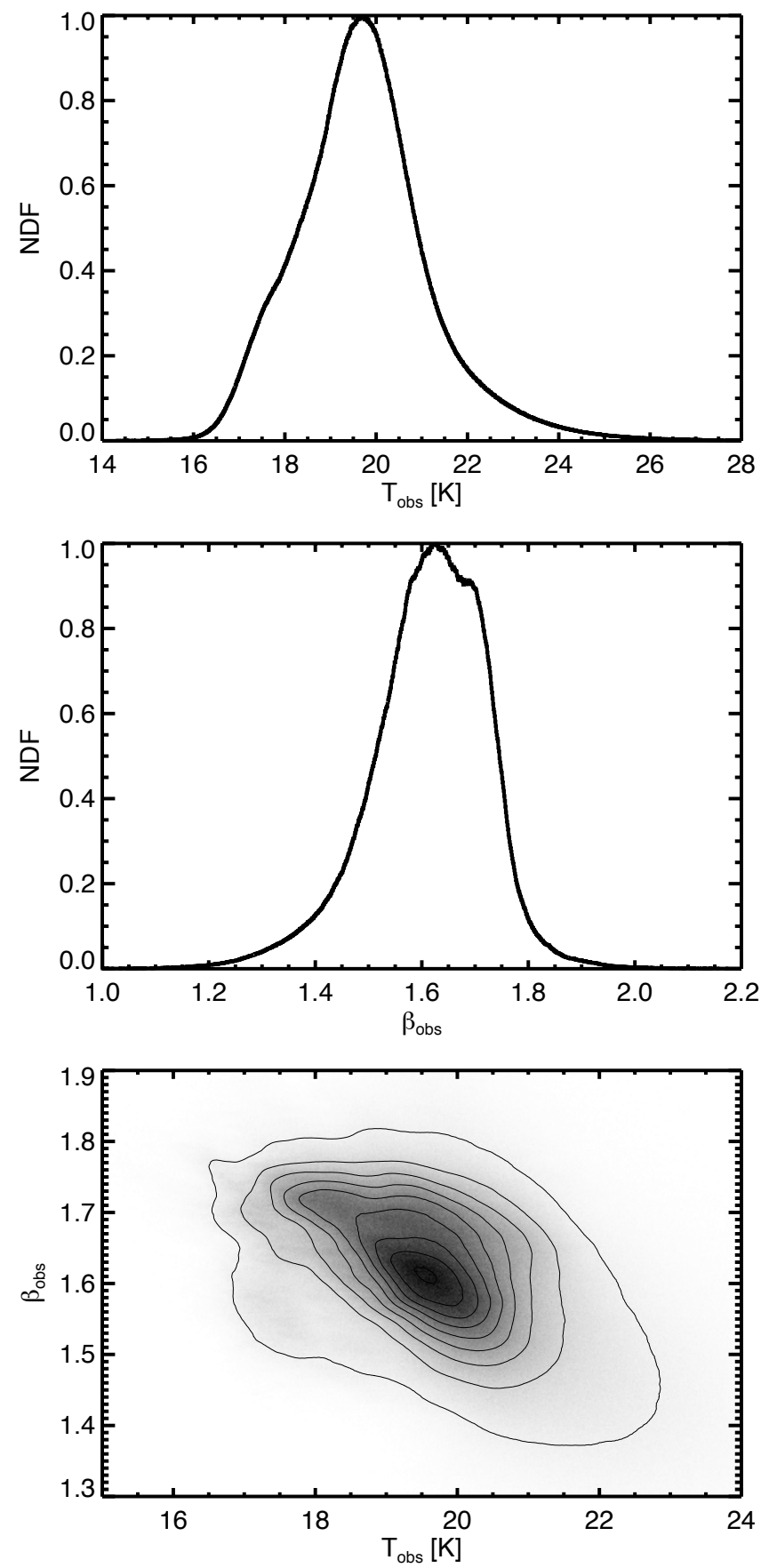

Fig. 16. Normalized distribution function of $T_{\mathrm{obs}}$ (upper) and $\beta_{\mathrm{obs}}$ (middle) for the whole sky. The lower panel shows the density plot of $\beta_{\mathrm{obs}}$ vs. $T_{\mathrm{obs}}$, revealing an overall anticorrelation. The grey scale and the black contours show the density of points on a linear scale with contours equally spaced. The dispersions are $\sigma\left(\beta_{\text {obs }}\right)=0.1$ and $\sigma\left(T_{\text {obs }}\right)=1.4 \mathrm{~K}$ (Table 3).

In brighter regions, our Monte-Carlo simulations (Sect. 3.2.3) indicate that noise and the CIBA introduce variations in $T_{\mathrm{obs}}$ and $\beta_{\mathrm{obs}}$ (Fig. 8) that are below the observed dispersions (Fig. 16, lower). This is true for more than $90 \%$ of the sky. One can appreciate these results by looking directly at the parameter maps (Fig. 9). Away from the most diffuse areas of the sky, where $T_{\mathrm{obs}}$ and $\beta_{\mathrm{obs}}$ vary at small scale mostly due to the CIBA, the main clouds and interstellar structures that are 
Table 4. Summary of the mean and standard deviation of the dust opacity and dust specific luminosity for different masks.

\begin{tabular}{lcccc}
\hline \hline Mask & $\begin{array}{c}\left\langle\sigma_{\mathrm{e} 353}\right\rangle \\
{\left[\mathrm{cm}^{2} \mathrm{H}^{-1}\right]}\end{array}$ & $\begin{array}{c}\sigma\left(\sigma_{\mathrm{e} 353}\right) \\
{\left[\mathrm{cm}^{2} \mathrm{H}^{-1}\right]}\end{array}$ & $\begin{array}{c}\left\langle L_{\mathrm{H}}\right\rangle \\
{\left[\mathrm{W} \mathrm{H}^{-1}\right]}\end{array}$ & $\begin{array}{c}\sigma\left(L_{\mathrm{H}}\right) \\
{\left[\mathrm{W} \mathrm{H}^{-1}\right]}\end{array}$ \\
\hline Whole sky & $8.4 \times 10^{-27}$ & $3.0 \times 10^{-27}$ & $3.5 \times 10^{-31}$ & $0.9 \times 10^{-31}$ \\
G56 & $7.1 \times 10^{-27}$ & $1.9 \times 10^{-27}$ & $3.4 \times 10^{-31}$ & $0.6 \times 10^{-31}$ \\
$|b|>15^{\circ}$ & $7.0 \times 10^{-27}$ & $2.0 \times 10^{-27}$ & $3.4 \times 10^{-31}$ & $0.6 \times 10^{-31}$ \\
G45 & $6.8 \times 10^{-27}$ & $1.8 \times 10^{-27}$ & $3.3 \times 10^{-31}$ & $0.6 \times 10^{-31}$ \\
G35 & $6.5 \times 10^{-27}$ & $1.8 \times 10^{-27}$ & $3.3 \times 10^{-31}$ & $0.6 \times 10^{-31}$ \\
South cap & $6.5 \times 10^{-27}$ & $1.9 \times 10^{-27}$ & $3.4 \times 10^{-31}$ & $0.5 \times 10^{-31}$ \\
Low $N_{\mathrm{H}}$ & $6.6 \times 10^{-27}$ & $1.7 \times 10^{-27}$ & $3.5 \times 10^{-31}$ & $0.6 \times 10^{-31}$ \\
Lowest 1\% & $7.9 \times 10^{-27}$ & $1.9 \times 10^{-27}$ & $3.8 \times 10^{-31}$ & $0.7 \times 10^{-31}$ \\
\hline
\end{tabular}

Notes. All quantities were computed using maps at $30^{\prime}$ resolution. The map of $N_{\mathrm{H}}$ is a combination of H I (21 cm LAB data) and CO (Planck) assuming $X_{\mathrm{CO}}=2 \times 10^{20} \mathrm{H}_{2} \mathrm{~cm}^{-2} \mathrm{~K}^{-1} \mathrm{~km}^{-1} \mathrm{~s}$ following Bolatto et al. (2013). This estimate of $N_{\mathrm{H}}$ is a lower limit as it does not account for the ionized gas and the molecular gas not detected via CO. See Table 3 for the definitions of each mask.

seen in $I_{v}$ and in $\tau_{353}$ can be recognized in the maps of $T_{\mathrm{obs}}$ and $\beta_{\text {obs. }}$.

The broad spectral coverage, the high signal-to-noise (and high signal-to-CIBA) ratio of the data on more than $90 \%$ of the sky, and the methodology used to minimize the effects of noise and the CIBA on the fit parameters, combine to produce values of $T_{\text {obs }}$ and $\beta_{\text {obs }}$ with uncertainties of a few percent, much smaller than their dispersions over the sky (Table 3 ). We conclude that on most of the sky, the relation between $\beta_{\mathrm{obs}}$ and $T_{\mathrm{obs}}$ is not an artifact of the data processing (zero levels, ZE correction) or due to noise or the CIBA. This conclusion also holds for the largescale variations of $\beta_{\mathrm{obs}}$ and $T_{\mathrm{obs}}$ at high Galactic latitude. On most of the sky, the systematic variations of $T_{\mathrm{obs}}$ and $\beta_{\mathrm{obs}}$ are related to real changes in the shape of SED of the interstellar dust emission.

Even with data-related effects mitigated, the interpretation of the relationship between the MBB parameters is complex. In particular at this point it is difficult to be definitive about the origin of the relationship between $T_{\mathrm{obs}}$ and $\beta_{\mathrm{obs}}$. It depends on details of radiative transfer, of variations in $U$ along the line of sight, and of variations in grain structure and size distribution. To identify the relative roles of dust evolution and line-of-sight integration effects in this observed phenomenon, detailed studies of specific spatially-coherent objects in various interstellar environments and at all scales are needed.

\subsection{Dust SED in the diffuse ISM}

As described in Planck Collaboration VIII (2014), the calibration scheme for the 545 and $857 \mathrm{GHz}$ data has changed since the Planck Early Results. These channels are no longer calibrated using the FIRAS data, but instead rely on observations of planets as for IRAS, DIRBE, and Herschel. Compared to the previous situation, the calibration factor has been divided by 1.15 at $545 \mathrm{GHz}$ and 1.07 at $857 \mathrm{GHz}$ (Planck Collaboration VIII 2014), so that the specific intensities are now lower.

There are two main impacts on dust modelling. First, the shape of the dust SED is modified, changing the average $T_{\text {obs }}$ and $\beta_{\text {obs }}$. The FIRAS average dust SED of the diffuse ISM mask $\left(|b|>15^{\circ}\right.$ and $N_{\mathrm{HI}}<5.5 \times 10^{20} \mathrm{~cm}^{-2}$, following the definition of Compiègne et al. 2011) was modelled with $T_{\mathrm{obs}}=17.9 \mathrm{~K}$ and $\beta_{\text {obs }}=1.84$ by Planck Collaboration XXIV (2011), compatible with the average SED that they found in selected high Galactic latitude fields using IRAS and the early Planck data ${ }^{10}$. With

\footnotetext{
10 This was expected because the 857,545 , and $353 \mathrm{GHz}$ data used in that study were calibrated on FIRAS.
}

the new calibration, the mean values found for the same mask are significantly different: $\left\langle T_{\text {obs }}\right\rangle=20.3 \mathrm{~K}$ and $\left\langle\beta_{\text {obs }}\right\rangle=1.59$ (see Table 3). The dust parameters found here are similar to those found in external galaxies with Herschel ${ }^{11}$, even though the Herschel frequency coverage is not as extensive (e.g., Dale et al. 2012).

The second impact is on the value of the dust opacity $\sigma_{\mathrm{e} v}=\tau_{v} / N_{\mathrm{H}}$. The increase in $T_{\mathrm{obs}}$ due to the recalibration lowers $\tau_{v}$ and the opacity. At $250 \mu \mathrm{m}(1200 \mathrm{GHz})$, a reference wavelength often used, Boulanger et al. (1996) obtained $\sigma_{\mathrm{e} 1200}=1.0 \times 10^{-25} \mathrm{~cm}^{2}$ while here for the $|b|>15^{\circ}$ mask we obtain $\sigma_{\mathrm{e} 1200}=0.49 \times 10^{-25} \mathrm{~cm}^{2}$ (from $\sigma_{\mathrm{e} 353}$ in Table 4 and $\beta_{\text {obs }}=1.59$ ).

Changing $\beta_{\text {obs }}$ directly affects the assessment of the material needed to explain the observed thermal emission; in a MBB fit to the SED, a lower $\beta_{\text {obs }}$ leads to a higher $T_{\text {obs }}$ and therefore to a lower optical depth, which in turn could be interpreted as a lower column density (or mass), or a lower opacity. We also note that the mean value of $\beta_{\text {obs }}$ found is lower than used for some components in dust models, like graphite in Draine \& Li (2007) where $\beta=2$; when fitting with such a model, a higher radiation field strength $U$ would be needed.

\section{Dust emission in relation to gas column density}

In the previous section we have described the properties of the parameters that define the dust SED. Now we concentrate on the link between the dust emission and the interstellar gas column density, following on many detailed studies in environments from the diffuse ISM (Boulanger \& Pérault 1988; Boulanger et al. 1996) to molecular clouds (Pineda et al. 2008; Goodman et al. 2009).

Here the estimate of gas column density, $N_{\mathrm{H}}$, accounts for atomic and molecular gas:

$N_{\mathrm{H}}=N_{\mathrm{HI}}+2 X_{\mathrm{CO}} W_{\mathrm{CO}}$,

where the $N_{\mathrm{H} \text { I }}$ is from the LAB data assuming optically-thin emission, $W_{\mathrm{CO}}$ is from the Planck ${ }^{12} \mathrm{CO} J=1 \rightarrow 0$ map (type 3 ) (Planck Collaboration XIII 2014), and $X_{\mathrm{CO}}$ is not constant but is typically $2 \times 10^{20} \mathrm{H}_{2} \mathrm{~cm}^{-2} \mathrm{~K}^{-1} \mathrm{~km}^{-1} \mathrm{~s}$ (Bolatto et al. 2013). "Dark" neutral matter (Planck Collaboration XIX 2011) is by

11 The calibration of each of Herschel and Planck at 545 and $857 \mathrm{GHz}$ is based on observations of planets and uses the same model of planetary emission (Planck Collaboration VIII 2014). 

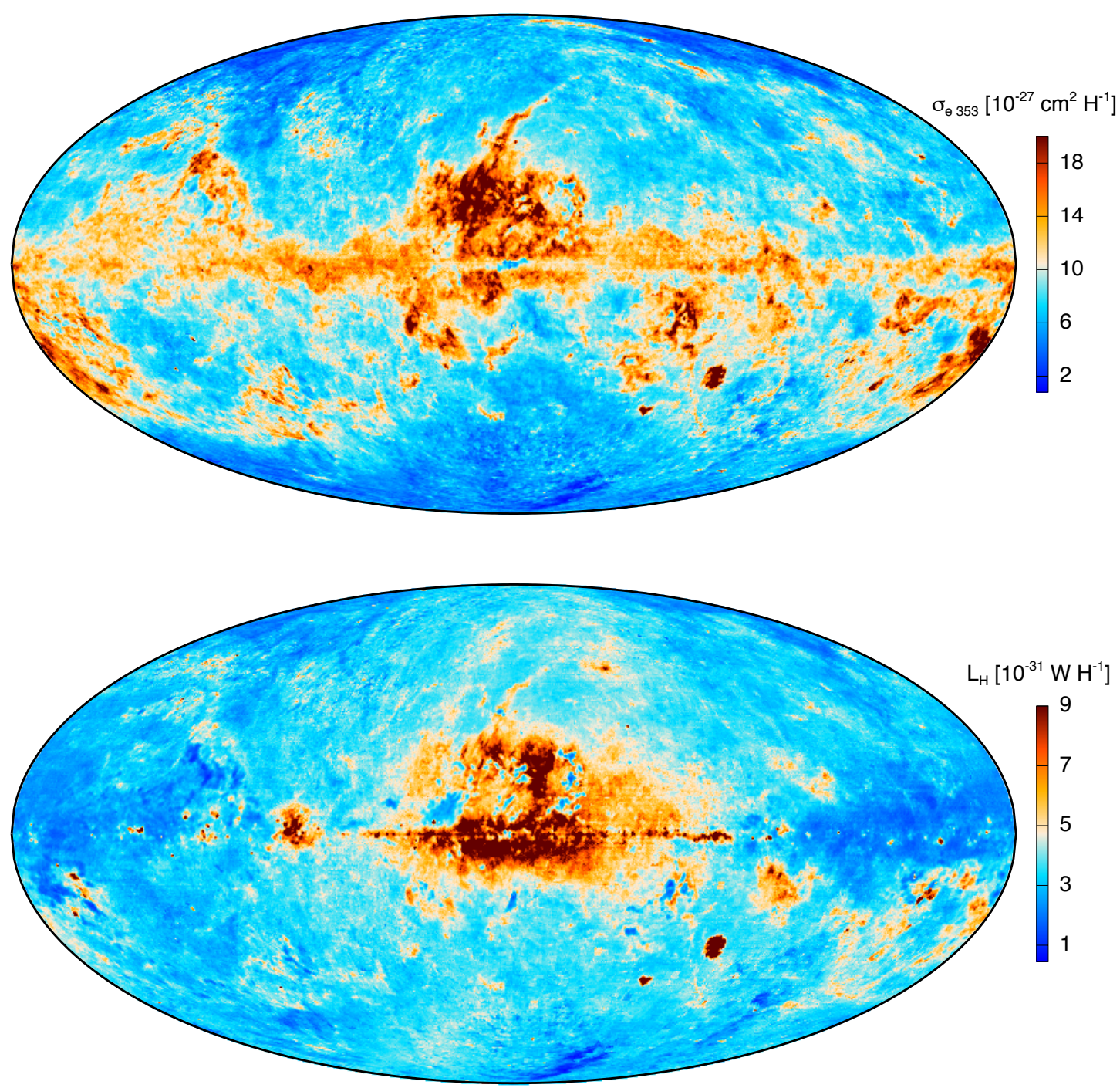

Fig. 17. All-sky maps of $\sigma_{\mathrm{e} 353}$ (upper) and $L_{\mathrm{H}}$ (lower). The gas column density $N_{\mathrm{H}}$ is $N_{\mathrm{HI}}+2 X_{\mathrm{CO}} W_{\mathrm{CO}}$ where $N_{\mathrm{HI}}$ is from the LAB data, $W_{\mathrm{CO}}$ is from the Planck ${ }^{12} \mathrm{CO} J=1 \rightarrow 0$ map (type 3 ), and $X_{\mathrm{CO}}=2 \times 10^{20} \mathrm{H}_{2} \mathrm{~cm}^{-2} \mathrm{~K}^{-1} \mathrm{~km}^{-1} \mathrm{~s}$.

definition left out in this formulation, though it is among the total that can be traced by $\gamma$-rays (Grenier et al. 2005). Ionized gas is left out for lack of a proper template.

\subsection{Opacity and dust specific luminosity}

The optical depth $\left(\tau_{353}\right.$ here) is often taken as a tracer of $N_{\mathrm{H}}$ but this is only accurate if the opacity is constant (Eq. (3)). This requirement can be assessed in the all-sky map of the opacity $\sigma_{\mathrm{e} 353}=\tau_{353} / N_{\mathrm{H}}$ in Figs. 17 and 18, smoothed to 30'. Although the large dynamic range over the $\tau_{353}$ sky is greatly compressed, so that a linear scale can be used, it is clear that there are changes in opacity, even in the diffuse atomic ISM in the high-latitude sky where $N_{\mathrm{H}}$ is well measured. Related to these changes in opacity are changes in the equilibrium dust temperature (Planck Collaboration XXIV 2011; Planck Collaboration Int. XVII 2014), driving complementary changes in the SED parameter $\tau_{353}$ through Eq. (6). This demonstrates how $\tau_{353}$ is compromised as a tracer of column density.

We saw in Sect. 3.4 how $\mathcal{R}$ compensates for such effects, being smoother than $\tau_{353}$. This is expected to carry over into the dust specific luminosity

$L_{\mathrm{H}}=4 \pi \mathcal{R} / N_{\mathrm{H}}$,

also shown as an all-sky map in Figs. 17 and 18. At high latitudes this is indeed more uniform. This uniformity and the excursions to both higher and lower values at higher column densities relating to the ambient ISRF are taken up in Sect. 5.2.

Complementing the above, for low-column-density lines of sight with $1 \times 10^{20}<N_{\mathrm{H}}<2.5 \times 10^{20} \mathrm{~cm}^{-2}$, the dependence of $\sigma_{\mathrm{e} 353}$ on $T_{\mathrm{obs}}$, and by contrast the relative lack of dependence of $L_{\mathrm{H}}$ on $T_{\mathrm{obs}}$, are evident in Fig. 19, lower.

The statistics of $\sigma_{\mathrm{e} 353}$ and $L_{\mathrm{H}}$ for the various masks are presented in Table 4 using $X_{\mathrm{CO}}=2 \times 10^{20} \mathrm{H}_{2} \mathrm{~cm}^{-2} \mathrm{~K}^{-1} \mathrm{~km}^{-1} \mathrm{~s}$ where relevant ${ }^{12}$. Note how for these normalized quantities the systematic ranking seen in Table 3 is not preserved.

12 Use of a constant $X_{\mathrm{CO}}$ is certainly not realistic, given the large ranges in density and temperature covered. On the other hand, it is used here only to provide basic statistics of $\sigma_{\mathrm{e} 353}$ and $L_{\mathrm{H}}$ for the "Whole sky" mask in Table 4 . The sky fraction with significant $\mathrm{CO}$ emission, greater than $0.15 \mathrm{~K} \mathrm{~km} \mathrm{~s}^{-1}$, is only about $18 \%$ and in all of the other masks considered here $\mathrm{CO}$ does not contribute. 

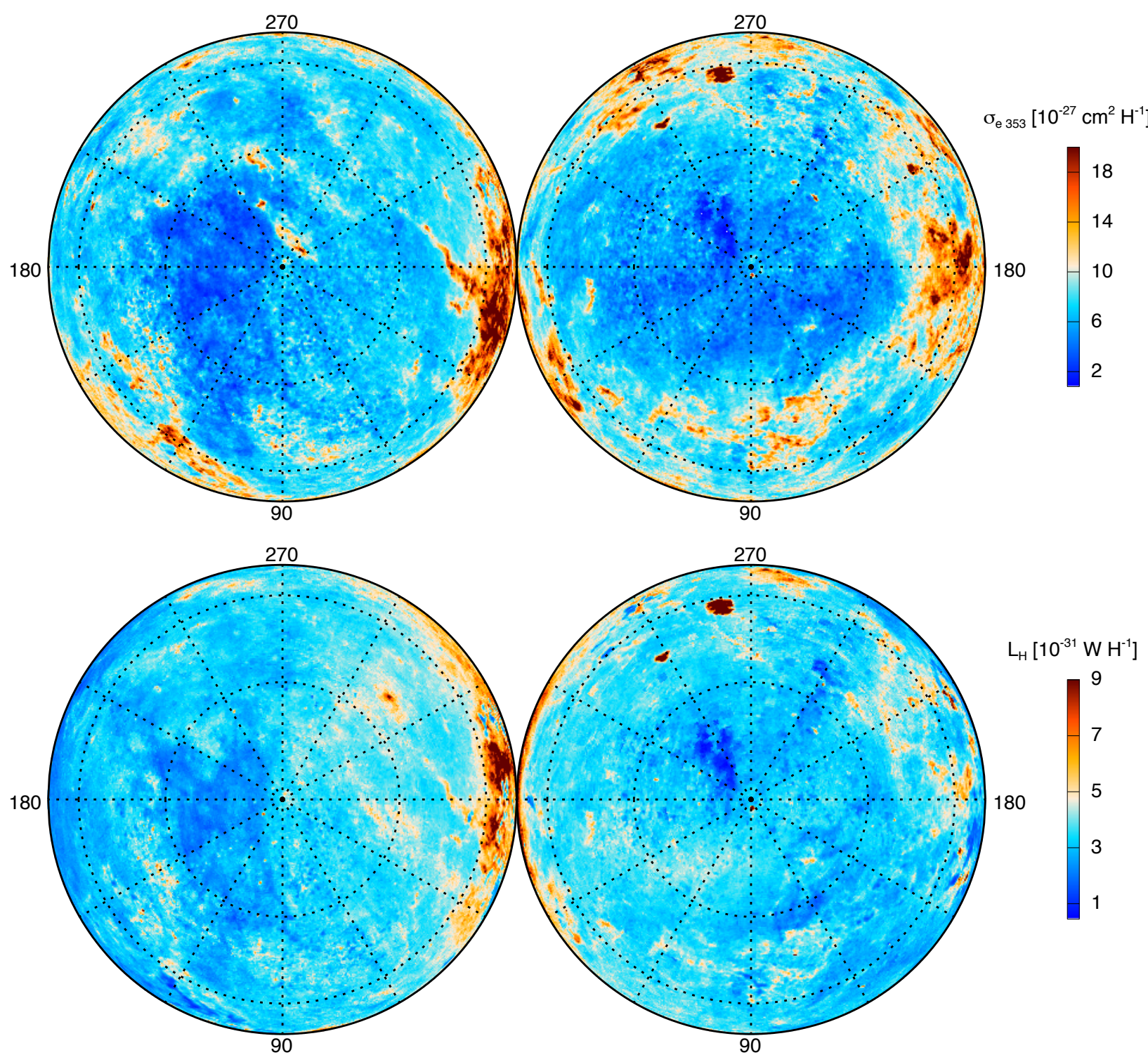

Fig. 18. Polar view of $\sigma_{\mathrm{e} 353}$ (upper) and $L_{\mathrm{H}}$ (lower).

To quantify the trends with column density, Fig. 20 shows the mean and standard deviation of $\sigma_{\mathrm{e} 353}$ and $L_{\mathrm{H}}$ in bins of $N_{\mathrm{H}}$ equally spaced in $\log$. The results for $X_{\mathrm{CO}}=[1,2,3] \times$ $10^{20} \mathrm{H}_{2} \mathrm{~cm}^{-2} \mathrm{~K}^{-1} \mathrm{~km}^{-1} \mathrm{~s}$ are shown.

\subsection{The strength of the ISRF}

In thermal equilibrium, $\mathcal{R}$ is equal to the amount of light absorbed by dust (see Eq. (11)). In normalized form, $L_{\mathrm{H}} \propto U \overline{\sigma_{\mathrm{a}}}$. Also from Eq. (10) for $\mathcal{R}$ evaluated from emission, $L_{\mathrm{H}}$ depends on $T_{\mathrm{obs}}, \beta_{\mathrm{obs}}$, and $\sigma_{\mathrm{e} 353}$. Therefore, under the constraint of thermal equilibrium, the measured $\mathcal{R}$ and $L_{\mathrm{H}}$ provide insight into not only $U$ and $\overline{\sigma_{\mathrm{a}}}$ relating to absorption, but also their relationship to the SED parameters for emission.

Under the hypothesis of a constant dust-to-gas ratio, constant dust absorption cross section, and constant shape of the ISRF spectrum, i.e., constant $\overline{\sigma_{\mathrm{a}}}$, the all-sky map of $L_{\mathrm{H}}$ provides a way to trace the spatial structure of the radiation field $U$ over the whole sky quite directly. The large-scale structure of this map is similar to the map of $C^{+} / N_{\mathrm{HI}}$, obtained from lower-resolution data by Bennett et al. (1994), that also traces $U$.

\subsubsection{High latitudes}

At high latitudes, best seen in the polar maps, $L_{\mathrm{H}}$ is fairly uniform, much more so than the opacity, as quantified by the relative fractional size of their standard deviations (Table 4). This can also be seen over the low column-density range of Fig. 20, lower, where $L_{\mathrm{H}}$ is constant up to $N_{\mathrm{HI}}=5.5 \times 10^{20} \mathrm{~cm}^{-2}$ which is a threshold criterion in the $|b|>15^{\circ}$ mask. Even in that mask the standard deviation of $L_{\mathrm{H}}$ is less than $20 \%$. We also note again that this column density is below that for which significant $\mathrm{H}_{2}$ is seen in the diffuse ISM (Gillmon et al. 2006; Wakker 2006; Rachford et al. 2002, 2009). Furthermore, there is unlikely to be local attenuation of the ISRF at such low column densities ${ }^{13}$. Short of a conspiracy among the several factors affecting $L_{\mathrm{H}}$, this suggests that each of the factors is fairly uniform in the

\footnotetext{
${ }^{13}$ Because the ISRF illumination is not just from along our line of sight, it is difficult to quantify the attenuation just from the observed column density. The total line of sight extinction is $A_{V}=0.053 N_{\mathrm{H}} /\left(10^{20} \mathrm{~cm}^{-2}\right)$ in largely atomic regions (see discussion and references in Martin et al. 2012), and so roughly half of this amount to the centre of a structure.
} 

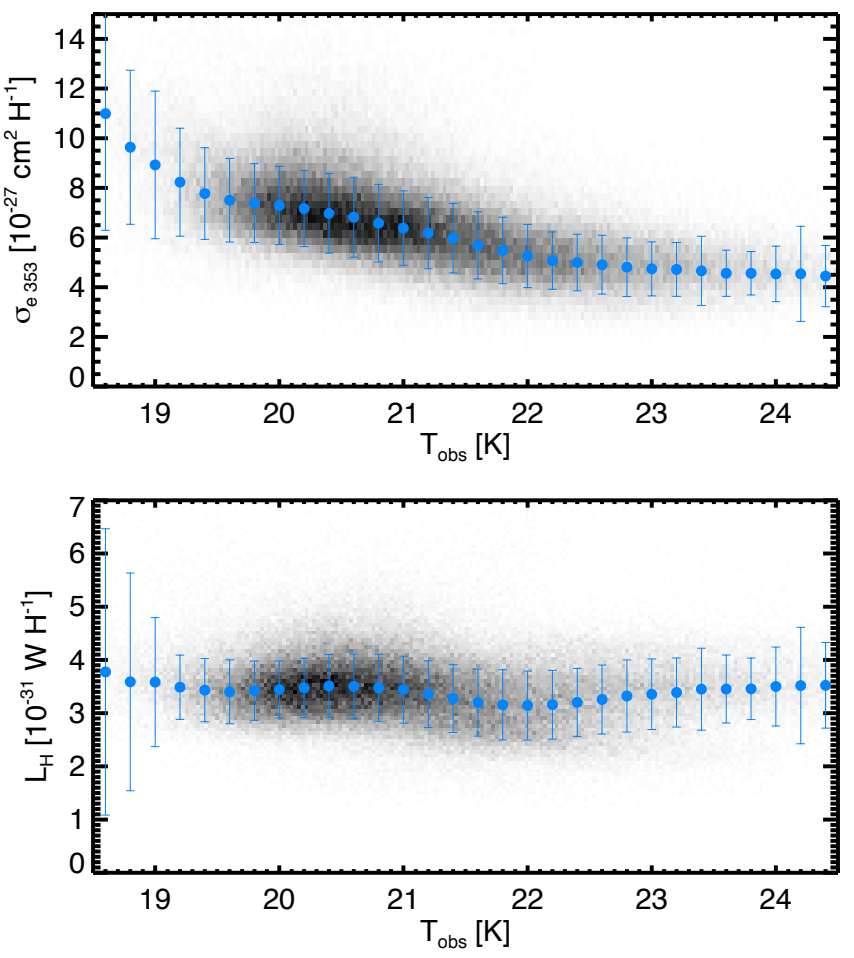

Fig. 19. Variation of $\sigma_{\mathrm{e} 353}$ (upper) and $L_{\mathrm{H}}$ (lower) as a function of $T_{\mathrm{obs}}$ in the diffuse ISM. Data used were smoothed to $30^{\prime}$, the angular resolution of the HI data. Only pixels where $1 \times 10^{20}<N_{\mathrm{HI}}<2.5 \times$ $10^{20} \mathrm{~cm}^{-2}$ were selected. The greyscale shows the point density in the two-dimensional histogram while the blue points indicate the average and standard deviation of $L_{\mathrm{H}}$ and $\sigma_{\mathrm{e} 353}$ in bins of $T_{\mathrm{obs}}$.

diffuse atomic high-latitude ISM, up to column densities of at least $5 \times 10^{20} \mathrm{~cm}^{-2}$.

There is a relatively flat trend of $L_{\mathrm{H}}$ with respect to $T_{\mathrm{obs}}$ in Fig. 19, lower, which is for moderate column densities $1 \times 10^{20}<$ $N_{\mathrm{HI}}<2.5 \times 10^{20} \mathrm{~cm}^{-2}$. This uniformity is in contrast to that for $\sigma_{\mathrm{e} 353}$ in the same figure which is anticorrelated with $T_{\mathrm{obs}}$ along a locus of constant $L_{\mathrm{H}}$, a phenomenon also reported by Planck Collaboration XXIV (2011) and Planck Collaboration Int. XVII (2014). There is also a striking difference between the polar maps of $L_{\mathrm{H}}$ and of $T_{\mathrm{obs}}$. This demonstrates that $T_{\mathrm{obs}}$ is not a simple tracer of $U$ as is often assumed. In particular, it suggests that the increase of $T_{\text {obs }}$ observed toward the Galactic pole is not a direct result of an increase of $U$. One interpretation, put forward by Martin et al. (2012), is that grains in different regions of the diffuse ISM retain the effects of different past histories of evolution, e.g., through aggregation and fragmentation, even though the density and timescale argue against present in situ evolution by such processes (Planck Collaboration XXIV 2011). Alternatively, Planck Collaboration Int. XVII (2014) review arguments that grain evolution could be occurring in situ due to UV radiative processing or exposure to cosmic rays. In either case, $T_{\text {obs }}$ would be a response to and tracer of variations in dust properties (grain structure, size distribution, material changes) rather than variations in the strength of the ISRF ${ }^{14}$. A corollary is that $\mathcal{R}$ could be a better alternative to $\tau_{353}$ as a tracer of $N_{\mathrm{H}}$, at least at high latitudes.

\footnotetext{
14 This result was shown by Planck Collaboration XXIV (2011) to be robust against $\beta-T$ anticorrelation effects. These authors reported even stronger variations of $T_{\mathrm{obs}}$ at constant $L_{\mathrm{H}}$ using a fit with a fixed $\beta_{\mathrm{obs}}$.
}
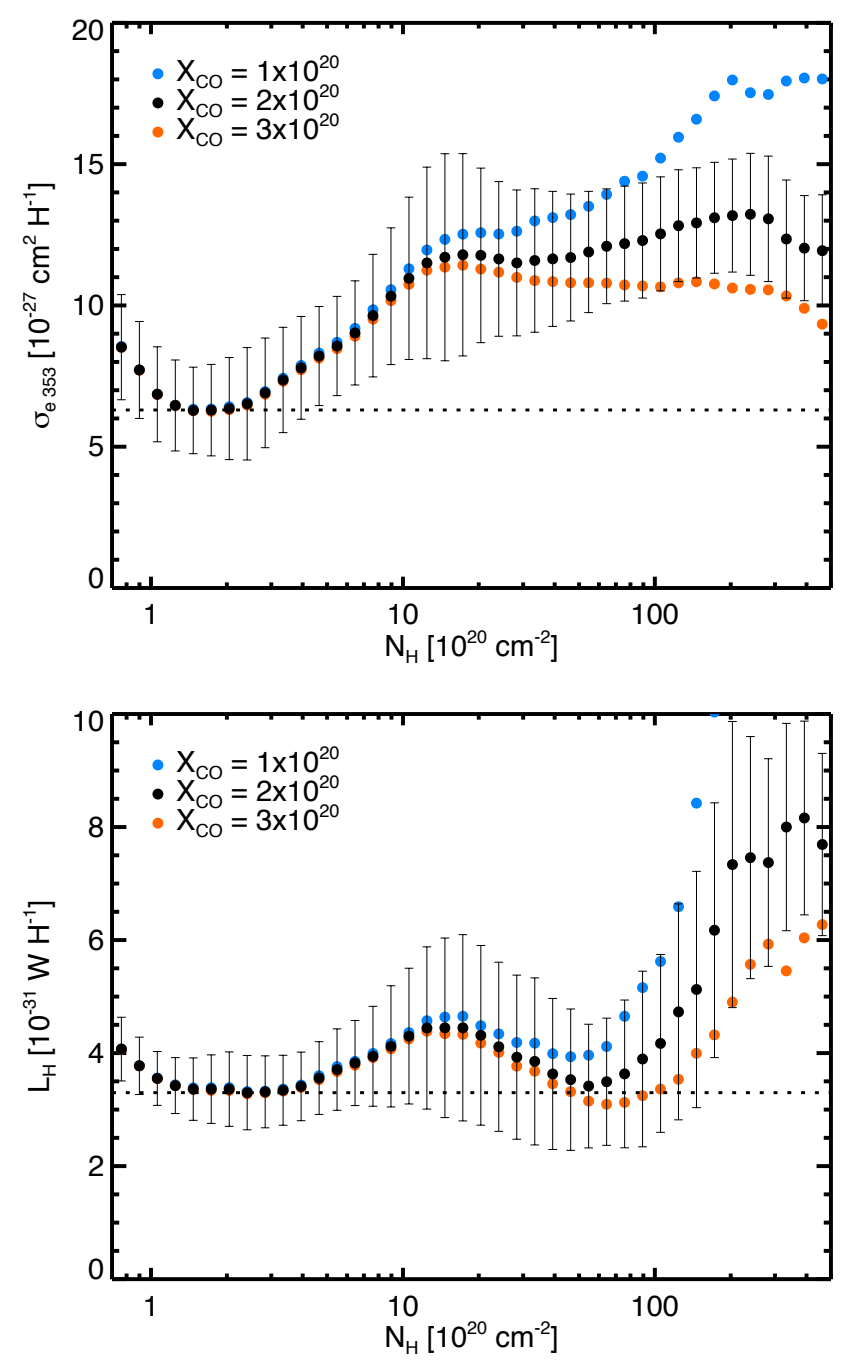

Fig. 20. $\sigma_{\mathrm{e} 353}$ (upper) and $L_{\mathrm{H}}$ (lower) vs. $N_{\mathrm{H}}$ computed at $30^{\prime}$ resolution from Eq. (13) with three values of $X_{\mathrm{CO}}$ : $[1,2,3] \times$ $10^{20} \mathrm{H}_{2} \mathrm{~cm}^{-2} \mathrm{~K}^{-1} \mathrm{~km}^{-1} \mathrm{~s}$. The mean values of $\sigma_{\mathrm{e} 353}$ and $L_{\mathrm{H}}$ were computed in bins of $N_{\mathrm{H}}$ equally spaced in log; the error bar is the standard deviation, shown only for $X_{\mathrm{CO}}=2 \times 10^{20} \mathrm{H}_{2} \mathrm{~cm}^{-2} \mathrm{~K}^{-1} \mathrm{~km}^{-1} \mathrm{~s}$. The horizontal dotted line indicates the average values found in the low $N_{\mathrm{HI}}$ mask.

There is a remarkable region near the south Galactic pole with abnormally low $L_{\mathrm{H}}$, but it does not show up in the $\mathcal{R}$ map. Planck Collaboration Int. XVII (2014) argue that it arises because of gas in the Magellanic Stream (MS) that has Galactic velocities and so is counted in $N_{\mathrm{H}}$ while at the same time the dust abundance and dust emission in the low-metalicity MS is very low. Planck Collaboration XXIV (2011) have shown that high velocity clouds (HVC) have relatively low emissivities, which suggests more generally that anomalously low $L_{\mathrm{H}}$ is an interesting diagnostic of HVC-like material that does not have a distinctive HVC velocity. But it is not an argument against using $\mathcal{R}$ as a tracer of column density.

However, comparison of the polar maps of IVC (Fig. 2, lower) and $L_{\mathrm{H}}$ shows a correlation of IVC column density with slightly lower $L_{\mathrm{H}}$ over widespread regions. Our interpretation follows Planck Collaboration XXIV (2011) who studied the emissivity of LVC and IVC gas separately and concluded that IVC is Galactic gas that often, though not always, has a lower $L_{\mathrm{H}}$ because dust has been partially destroyed; the dust-to-gas ratio 


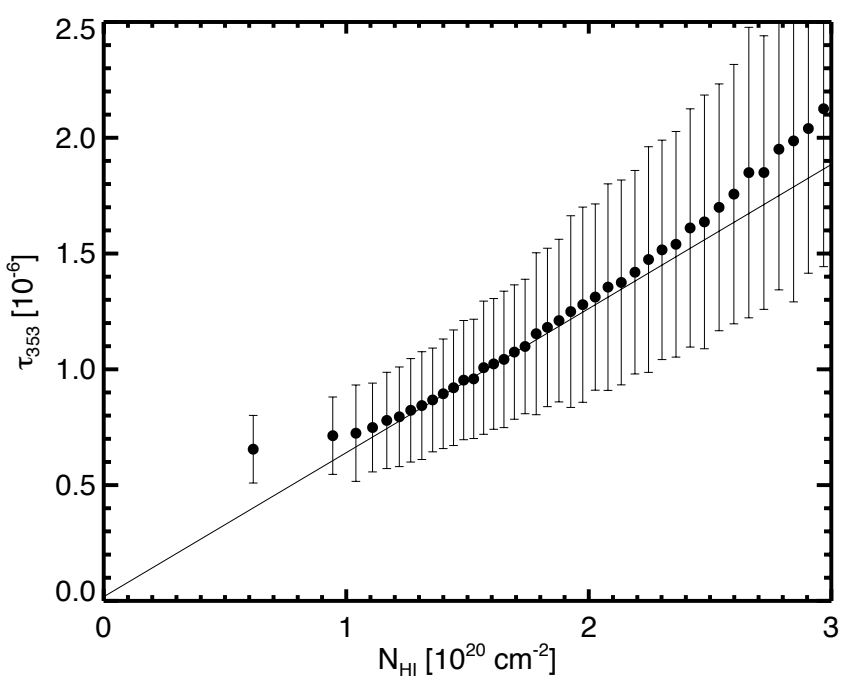

Fig. 21. $\tau_{353}$ as a function of $N_{\mathrm{HI}}$ estimated at $30^{\prime}$ resolution. Each point and its associated bar is the mean and standard deviation of $\tau_{353}$ in bins of $N_{\mathrm{HI}}$. The solid line is the linear regression fit using pixels for which $1.2<N_{\mathrm{HI}}<2.5 \times 10^{20} \mathrm{~cm}^{-2}$. Its parametrization is $\tau_{353}=$ $6.3 \pm 0.1 \times 10^{-27} N_{\mathrm{HI}}-0.02 \times 10^{-6}$.

is lower in that component of gas along the line of sight. The amount of such IVC gas would be underpredicted by $\mathcal{R}^{15}$.

\subsubsection{Intermediate to low latitudes}

The all-sky map also shows that $L_{\mathrm{H}}$ is not constant, thus strong evidence against using $\mathcal{R}$ everywhere as a tracer of column density. For example, the increase of $L_{\mathrm{H}}$ in the inner Galaxy instead implies an increase of the radiation field strength there, by a factor about three compared to the local ISM. The all-sky $L_{\mathrm{H}}$ map also suggests that the ISRF is generally weaker in the outer Galaxy, as expected. Note also that an increase (decrease) in $L_{\mathrm{H}}$ can also be the result of an increase (decrease) in the dust-to-gas ratio accompanying a higher (lower) metallicity.

More localized regions of high $L_{\mathrm{H}}$ are present too: active star formation sites like Cyg X where local sources significantly enhance the IRSF illuminating the dust.

There are localized decreases in $L_{\mathrm{H}}$ as well, coincident with recognizable intermediate-latitude molecular clouds. Our interpretation is that this is a result of a lower ISRF because of attenuation, lowering the energy absorbed by dust within the clouds and hence available to be emitted. In these regions too, $\mathcal{R}$ would be compromised as a quantitative linear tracer of column density (see discussion below relating to the Taurus cloud in Fig. 23).

All of these factors contribute to the complicated change of the mean and standard deviation of $L_{\mathrm{H}}$ in Fig. 20, lower, at $N_{\mathrm{HI}}>$ $5.5 \times 10^{20} \mathrm{~cm}^{-2}$, the part of the sky that is the complement to the $|b|>15^{\circ}$ mask.

\subsection{Dust opacity from the diffuse ISM to molecular clouds}

The maps of $\sigma_{\mathrm{e} 353}$ in the upper panels of Figs. 17 and 18 reveal variations of the opacity over the sky, variations that are spatially coherent. In the polar plots, the anticorrelation of $\sigma_{\mathrm{e}} 353$ with $T_{\mathrm{obs}}$ is apparent, the same as summarized in Fig. 19 (upper).

\footnotetext{
${ }^{15}$ Note that pixels with strong IVC were excluded from the low $N_{\mathrm{HI}}$ mask used to establish the zero points of the intensity maps.
}

Because of the $\beta_{\mathrm{obs}}-T_{\mathrm{obs}}$ anticorrelation discussed in Sect. 4.2, also seen clearly in the polar maps, there is a correlation of $\sigma_{\mathrm{e} 353}$ and $\beta_{\text {obs }}$ as well. Thus at high latitude $\tau_{353}$ is not a reliable measure of $N_{\mathrm{HI}}$.

The general increase of $\sigma_{\mathrm{e} 353}$ by almost a factor of three toward higher column density can be followed on the all-sky map down to intermediate latitudes, to known molecular clouds (e.g., Taurus, Orion, $\rho$ Ophiuchi). These tend to have lower $T_{\text {obs }}$ and higher $\beta_{\mathrm{obs}}{ }^{16}$.

Figure 20, upper, shows the dependence of $\sigma_{\mathrm{e} 353}$ on $N_{\mathrm{HI}}$. There is a small range at low $N_{H}$ over which $\sigma_{\mathrm{e} 353}$ is at a minimum and roughly constant. But as shown in Fig. 21, where the slope of a fit of $\tau_{353}$ vs. $N_{\mathrm{HI}}$ over the same range of $N_{\mathrm{HI}}$ corresponds to the same $\sigma_{\mathrm{e} 353}$, the non-linear increase of $\tau_{353}$ (and $\left.\sigma_{\mathrm{e} 353}\right)$ with $N_{\mathrm{HI}}$ sets in at a rather low column density. This in a range where as discussed above $L_{\mathrm{H}}$ is constant and there is no significant molecular hydrogen or H I self-absorption. Thus the increase of $\sigma_{\mathrm{e} 353}$ is real and not a reflex of unaccounted dark gas. Again, this compromises $\tau_{353}$ as a measure of $N_{\mathrm{HI}}$ in the diffuse ISM.

The opacity continues to increase over the range $3 \times$ $10^{20}<N_{\mathrm{H}}<1 \times 10^{21} \mathrm{~cm}^{-2}$ reaching a plateau thereafter, with a dependency on the choice of $X_{\mathrm{CO}}$ since the gas is predominantly molecular there. The choice of $X_{\mathrm{CO}}=2.0 \times$ $10^{20} \mathrm{H}_{2} \mathrm{~cm}^{-2} \mathrm{~K}^{-1} \mathrm{~km}^{-1} \mathrm{~s}$ recommended by Bolatto et al. (2013), results in a flat plateau at about twice the value in the diffuse ISM (dotted line).

\subsection{Dust at the lowest column densities}

At the lowest column densities $\left(N_{\mathrm{H}}<1 \times 10^{20} \mathrm{~cm}^{-2}\right)$ we note an increase of $\sigma_{\mathrm{e} 353}$ and $L_{\mathrm{H}}$ (Fig. 20). This effect is also seen directly in the correlation of $I_{v}$ vs. $N_{\mathrm{HI}}$ in Fig. 5 where all 857 and $3000 \mathrm{GHz}$ data points fall above the correlation for $N_{\mathrm{HI}}<$ $1.0 \times 10^{20} \mathrm{~cm}^{-2}$. It is also the case at 545 and $353 \mathrm{GHz}$ and it thus propagates into the map of $\tau_{353}$ and $\mathcal{R}$. We checked that this effect is independent of the removal of the zodiacal emission. It is also present in $E(B-V) / N_{\mathrm{H}}$ using the $E(B-V)$ map of Schlegel et al. (1998) which is based on DIRBE.

Using correlation studies, Planck Collaboration XXIV (2011) showed that $\mathrm{HI}$ is a reliable tracer of dust up to at least $N_{\mathrm{HI}}=2 \times 10^{20} \mathrm{~cm}^{-2}$, or as discussed in Sect. 5.2.1 $\mathcal{R}$ is a good tracer of $N_{\mathrm{H}}$ to somewhat higher column densities. This suggests that the excess opacity at the lowest $N_{\mathrm{HI}}$ seen here in this pixel by pixel analysis is the signature of dust associated with the warm ionized medium (WIM), i.e., interstellar dust that is mixed with ionized hydrogen, $\mathrm{H}+$, that is not traced by $\mathrm{HI}$ emission.

Assuming that dust in the WIM has a similar $L_{\mathrm{H}}$ as in the $\mathrm{HI}$, the WIM gas column density needed to explain the rise at low $N_{\mathrm{HI}_{\mathrm{I}}}$ is $N_{\mathrm{H}+} \sim 3 \times 10^{19} \mathrm{~cm}^{-217}$. However, a constant value of $N_{\mathrm{H}+}$ cannot explain the shape of the rise of $L_{\mathrm{H}}$. The rise is more compatible with $N_{\mathrm{H}+}+N_{\mathrm{HI}} \approx 1.1 \times 10^{20} \mathrm{~cm}^{-2}$ suggestive of an

16 The Magellanic Clouds have an opacity almost five times that in the diffuse ISM, despite the low metallicity. But $\beta_{\text {obs }}$ is unusually low, pointing to a mixture of conditions within the beam and so an SED that is unlikely characterized by a single temperature. $T_{\mathrm{obs}}$ is relatively high and dust in ionized gas could be contributing.

17 This result seems compatible with Lagache et al. $(1999,2000)$ who showed that dust in the WIM has similar emissivity to dust in the WNM but a slightly higher temperature. These authors also concluded that about $25 \%$ of the dust emission in the diffuse ISM is associated with the WIM, uncorrelated with $\mathrm{HI}$, a value which corresponds well with what is seen here for $N_{\mathrm{HI}}<1.2 \times 10^{20} \mathrm{~cm}^{-2}$. 
increase of the ionization fraction of WNM toward lower $N_{\mathrm{HI}}$. The apparent extra dust emission seen here would then come from diffuse regions where $\mathrm{HI}$ is partly ionized.

\subsection{Discussion}

The comparison of dust emission and gas column density reveals an increase of dust opacity of a factor about two from the diffuse ISM to molecular clouds (Fig. 20, upper). In the translucent transition region $\left(3 \times 10^{20}<N_{\mathrm{H}}<2 \times 10^{21} \mathrm{~cm}^{-2}\right) \mathrm{H}_{2}$ might start rising in importance before $\mathrm{CO}$ and self-absorption could begin to affect the $21 \mathrm{~cm}$ line emission. These effects are difficult to quantify from the present data but they cannot be responsible for the systematic difference in $\sigma_{\mathrm{e} 353}$ observed between the $\mathrm{HI}$ dominated and $\mathrm{CO}$ dominated regimes. The presence of "dark gas", whether from $\mathrm{H}_{2}$ not traced by $\mathrm{CO}$ or from $21 \mathrm{~cm}$ self-absorption, would simply flatten the rising profile of $\sigma_{\mathrm{e} 353}$ vs. $N_{\mathrm{H}}$, reaching the plateau somewhat later. The fact that at $N_{\mathrm{H}} \sim 6 \times 10^{21} \mathrm{~cm}^{-2}$, a column density where CO is thought to be a reliable tracer of $N_{\mathrm{H}}, L_{\mathrm{H}}$ dips to the diffuse ISM value while $\sigma_{\mathrm{e} 353}$ remains at the plateau value (Fig. 20) is also consistent with an increased dust opacity in denser regions.

As discussed further in Sect. 6.2, this increase of $\sigma_{\mathrm{e} 353}$ in denser regions is also seen when $N_{\mathrm{H}}$ is estimated using nearinfrared colour excess or star counts (Arce \& Goodman 1999; Cambrésy et al. 2001; Stepnik et al. 2003; Planck Collaboration XXV 2011; Martin et al. 2012; Roy et al. 2013). It is generally accompanied by a decrease of $T_{\mathrm{obs}}$, which is quite challenging to explain just with radiative transfer effects (Ysard et al. 2012). When the gas has become dense, an increased $\sigma_{\mathrm{e} 353}$ might be attributed to an increase of dust emissivity related to dust aggregation/coagulation (Ossenkopf \& Henning 1994; Ormel et al. 2011; Köhler et al. 2012) ${ }^{18}$. However, we have seen opacity changes in the diffuse high-latitude ISM as well that need alternative interpretation if the evolution is in situ (Sect. 5.2.1).

Because $\mathcal{R}$ is less affected by the CIBA and because of its correlation with $N_{\mathrm{H}}$ over a larger range in column density (see $L_{\mathrm{H}}$ in Fig. 20, lower), we conclude that $\mathcal{R}$ is preferred over $\tau_{353}$ as a tracer of column density in the high-latitude diffuse ISM, at least for $N_{\mathrm{H}}<5 \times 10^{20} \mathrm{~cm}^{-2}$. However, this preference does not hold in molecular clouds and star forming regions where $\mathcal{R}$ traces not only the column density but also variations of the radiation field strength due to attenuation and/or local sources of heating photons. In such regions, $\tau_{353}$ is the preferred tracer of column density, to the extent that $\sigma_{\mathrm{e} 353}$ is constant there ${ }^{19}$. This is supported empirically by the good correlation with the colour excess $E\left(J-K_{\mathrm{s}}\right)$ discussed below in Sect. 6.2. However, finding the absolute, rather than relative, column density depends on proper calibration of the opacity $\sigma_{\mathrm{e} v}$, which appears to vary with column density and be larger in these regions. Furthermore, caution is advised because the opacity changes from diffuse to dense regions, which might occur over the range of column densities encountered in the region being analysed.

\footnotetext{
${ }_{18}$ With the increase of gas density, smaller grains stick on the surface of bigger ones, modifying their structure to a more open one, resulting in an increase of emissivity. Being more emissive, the grains cool more efficiently and are therefore colder.

${ }^{19}$ Using higher resolution Herschel data to probe opacity to high column densities, Roy et al. (2013) found evidence for a non-linear increase of $\tau_{353}$ with $N_{\mathrm{H}}$.
}

\section{Dust emission in relation to extinction}

A quantity often used to estimate interstellar column density is visible or near-infrared extinction measured along lines of sight to point sources: stars, globular clusters, galaxies, or quasars. It has been established long ago that there exists a correlation between gas and dust column densities, in particular through the comparison of $21 \mathrm{~cm}$ emission and visible extinction (e.g., Lilley $1955)$. The linear relationship between $N_{\mathrm{HI}}$ and $E(B-V)$ was established in the 1970s (Savage \& Jenkins 1972; Knapp \& Kerr 1974; Ryter et al. 1975; Bohlin et al. 1978) ${ }^{20}$. Knapp \& Kerr (1974) advocated using $21 \mathrm{~cm}$ observations of $N_{\mathrm{H} \text { I }}$ as a proxy for extinction and this correlation, especially as calibrated in the diffuse ISM using measurements on extragalactic objects, has been key to correct extragalactic observations for Galactic reddening.

We have seen in Sect. 5 how the amount of dust emission is, not surprisingly, also correlated with $N_{\mathrm{H}}$. However, because dust is the agent in both extinction and emission, we make a direct comparison of these observables rather than using $N_{\mathrm{H}}$ as an intermediary. An important example of this direct approach is the proposal by Schlegel et al. (1998) to use dust optical depth obtained from FIR emission (IRAS and DIRBE), rather than $\mathrm{HI}$, to estimate reddening $\left(E(B-V)_{\mathrm{SFD}}\right)$, through a correlation calibrated on reddening measurements of galaxies. Such an approach is pursued in Sect. 6.1. For higher column density lines of sight, we compared dust emission to colour excess measurements based on 2MASS stellar photometry ${ }^{21}$.

\subsection{Correlation with $E(B-V)$ from quasars}

Here, based on the Planck dust emission, we develop a map of $E(B-V)$ applicable to the diffuse ISM at high Galactic latitude. In the years since the work of Schlegel et al. (1998), many models have been put forward self-consistently describing dust emission and extinction (e.g., Draine \& Li 2007; Compiègne et al. 2011) and they could be used to convert emission to extinction. However, to be independent of any assumption about dust properties, we decided to remain with an empirical approach. We estimate the conversion factor to $E(B-V)$ using measurements of extinction of extragalactic objects rather than stars to avoid potential biases due to background dust emission.

In particular we estimated $E(B-V)$ using Sloan Digital Sky Survey (SDSS) measurements of quasars. We used the final edition of the SDSS-II quasar catalogue (Schneider et al. 2010) based on the seventh SDSS data release (Abazajian et al. 2009). The catalogue contains 105783 objects spread over $8400 \mathrm{deg}^{2}$ mostly on the northern Galactic hemisphere. For each quasar, the observed magnitudes in bands $u, g, r, i$, and $z$ are given together with their uncertainties. All objects in this catalogue have highly reliable redshift estimates. We limited the sample to a subset of 53399 quasars at redshifts for which Ly $\alpha$ does not enter the SDSS filters. One benefit compared to the work of Schlegel et al. (1998) is the much larger number of objects. Another is that many studies based on SDSS data have shown that the shape of the extinction curve in the diffuse ISM is compatible with that

${ }^{20}$ Key information on dust is derived from this relationship, for example that dust contains only $1 \%$ of the mass of the ISM, and this relationship remains a important constraint for dust models (Draine \& Li 2007; Compiègne et al. 2011).

${ }^{21}$ The Two Micron All Sky Survey (Skrutskie et al. 2006) is a joint project of the University of Massachusetts and the Infrared Processing and Analysis Center/California Institute of Technology, funded by the National Aeronautics and Space Administration and the National Science Foundation. 
for stars from Fitzpatrick (1999) with $R_{V}=3.1$ (Jones et al. 2011; Schlafly \& Finkbeiner 2011; Mörtsell 2013), so that we can take advantage of all of the multi-colour measurements. The details of how we estimate $E(B-V)$ for each quasar are given in Appendix E.

The correlations of $E(B-V)$ with Planck $\mathcal{R}$ and $\tau_{353}$, from which point sources have been removed (Sect. 3.4), are shown in Fig. $22^{22}$. Each is strongly correlated: $E(B-V) / \mathcal{R}=(5.40 \pm$ $0.09) \times 10^{5}$ and $E(B-V) / \tau_{353}=(1.49 \pm 0.03) \times 10^{4}$. The fractional uncertainty of the slope of the correlation with $\mathcal{R}$ is about $20 \%$ lower than that for $\tau_{353}$. This is not unexpected, both from our discussion of Fig. 13 and, in Sect. 5.2.1, of the factors that influence $\tau_{353}$ but not $\mathcal{R}$. This leads us to prefer the solution based on $\mathcal{R}, E(B-V)_{\mathcal{R}}$, to that based on $\tau_{353}, E(B-V)_{\tau 353}$, for the low column density regions of the sky (see also Sect. 5.5).

The product of $E(B-V) / \mathcal{R}$ and the diffuse ISM estimates of $L_{\mathrm{H}}$ gives the ratio $E(B-V) / N_{\mathrm{H}}=(1.42-1.46) \times 10^{-22} \mathrm{mag} \mathrm{cm}^{2}, \mathrm{a}$ factor just $0.82-0.85$ lower than that measured using background stars for lines of sight with considerably larger $E(B-V)$ (Bohlin et al. 1978; Rachford et al. 2009). Given all the potential for differences, this agreement is remarkable. On the other hand, the same ratio found using $E(B-V) / \tau_{353}$ and the low value of $\sigma_{\mathrm{e} 353}$ in Figs. 20, upper, and 21 and in Table 4 results in a factor $0.55-$ 0.60 lower. Using the $E(B-V)$ map derived from dust emission $\tau_{3000}$ by Schlegel et al. (1998), Liszt (2014) also found a lower ratio, by a factor 0.7 , for lines of sight with $E(B-V)<0.1$.

\subsection{Comparing to $E\left(J-K_{\mathrm{s}}\right)$ from star colours in molecular clouds}

We have investigated how to estimate $E(B-V)$ for nearby molecular clouds from Planck dust emission. A point of comparison for such regions is mapping of colour excesses $E(J-H)$ and $E\left(H-K_{\mathrm{s}}\right)$, or their sum $E\left(J-K_{\mathrm{s}}\right)$, based on stellar colours (e.g., Goodman et al. 2009). Here we use maps produced with the AvMAP technique and based on colour excesses from the 2MASS data base (Schneider et al. 2011). Similar maps can be obtained with the NICER and NICEST techniques (e.g., Lombardi et al. 2011). The effective angular resolution of the 2MASS extinction maps used here is a few minutes of arc (see, e.g., Roy et al. 2013), close to that of Planck.

Although the optical extinction is rarely directly measured in the same high column density regions (Martin et al. 2012; Roy et al. 2013), these near-infrared colour excesses are usually expressed as $E(B-V)_{2 \text { MASS }}$ after conversion using an assumed shape of the extinction curve, that for stars with $R_{v}=3.1$. This conversion might be inappropriate, and even variable across a field, because of dust evolution affecting all of the colour excess ratios for a given column of dust. Nevertheless, it is still very interesting to compare the spatial details of the dust column density revealed by dust extinction and by dust emission, because each is affected by different systematic effects.

For the Taurus and $\rho$ Ophiuchi molecular clouds, Figs. 23 and 24 present a comparison of four different estimates of $E(B-$ $V): E(B-V)_{2 \mathrm{MASS}}, E(B-V)_{\tau 353}, E(B-V)_{\mathrm{SFD}}$, and $E(B-V)_{\mathcal{R}}$. The Pearson correlation coefficients of $E(B-V)_{2 \text { MASS }}$ with the three other maps are given in Table 5. The correlation of $E(B-$ $V)_{\tau 353}$ map with $E(B-V)_{2 \text { MASS }}$ is excellent (Pearson coefficient of 0.86 for Taurus and 0.95 for $\rho$ Ophiuchi); this agreement is

\footnotetext{
${ }^{22}$ Although not an explicit selection criterion, the range of $\tau_{353}$ sampled by the selected quasars corresponds the conditions in the low $N_{\mathrm{HI}}$ mask, Fig. 3, whose NDF for $\tau_{353}$ is shown in Fig. 6. The positions of the bins along the $x$-axes in Fig. 22 reflect this NDF.
}
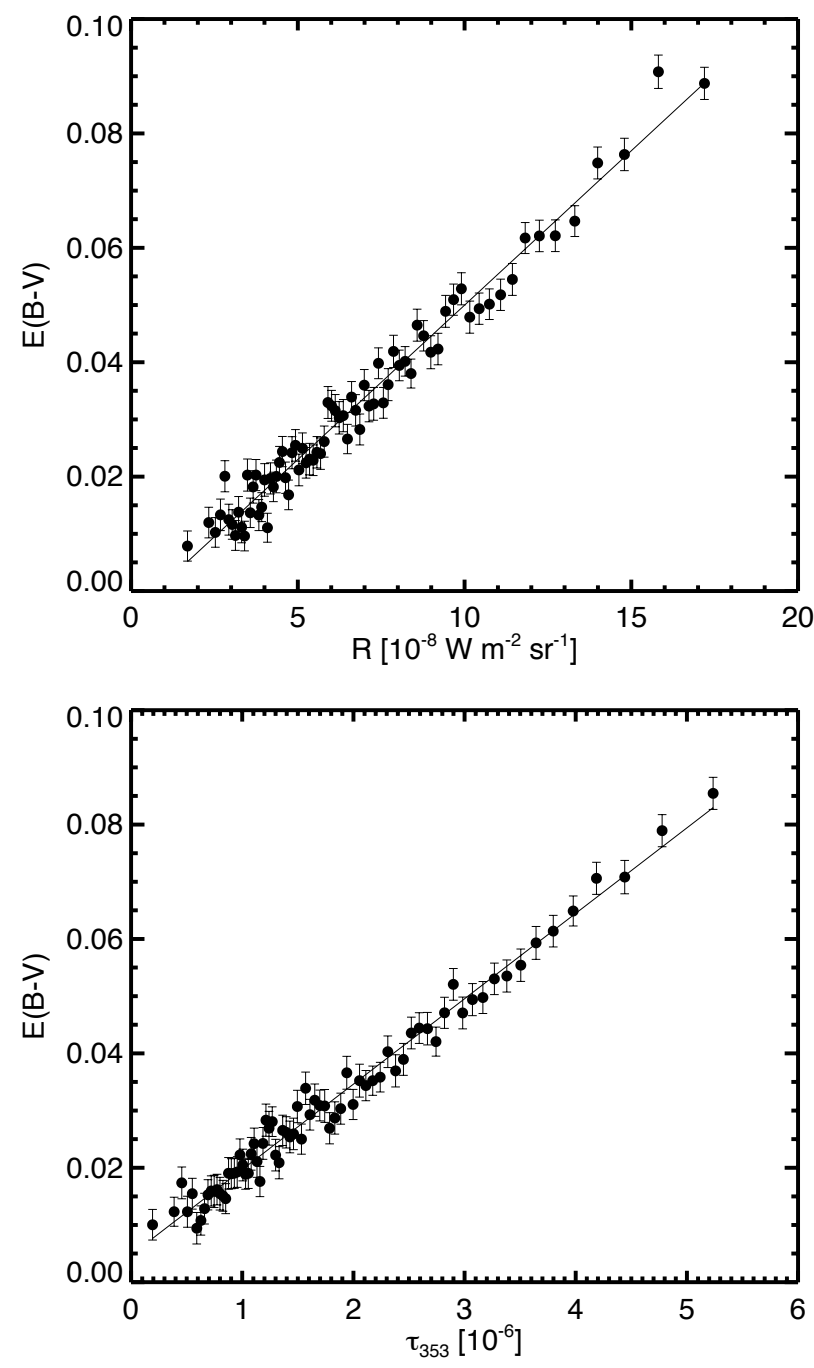

Fig. 22. $E(B-V)$ measured with quasars in the diffuse ISM at high Galactic latitude as a function of $\mathcal{R}$ (upper) and $\tau_{353}$ (lower). Each point is the average of $E(B-V)$ values in a bin of $\tau_{353}$ or $\mathcal{R}$. The bin size varies for each bin such that there is always the same number of samples per bin $(N=1000)$. The error bar is the standard deviation of $E(B-V)$ values in the bin, divided by $\sqrt{N}$. The solid line is the linear regression fit to the whole sample; $E(B-V) / \mathcal{R}=(5.40 \pm 0.09) \times 10^{5}$ and $E(B-V) / \tau_{353}=(1.49 \pm 0.03) \times 10^{4}$. In each case, the intercept is small $\left(-0.0040\right.$ for $\mathcal{R}$ and 0.0048 for $\left.\tau_{353}\right)$, in accordance with the fact that the zero levels of $E(B-V)$ (i.e., the intrinsic colours) and of the Planck and IRIS data were estimated using a correlation with $N_{\mathrm{HI}}$.

remarkable given that very different methods and data sets were used to build these two maps. However, notice how the brightest filamentary structures appear with more contrast in $E(B-V)_{\tau 353}$ than in $E(B-V)_{2 \mathrm{MASS}}$, a point to which we shall return.

The Schlegel et al. (1998) map $E(B-V)_{\mathrm{SFD}}$ was also produced from a dust optical depth map (at $3000 \mathrm{GHz}$ in that case; see Sect. 7.3 for details) and so the scale is similar. However, the correlation coefficient with $E(B-V)_{2 \mathrm{MASS}}$ is lower and it is clear that a lot of spatial detail is absent. This arises because they estimated $T_{\text {obs }}$ using the lower resolution DIRBE data, thus missing the dust temperature decrease at small scales that accompanies the increase of column density in molecular clouds, and so their map of optical depth which underlies $E(B-V)_{\mathrm{SFD}}$ has lower contrast as well as lower resolution. Being able to follow the small-scale variations of $T_{\text {obs }}$ appears to be essential to gauge properly the full structural details of the molecular clouds. This 

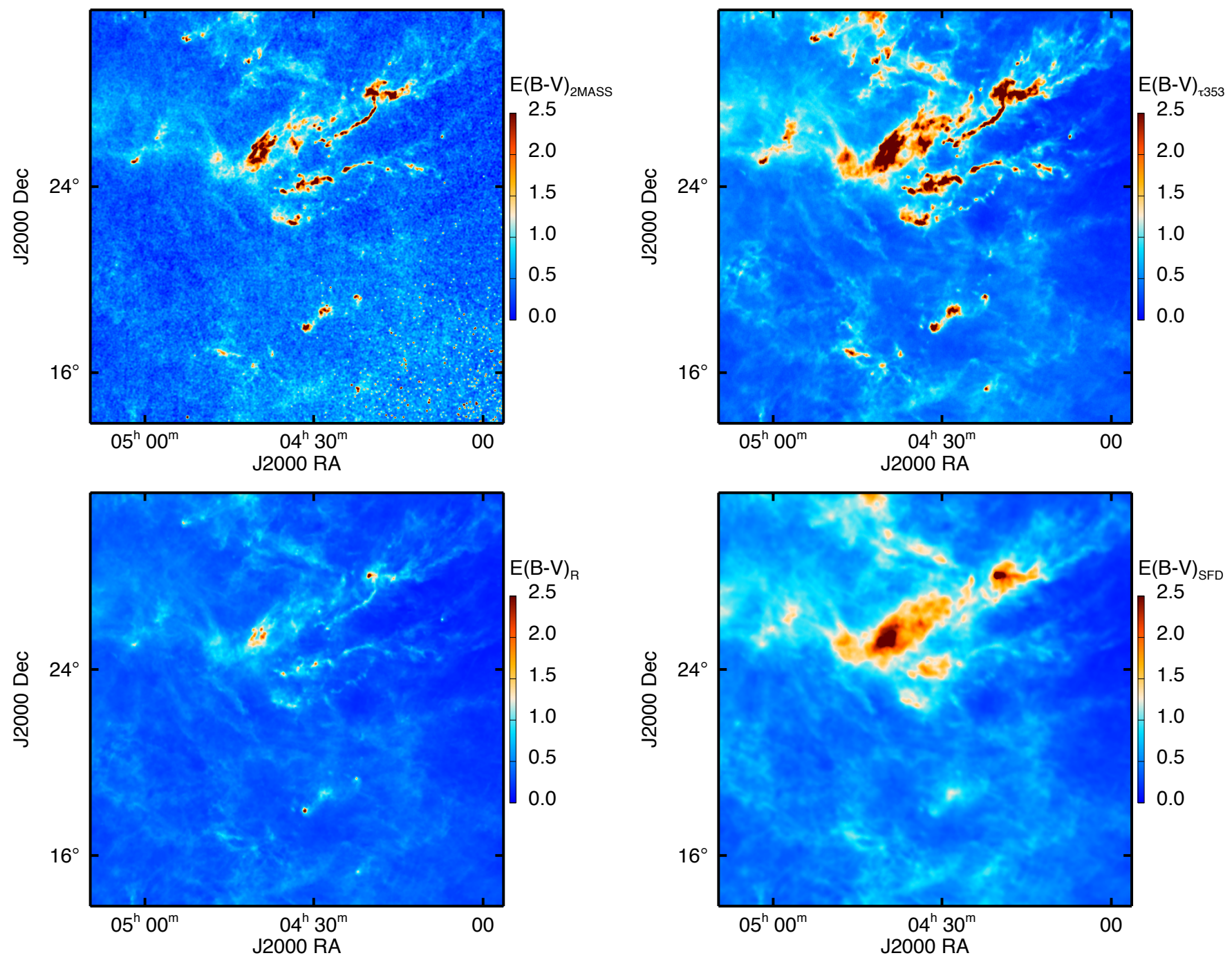

Fig. 23. Estimates of $E(B-V)$ in the Taurus molecular cloud. Clockwise from upper left: from 2MASS data, from $\tau_{353}$, from Schlegel et al. (1998), and from $\mathcal{R}$.

was revealed by previous studies (Cambrésy et al. 2001; Stepnik et al. 2003; Planck Collaboration XXV 2011), in particular using higher resolution Herschel data (Battersby et al. 2011; Roy et al. 2013), and is now confirmed and reinforced by our Planck analysis.

Although a lot of spatial detail is faithfully reproduced in $E(B-V)_{\mathcal{R}}$, thanks to the Planck resolution, the correlation with $E(B-V)_{2 \text { MAss }}$ is less good. Furthermore, the scale is off. In these denser regions of the ISM, the radiation field strength, and hence $\mathcal{R}$, varies locally due to attenuation and/or local production of photons. The first effect (attenuation) is apparent in Taurus where the densest and brightest filamentary structures appear with less contrast in $E(B-V)_{\mathcal{R}}$ than in $E(B-V)_{2 \text { MASS }}$, and these regions are, consistently, also colder. The case of $\rho$ Ophiuchi is different; because of active star formation, and thus local sources of heating photons, it is a photon-dominated region. The spatial structure of $\mathcal{R}$ is therefore visually different than that of $\tau_{353}$ or $E(B-V)_{2 \mathrm{MASS}}$, due to the spatial variation of the radiation field.

Opposite to what was found in the diffuse ISM, $\tau_{353}$ appears preferable to $\mathcal{R}$ as a tracer of column density, in this case $E(B-V)$. But as discussed next it is a complex situation warranting caution. A high correlation coefficient is an important criterion, but the scale and dynamic range are also important.
These effects can be appreciated by the quantification in Fig. 25 where the ratios $E(B-V)_{\tau 353} / E(B-V)_{2 \text { MASS }}$ and $E(B-V)_{\mathcal{R}} / E(B-V)_{2 \text { MASS }}$ are plotted as a function of $T_{\text {obs }}$.

In Taurus, $E(B-V)_{\tau 353}$ agrees with $E(B-V)_{2 \text { MASS }}$ over most of the map, although it is systematically high by typically $25 \%$. Arce \& Goodman (1999) reported a similar result in their study of $E(B-V)_{\mathrm{SFD}}$ in Taurus. The exception here is in the coldest parts of the cloud where $E(B-V)_{\tau 353} / E(B-V)_{2 \text { MASS }}$ increases even more. The systematic departure and spatial variations of $E(B-V)_{\tau 353} / E(B-V)_{2 \text { MASS }}$ appear to be the result of an increase in $\sigma_{\mathrm{e} 353}$ even in the relative diffuse parts of the map and even more in the coldest (densest) regions. The opacity changes at higher column densities are argued to be related to dust evolution (Planck Collaboration XXV 2011), and unless independently characterized these opacity changes compromise the interpretation of $E(B-V)_{\tau 353}$ as a quantitative measure of dust column density. This is a general concern for all column densities derived from FIR and submillimetre optical depth.

In Taurus, $E(B-V)_{\mathcal{R}}$ also agrees with $E(B-V)_{2 \text { MASs }}$ over large parts of the map, although it is slightly low systematically (Fig. 25). In the densest regions, which are cold because of attenuation of the ISRF, $L_{\mathrm{H}}$ is depressed even further and 

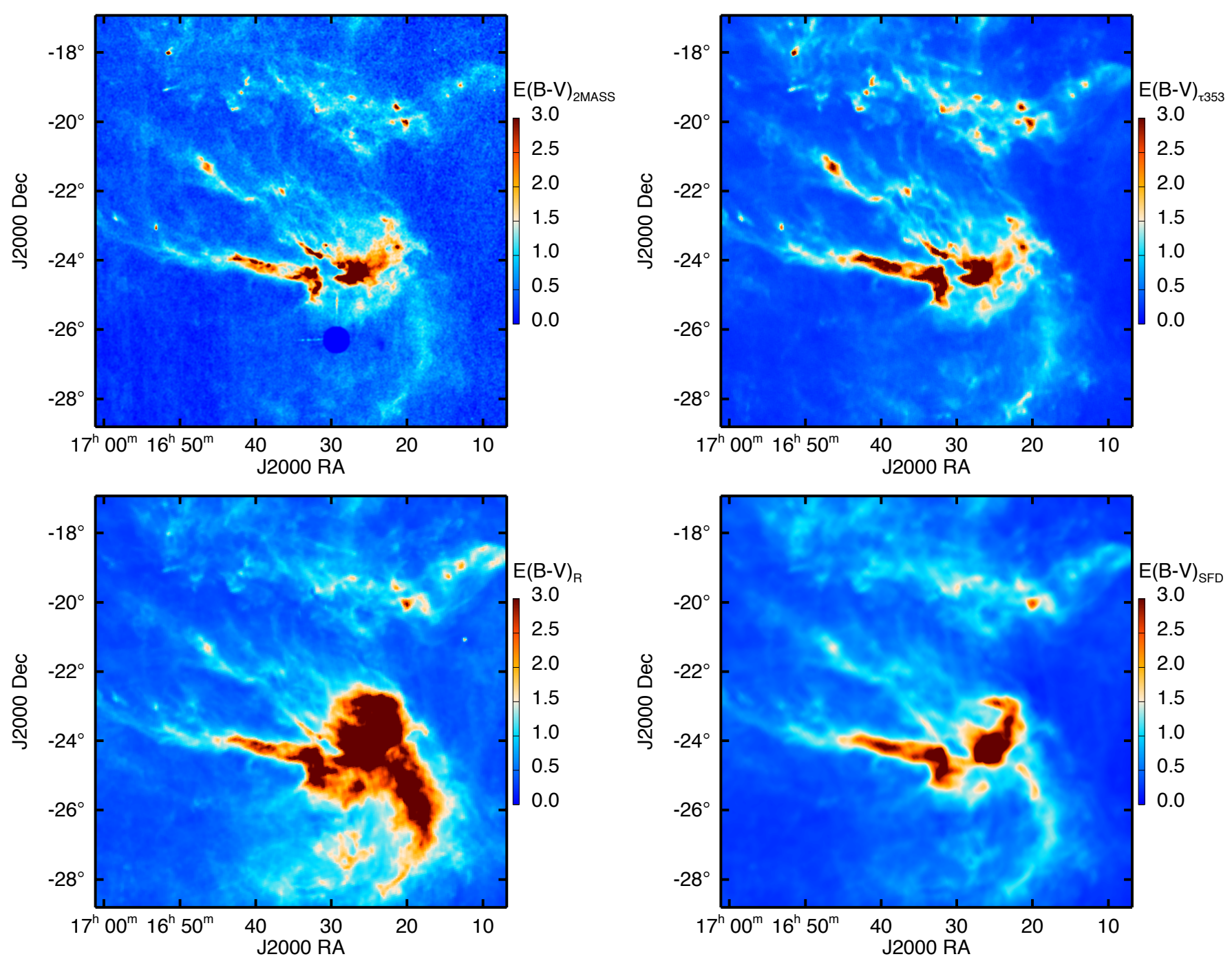

Fig. 24. Estimates of $E(B-V)$ in the $\rho$ Ophiuchi molecular cloud. Clockwise from upper left: from $2 \mathrm{MASS}$ data, from $\tau_{353}$, from Schlegel et al. (1998), and from $\mathcal{R}$.
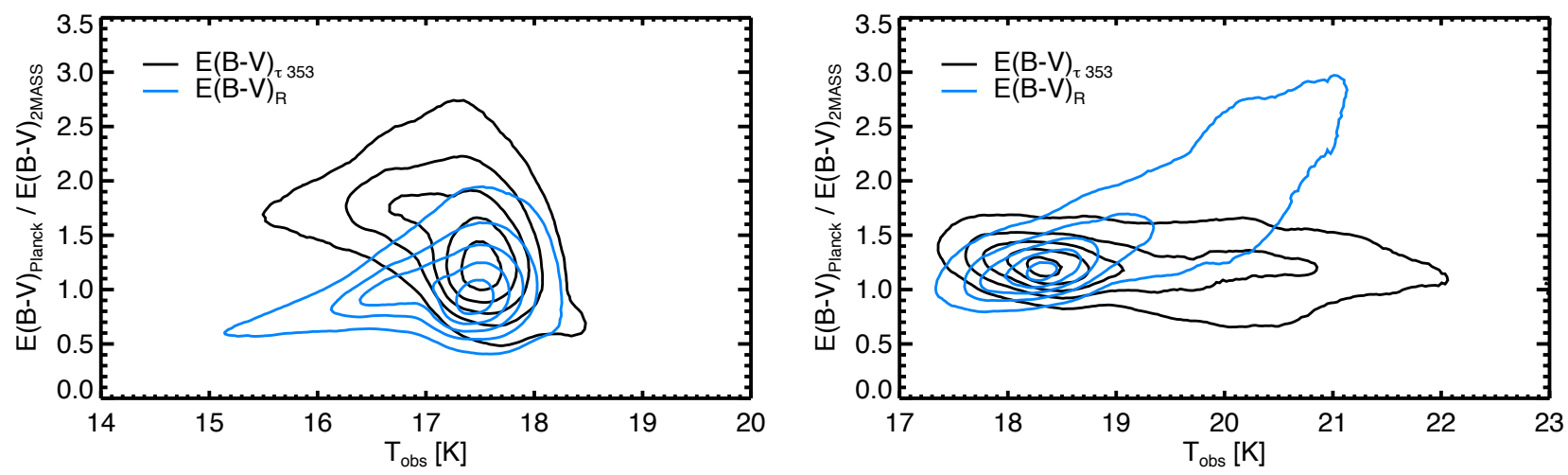

Fig. 25. Two-dimensional histogram of $E(B-V)_{\tau 353} / E(B-V)_{2 \mathrm{MASS}}$ (black contours) and $E(B-V)_{\mathcal{R}} / E(B-V)_{2 \mathrm{MASS}}$ (blue contours) as a function of $T_{\text {obs }}$, for the Taurus (left) and $\rho$ Ophiuchi (right) molecular clouds. The maps used are those shown in Figs. 23 and 24.

$E(B-V)_{\mathcal{R}} / E(B-V)_{2 \mathrm{MASS}}$ decreases. This greatly reduces the contrast across the map of $E(B-V)_{\mathcal{R}}$.

In $\rho$ Ophiuchi, there is a similar scale difference in the typical $E(B-V)_{\tau 353} / E(B-V)_{2 \mathrm{MASS}}$ and an upturn toward lower $T_{\mathrm{obs}}$. Unlike in Taurus, $L_{\mathrm{H}}$ is not generally depressed. There is a correlation of $E(B-V)_{\mathcal{R}} / E(B-V)_{2 \mathrm{MASS}}$ with $T_{\mathrm{obs}}$ as expected if $T_{\mathrm{obs}}$ reflects changes in the strength of radiation. Without an independent measure of changes in the ISRF, $\mathcal{R}$ is not a reliable quantitative tracer of the dust column density.

\subsection{Discussion}

As in the comparison of column density measures from dust emission with gas column density, the comparison with dust 
Table 5. Pearson correlation coefficient with the $E(B-V)$ map obtained with colour excess using 2MASS data.

\begin{tabular}{lccc}
\hline \hline Cloud & $E(B-V)_{\tau 353}$ & $E(B-V)_{\mathcal{R}}$ & $E(B-V)_{\mathrm{SFD}}$ \\
\hline Taurus & 0.86 & 0.75 & 0.67 \\
$\rho$ Ophiuchi & 0.95 & 0.70 & 0.79 \\
\hline
\end{tabular}

extinction leads us to the conclusion that $\mathcal{R}$ is a slightly better tracer of $E(B-V)$ for diffuse low column density lines of sight. This tracer, of particular interest for extragalactic studies, is the product that we call $E(B-V)_{\text {xgal }}$ in the Planck Legacy Archive (PLA; Appendix F). In addition to its usefulness in estimating Galactic $E(B-V)$ for extragalactic studies, it can also be used to study the structure of the diffuse ISM in regions where the CIBA is dominating the fluctuations at small scales. We stress that $E(B-V)_{\mathrm{xgal}}$ should not be used for $E(B-V)>0.3$, where attenuation effects on $\mathcal{R}$ become important. In particular, this counter-indication applies in molecular clouds and star forming regions where $\mathcal{R}$ traces not only the dust column density but also changes in the ISRF arising from attenuation and/or local sources of radiation. In line with the discussion in Sect. 5.5, in such regions $E(B-V)_{\text {xgal }}$ should not be used.

However, in such regions $\tau_{353}$ is well correlated with $E(B-V)_{2 \mathrm{MASS}}$, suggesting an alternative tracer. Again there is an issue with absolute amount, as in the conversion of $\tau_{353}$ to $N_{\mathrm{H}}$, relating to changes in $\sigma_{\mathrm{e} 353}$. Adopting the scaling factor estimated using the correlation with quasars for more diffuse lines of sight $\left(E(B-V) / \tau_{353}=1.49 \times 10^{4}-\right.$ see Fig. 22$)$ along with the $\tau_{353}$ maps could systematically overestimate $E(B-V)$, and this could be exacerbated in the most dense regions where $\sigma_{\mathrm{e} 353}$ increases further.

\section{Planck dust products and comparisons with forerunners}

\subsection{Description of products}

As described more fully in Appendix F, the following maps are available in the PLA: the three MBB parameters $T_{\mathrm{obs}}, \beta_{\mathrm{obs}}$, and $E(B-V)_{\mathrm{xgal}}$, together with the associated uncertainty maps. The map of dust integrated intensity, $\mathcal{R}$, can be obtained readily from the three MBB parameter maps using the analytical expression of Eq. (10).

$E(B-V)_{\mathrm{xgal}}$, a scaled version of $\mathcal{R}$, was obtained from MBB parameters of a fit to data from which point sources have been removed (see Sect. 3.4 and Appendix D). The map of this $\mathcal{R}$ can therefore be obtained by dividing the $E(B-V)_{\mathrm{xgal}}$ map by the conversion factor from Sect. 6.1 (Fig. 22, upper).

The main limitations on the Planck dust products from the data themselves are related to the IRAS data. There is some residual striping in the $3000 \mathrm{GHz}$ IRAS data that propagates mainly into the map of $T_{\text {obs }}$. There is also about $4 \%$ of the sky that was not observed by IRAS (Beichman et al. 1988). This area was filled with DIRBE data (Miville-Deschênes \& Lagache 2005). Finally, we stress that the dust model is based on data that unavoidably include the CIBA.

\subsection{Modelling dust emission: comparison of Planck with Finkbeiner et al. (1999)}

One of the important applications of these parameter maps will be to combine them using Eq. (6) to model the SED of the dust emission in the submillimetre range.
A benchmark for comparison is the parametric SED model developed by Finkbeiner et al. (1999). Motivated by data from the FIRAS experiment, they modelled the dust emission as the sum of two MBBs. Each MBBs component is in principle characterized by three parameters. However, by adopting the view that some parameters (or related ratios) are global and by using the constraints provided by thermal coupling to the same radiation field at a given sky position (like Eqs. (10) and (11)) so that the two temperatures are coupled, their model is simplified to only two degrees of freedom instead of six. Thus over the whole sky, fitting IRAS and lower resolution DIRBE data, the model can be summarized by two templates corresponding to the two degrees of freedom: the total dust optical depth at $100 \mu \mathrm{m}$ at a resolution of 6!. 1 and a dust temperature map (for either component) at a resolution of several degrees (it is almost constant at high Galactic latitude).

The dust model can be improved significantly by exploiting the Planck data. The exploration of the parametrization of the dust SED done previously at $7^{\circ}$ resolution with FIRAS data can be done at $5^{\prime}$ with much better sensitivity. With the recalibration of the Planck 545 and $857 \mathrm{GHz}$ data we have shown that a single MBB is a good representation of the dust SED over the $353-3000 \mathrm{GHz}$ frequency range, well within the relatively large calibration uncertainties of the data (about $10 \%$ at 545, 857, and $3000 \mathrm{GHz}$ ) (Sect. 3.3, Fig. 11). Planck Collaboration Int. XVII (2014) reached the same conclusion. Even though the Planck dust model assumes that the dust emission can be modelled by a single MBB from 353 to $3000 \mathrm{GHz}$, it has one extra degree of freedom compared to Finkbeiner et al. (1999) because $\tau_{353}, T_{\mathrm{obs}}$, and $\beta_{\text {obs }}$ are estimated at each sky position. Finally, the parameter maps are at higher resolution $\left(5^{\prime}, 5^{\prime}\right.$, and $30^{\prime}$, respectively) and are less noisy, providing a very tight description of the data over that frequency range. With frequency coverage spanning the SED, we can also measure the radiance $\mathcal{R}$.

Figure 26 shows the ratio of the predicted brightness at $353 \mathrm{GHz}$ from the Finkbeiner et al. (1999) dust model (model 7) and that from the Planck model, both at $30^{\prime}$ resolution.

Although the global ratio of the two maps is compatible with one, there are variations at all scales much larger than the uncertainties of the Planck model. Local variations larger than $30 \%$ are seen all over the sky, especially in the Galactic plane; the outer Galaxy is significantly underpredicted in the Finkbeiner et al. (1999) model while the inner Galaxy is too bright. Because the Planck dust model is a particularly tight representation of the Planck $353 \mathrm{GHz}$ data (Fig. 11), the same discrepancies are seen by comparing the Finkbeiner et al. (1999) model, integrated in the Planck bandpass, directly with the $353 \mathrm{GHz}$ Planck data.

The Planck dust model produces an accurate $353 \mathrm{GHz}$ map almost free of instrumental noise (but recall that the model includes the effects of the CIBA). That model map along with $T_{\text {obs }}$ can be the basis for extrapolation to lower frequencies, assuming that the appropriate $\beta_{\mathrm{obs}, \mathrm{mm}}$ can be identified.

\subsubsection{Frequency range of application}

We recall that our fit was done using data from 353 to $3000 \mathrm{GHz}$. Extrapolating the model outside this range is not recommended. At higher frequencies the dust emission is known to be in excess with respect to the big grain $\mathrm{MBB}$, due to the emission from smaller, stochastically heated, grains (see, e.g., Draine \& Li 2007; Compiègne et al. 2011).

At frequencies below $353 \mathrm{GHz}$ the dust SED seems to be flatter than that found for the frequency range here, i.e., from the tests that we have made, extrapolation of the dust 


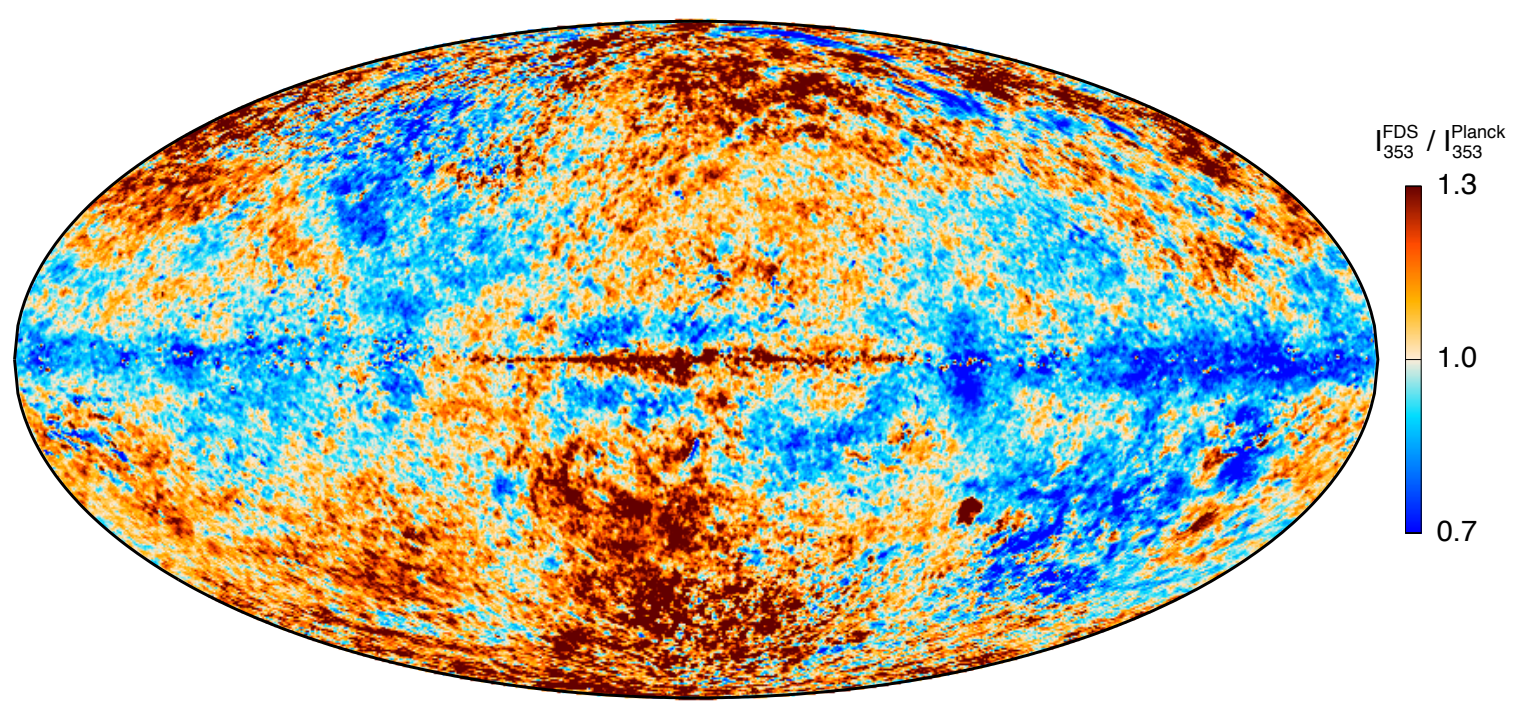

Fig. 26. Ratio of the dust specific intensity at $353 \mathrm{GHz}$ from the Finkbeiner et al. (1999) and Planck models, $I_{353}^{\mathrm{FDS}} / I_{353}^{\text {Planck }}$, using maps smoothed to $30^{\prime}$.

model underpredicts the unmodelled Planck dust emission at lower frequencies. This is in accord with the results of Planck Collaboration Int. XVII (2014) for the south Galactic pole area where $\beta_{\text {obs,mm }}=1.54 \pm 0.03$ between 100 and $353 \mathrm{GHz}$ and $\beta_{\text {obs,FIR }}=1.65 \pm 0.10$ at higher frequencies. The spectral index of dust between 100 and $353 \mathrm{GHz}$ estimated over the whole sky using the Commander-Ruler code (Planck Collaboration XII 2014) has a mean value of 1.49 , thus also significantly flatter, and interesting variations (their Fig. 16) that are similar though not identical to what we see in our map of $\beta_{\text {obs }}$ (Fig. 9, lower). While further discussion is beyond the scope of this paper, it is clear that extrapolating the current model to frequencies lower than $353 \mathrm{GHz}$ needs to be approached with caution.

\subsection{Extinction: comparison of Planck with Schlegel et al. (1998)}

One of the expected uses of the Planck dust products presented here is to estimate reddening for extragalactic studies. Here we evaluate how the Planck $E(B-V)_{\text {xgal }}$ map compares with the widely-used $E(B-V)_{\mathrm{SFD}}$ map from Schlegel et al. (1998).

The $E(B-V)$ map of Schlegel et al. (1998) is proportional to their map of $\tau_{3000}$, obtained assuming a constant $\beta_{\text {obs }}$ and with $T_{\text {obs }}$ estimated with low resolution, low sensitivity DIRBE data. The proportionality factor $E(B-V) / \tau_{3000}$ was estimated by correlating $\tau_{3000}$ with colour excess measurements on 389 galaxies, assuming $R_{V}=3.1$ and the extinction curve from Cardelli et al. (1989) and O'Donnell (1994). This factor $E(B-V) / \tau_{3000}$ has been checked by different probes of extinction and for much larger samples. These studies all used SDSS data and showed that, globally, for $E(B-V)<0.5$, the regime of interest for extragalactic studies, $E(B-V)_{\mathrm{SFD}}$ is precise to $15 \%$, though not a fully consistent picture. Using reddening of quasars, Mörtsell (2013) concluded that $E(B-V)_{\mathrm{SFD}}$ underestimates $E(B-V)$ by $20 \%$ at low $E(B-V)$ values. Using reddening measurements of elliptical galaxies Peek \& Graves (2010) found local variations of $E(B-V)_{\mathrm{SFD}} / E(B-V)_{\text {elliptical but no systematic bias }}$ of the $E(B-V)_{\mathrm{SFD}}$ map. Using reddening of stars, Schlafly et al. (2010) and Schlafly \& Finkbeiner (2011) concluded that $E(B-V)_{\mathrm{SFD}}$ overestimates $E(B-V)$ by $14 \%$ but with spatial variations of the normalization of the order of $10 \%$ which might be attributed to biases in the dust temperature map of Schlegel et al. (1998). Jones et al. (2011) used SDSS colours of M dwarfs to estimate the Galactic extinction properties. In their comparison to Schlegel et al. (1998) they often find $E(B-V)$ values lower than $E(B-V)_{\mathrm{SFD}}$ at lower Galactic latitudes but mention that this could be due to the fact that extinction toward stars does not trace the full line-of-sight dust column density, an effect that could be present in the analysis of Schlafly et al. (2010); Schlafly \& Finkbeiner (2011). At high Galactic latitudes Jones et al. (2011) report a number of lines of sight where $E(B-V)_{\mathrm{SFD}}$ seems to underestimate $E(B-V)$.

Based on near-infrared data in brighter areas $(E(B-V)>$ 1.5), Arce \& Goodman (1999) and Cambrésy et al. (2001) found that $E(B-V)_{\mathrm{SFD}}$ overestimates $E(B-V)$ systematically by more than $30 \%$. One possible interpretation is an increase of the dust emission efficiency, $\sigma_{\mathrm{a}}$, relative to the dust absorption cross section, $\overline{\sigma_{\mathrm{a}}}$, in the dense medium, potentially caused by changes in the grain structure, similarly to what is seen with $\tau_{353} / N_{\mathrm{H}}$ in Fig. 20, upper. All of these studies reveal variations of the ratio $E(B-V)_{\mathrm{SFD}} / E(B-V)_{\mathrm{SDSS}}$ that depend on position on the sky, on methodology (stars, galaxies, quasars), and on the range of $E(B-V)$. There seem to be systematic trends where $E(B-V)_{\mathrm{SFD}}$ overestimates reddening in dense regions and underestimates reddening in diffuse areas at high Galactic latitudes.

The Planck $E(B-V)_{\mathrm{xgal}}$ and $E(B-V)_{\mathrm{SFD}}$ are compared in Fig. 27, for the low $N_{\mathrm{HI}}$ mask (Fig. 3) corresponding to $N_{\mathrm{HI}}<$ $2 \times 10^{20} \mathrm{~cm}^{-2}$ and low IVC emission. The comparison is done after smoothing $E(B-V)_{\mathcal{R}}$ to 6 '. 1 , the resolution of $E(B-V)_{\mathrm{SFD}}$. The correlation between the two maps is excellent with $E(B-$ $V)_{\mathrm{SFD}}=0.92 E(B-V)_{\mathcal{R}}-0.003$. The dispersion around the correlation is $7 \%$. In relative terms $E(B-V)_{\mathrm{SFD}}$ underestimates $E(B-V)_{\mathrm{xgal}}$ by $8 \%$ but in absolute terms the underestimate is more as there is also a negative offset of $0.003 \mathrm{mag}$.

Our result depends on our choice of using $E(B-V)$ of quasars to calibrate $\mathcal{R}$. Given the variety of methodologies used in the previous studies that have examined the calibration of the high latitude extinction (Schlafly et al. 2010; Schlafly \& Finkbeiner 2011; Peek \& Graves 2010; Jones et al. 2011; Mörtsell 2013), each having their own biases, the agreement is excellent. 


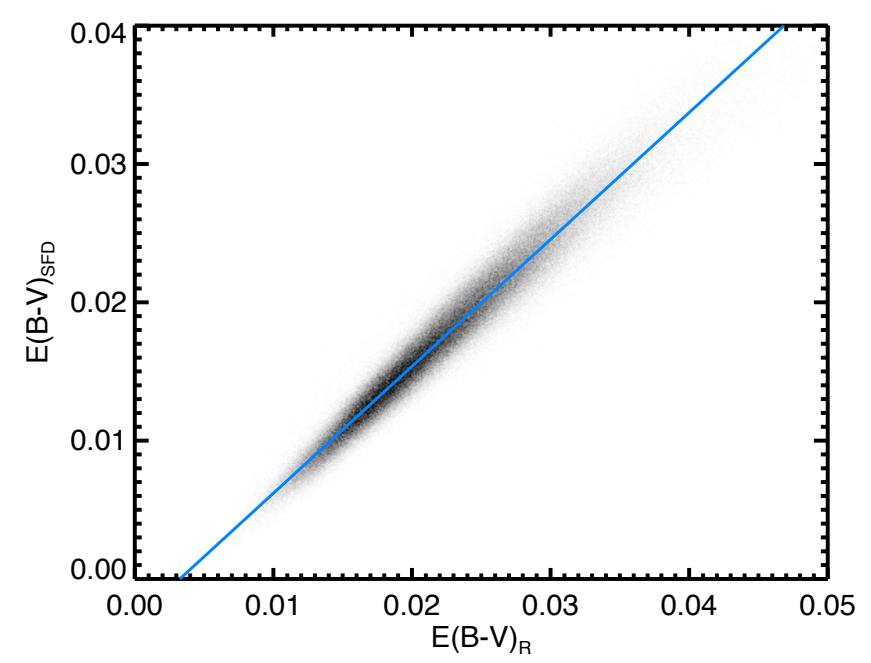

Fig. 27. Comparison of $E(B-V)_{\text {xgal }}$ from Planck (obtained from $\mathcal{R}$, smoothed to 6.1) and $E(B-V)_{\mathrm{SFD}}$ from Schlegel et al. (1998) for the low $N_{\mathrm{HI}}$ mask (Fig. 3). The greyscale gives the density of points and the solid line is the linear regression: $E(B-V)_{\mathrm{SFD}}=0.92 E(B-V)_{\mathrm{xgal}}-$ 0.003 .

\section{Conclusion}

We have presented an all-sky model of dust emission based on Planck and IRAS data at $5^{\prime}$ resolution, covering the frequency range from 353 to $3000 \mathrm{GHz}$. We fit the data at each pixel of the HEALPix $N_{\text {side }}=2048$ grid assuming a modified blackbody model. The parameters were estimated using a $\chi^{2}$ minimization, in two steps. First the data were smoothed to $30^{\prime}$ and $\tau_{353}, T_{\text {obs }}$, and $\beta_{\text {obs }}$ were estimated in a three-parameter fit. Then, using the spectral index $\beta_{\text {obs }}$ at $30^{\prime}$ as a fixed parameter, $\tau_{353}$ and $T_{\text {obs }}$ were estimated at $5^{\prime}$. We showed that this method minimizes the effect of noise on the determination of the parameters (especially on the $T_{\mathrm{obs}}-\beta_{\mathrm{obs}}$ degeneracy in faint parts of the sky).

Over the whole sky, the mean and standard deviation of $T_{\mathrm{obs}}$ and $\beta_{\text {obs }}$ are [19.7, 1.4] K and [1.62, 0.10], respectively. The uncertainties of each parameter are about 3-6\%. We showed using Monte Carlo simulations that the uncertainties are dominated by the CIBAs, which are highly correlated within the Planck bands but only at a $30 \%$ level between Planck and IRAS $3000 \mathrm{GHz}$. The CIBA is the dominant source of uncertainty after smoothing the data to $30^{\prime}$ due to its non-white power spectrum $\left(C_{\ell} \propto l^{-1}\right)$.

This Planck dust model reproduces the Planck data well within the noise level at all frequencies. Comparison of the Finkbeiner et al. (1999) dust model with the new data and model shows only broad agreement, with variations of the order of $30 \%$ at all scales.

We found an increase of the dust opacity, $\tau_{353} / N_{\mathrm{H}}$, by a factor of two from the diffuse to the higher column density (denser) ISM. Empirically, this increase is associated with a decrease in $T_{\text {obs }}$; because grains are in equilibrium with the interstellar radiation field, we interpret this as a response to the increased dust emissivity. We also noted an excess of dust emission and opacity at $\mathrm{H}$ I column densities lower than $10^{20} \mathrm{~cm}^{-2}$ that might be attributed to dust in the warm ionized medium (WIM).

The combination of Planck and IRAS data allowed us to model the dust emission from the Rayleigh-Jeans regime over the peak of the SED to the Wien side and therefore to estimate the dust integrated specific intensity or radiance $\mathcal{R}$ for each line of sight. We also presented a map of the specific dust luminosity $L_{\mathrm{H}}$ by normalizing $\mathcal{R}$ with respect to H I (Eq. (14)). Given thermal equilibrium emission, this is a direct tracer of $U$, the average strength of the interstellar radiation field (weighted by dust absorption opacity) on each line of sight. This $L_{\mathrm{H}}$ map reveals an increase of $U$ in the inner Galaxy, in active star forming regions, and in the Magellanic Clouds. The map of $L_{\mathrm{H}}$ was shown to be notably different from that of $T_{\mathrm{obs}}$, indicating that $T_{\mathrm{obs}}$ is not a simple tracer of $U$ as often assumed. This is especially true at high Galactic latitudes where it was found that $L_{\mathrm{H}}$ is fairly uniform and $T_{\mathrm{obs}}$ depends (inversely) on the opacity $\tau_{353} / N_{\mathrm{HI}}$, confirming early Planck results (Planck Collaboration XXIV 2011). This reveals that $\tau_{353}$ is not the most reliable estimator of column density in the diffuse ISM. The spatial variations of $T_{\text {obs }}$ observed in the high-latitude sky appear to be a response to variations of the dust emission opacity resulting in grains of different equilibrium temperature even when exposed to the same $U$. The analysis at high Galactic latitude is consistent with $U$ being fairly uniform, so that $\mathcal{R}$ is a good estimator of column density and can be used to estimate $E(B-V)$ there.

On the other hand, in molecular clouds we showed that variations of $T_{\mathrm{obs}}$ are dominated by the effect of attenuation of the interstellar radiation and/or local sources of heating photons. In this type of environment, where the amplitude of the CIBA is negligible, $\tau_{353}$ is a better estimator of column density than $\mathcal{R}$, but the scale depends on the adopted opacity. Compared to the lower resolution work of Schlegel et al. (1998), the MBB analysis of Planck data in this paper provides estimates of $T_{\mathrm{obs}}$ at $5^{\prime}$ resolution and thus an improved higher-resolution estimate of $\tau_{353}$, especially in high-contrast molecular regions where the dust temperature and column density vary markedly at small scales.

The Planck dust model was used to produce a map to measure Galactic dust reddening for extragalactic studies at high Galactic latitude, $E(B-V)_{\mathrm{xgal}}$. This map was based on the radiance $\mathcal{R}$ and calibrated by comparison with SDSS reddening measurements of quasars. The correlation of $E(B-V)_{\text {xgal }}$ with the $E(B-V)$ map of Schlegel et al. (1998) is very tight for $N_{\mathrm{H}}<2 \times 10^{20} \mathrm{~cm}^{-2}$, but has a slope significantly different than one, in the sense that the $E(B-V)$ map of Schlegel et al. (1998) underestimates $E(B-V)$ by $8 \%$ in the diffuse ISM. We stress that $E(B-V)_{\mathrm{xgal}}$ is reserved for extragalactic studies; it should not be used to estimate reddening in lines of sight where $E(B-V)>0.3$, i.e., where attenuation effects on $\mathcal{R}$ become important. There we recommend the map of $\tau_{353}$ multiplied by the $E(B-V) / \tau_{353}$ ratio also calibrated using quasars. However, systematic decreases of scale can arise from region to region, and even locally within a region, because of the increases in the opacity $\sigma_{\mathrm{e} 353}$ that, empirically, accompany increase in (column) density.

The Planck dust products $\left(\tau_{353}, T_{\mathrm{obs}}, \beta_{\mathrm{obs}}, \mathcal{R}\right.$ and $\left.E(B-V)_{\mathrm{xgal}}\right)$ are available on the PLA (see Appendix F).

Acknowledgements. The development of Planck has been supported by: ESA; CNES and CNRS/INSU-IN2P3-INP (France); ASI, CNR, and INAF (Italy); NASA and DoE (USA); STFC and UKSA (UK); CSIC, MICINN, JA and RES (Spain); Tekes, AoF and CSC (Finland); DLR and MPG (Germany); CSA (Canada); DTU Space (Denmark); SER/SSO (Switzerland); RCN (Norway); SFI (Ireland); FCT/MCTES (Portugal); and PRACE (EU). A description of the Planck Collaboration and a list of its members, including the technical or scientific activities in which they have been involved, can be found at http://www.sciops.esa.int/index.php? project $=$ planck\&page $=\mathrm{Pl}$ lanck_Collaboration. The research leading to these results has received funding from the European Research Council under the European Union's Seventh Framework Programme (FP7/2007-2013)/ERC grant agreement No. 267934. Funding for the SDSS and SDSS-II has been provided by the Alfred P. Sloan Foundation, the Participating Institutions, the National Science Foundation, the US Department of Energy, the National Aeronautics and Space Administration, the Japanese Monbuk agakusho, the Max Planck Society, and the Higher Education Funding Council for England. The SDSS web 

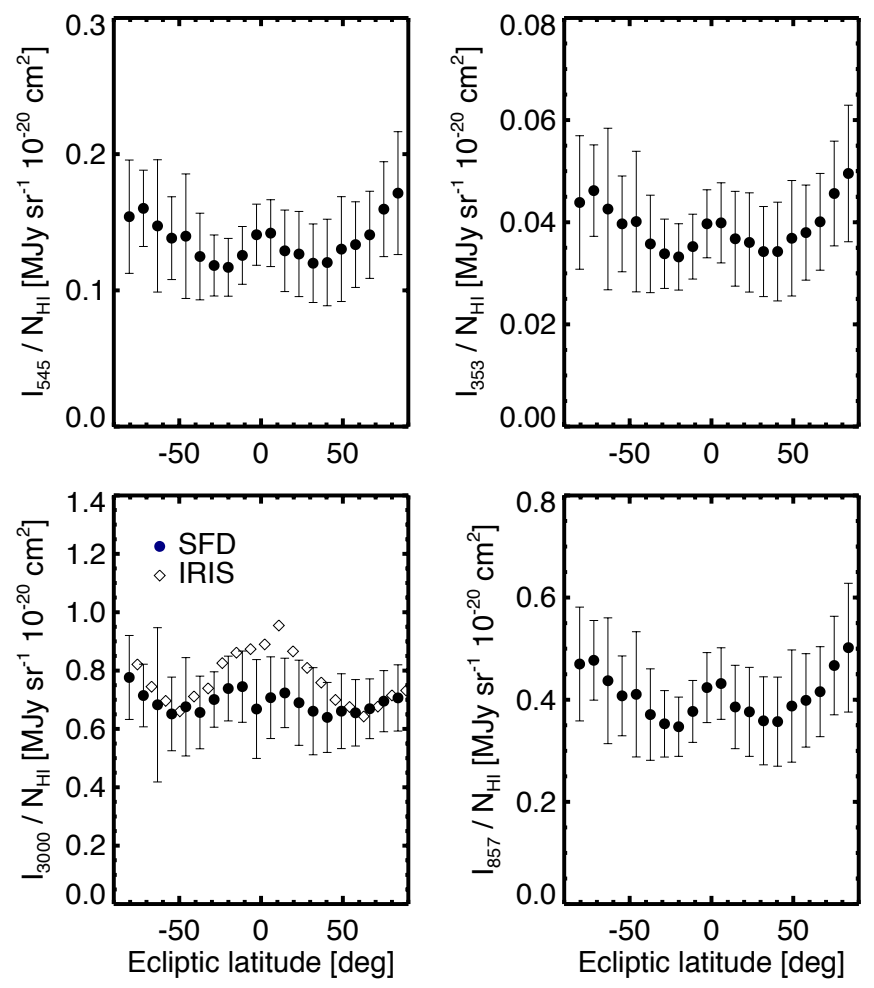

Fig. A.1. Dust emissivity $I_{v} / N_{\mathrm{HI}}$ as a function of ecliptic latitude for $3000 \mathrm{GHz}$ (lower left), $857 \mathrm{GHz}$ (lower right), $545 \mathrm{GHz}$ (upper left) and $353 \mathrm{GHz}$ (upper right). Each point gives the average and standard deviation (error bar) of all pixels in the corresponding bin in ecliptic latitude (IRIS error bars omitted for clarity). These plots were obtained using data smoothed to $1^{\circ}$ and selecting pixels with $N_{\mathrm{HI}}<3 \times 10^{20} \mathrm{~cm}^{-2}$.

site is http://www.sdss.org/. The SDSS is managed by the Astrophysical Research Consortium for the Participating Institutions. The Participating Institutions are the American Museum of Natural History, Astrophysical Institute Potsdam, University of Basel, University of Cambridge, Case Western Reserve University, University of Chicago, Drexel University, Fermilab, the Institute for Advanced Study, the Japan Participation Group, Johns Hopkins University, the Joint Institute for Nuclear Astrophysics, the Kavli Institute for Particle Astrophysics and Cosmology, the Korean Scientist Group, the Chinese Academy of Sciences (LAMOST), Los Alamos National Laboratory, the Max-PlanckInstitute for Astronomy (MPIA), the Max-Planck-Institute for Astrophysics (MPA), New Mexico State University, Ohio State University, University of Pittsburgh, University of Portsmouth, Princeton University, the United States Naval Observatory, and the University of Washington. Some of the results in this paper have been derived using the HEALPix package.

\section{Appendix A: Zodiacal emission}

\section{A.1. Zodiacal emission correction at $100 \mu \mathrm{m}$}

Zodiacal emission (ZE) is a component that is difficult to remove from the data as it is changing with sky position as well as time of observation. A detailed model has been built to correct the Planck maps for ZE (Planck Collaboration XIV 2014). For the IRAS $100 \mu \mathrm{m}$ map, as mentioned in Sect. 2.2, the IRIS and SFD maps were not corrected for ZE in the same way. The impact of the different $\mathrm{ZE}$ correction for these two maps can be appreciated from Fig. A. 1 where the dust emissivity $I_{v} / N_{\mathrm{H}}$ is plotted for the four frequencies, using data smoothed to $1^{\circ}$ and selecting only pixels with $N_{\mathrm{HI}}<3 \times 10^{20} \mathrm{~cm}^{-2}$. In such a low column density regime, the correlation between dust emission and H I column density was shown to be tight (Fig. 5). Therefore, any systematic variation of $I_{v} / N_{\mathrm{HI}}$, especially at low angular resolution, can reveal emission from components other than $\mathrm{HI}$, for example the warm ionized medium (WIM) or residual ZE. To assess the latter, in Fig. A.1 the emissivity is plotted as a function of ecliptic latitude. For the Planck frequencies the emissivity is almost constant with ecliptic latitude, validating the Planck ZE removal. The SFD data also show a constant emissivity, the exception being the IRIS map that shows a systematic increase toward the ecliptic plane, indicative of residual ZE. This is why we implemented the procedure described in Sect. 2.2.

\section{A.2. Impact of zodiacal emission correction on dust parameters}

To evaluate the impact of the ZE on our analysis, we also compared our results on fitting parameters with those of Planck Collaboration Int. XVII (2014) for the same masked region that they studied, an area of $7500 \mathrm{deg}^{2}$ toward the south Galactic cap. This check is useful because there is a fundamental difference between the two analyses. Here the dust SED is modelled using the observed specific intensity for each pixel independently; results are therefore sensitive to uncertainties in the zero levels of the maps. On the other hand, Planck Collaboration Int. XVII (2014) correlated the dust maps with H I within regions $15^{\circ}$ in diameter; they showed that their results are insensitive to the zero level of the maps and to the $\mathrm{ZE}$ that is very uniform on $15^{\circ}$ scales.

To further evaluate the impact of the ZE correction on our analysis, we have explored different data configurations and compared our results and those of Planck Collaboration Int. XVII (2014) in the south Galactic pole area.

Given the lower-resolution results of Planck Collaboration Int. XVII (2014), we used data smoothed to $60^{\prime}$ on an $N_{\text {side }}=$ 128 grid. The comparison was done using different combinations of maps: IRIS or SFD at $100 \mu \mathrm{m}$ (at such low resolution, SFD is equivalent to the combined IRIS+SFD map, see Sect. 2.2) and maps with and without ZE removed for Planck. To be compatible with Planck Collaboration Int. XVII (2014), the fit was done using a fixed $\beta_{\mathrm{obs}}=1.65$. The results we obtained on $T_{\mathrm{obs}}$ and $\tau_{353} / N_{\mathrm{HI}}$ for this south Galactic cap region are compiled in Table A. 1 . The differences in $T_{\mathrm{obs}}$ and $\tau_{353} / N_{\mathrm{H} \text { I }}$ between data configurations are limited, within the standard deviation observed over the region. They are especially small for $T_{\text {obs }}$ that shows variations of less than $5 \%$ between data configurations. The largest effect is from the removal of the $\mathrm{ZE}$ in the Planck data that reduces $\tau_{353}$ by $15 \%$. The impact of the choice of IRIS or SFD on $\tau_{353}$ is more limited; fitting the data with SFD produces a $\tau_{353}$ about $3 \%$ higher than with IRIS. Nevertheless and even though there is a general good spatial correlation between the maps of $T_{\text {obs }}$ and $\tau_{353} / N_{\mathrm{HI}}$ obtained with the two methods, care should be taken in comparing them in greater detail. Contrary to the results obtained using a fit of the observed specific intensity, the results of Planck Collaboration Int. XVII (2014) are not sensitive to dust emission associated with the WIM that is not spatially correlated with H I and to the CIBAs. In addition, Planck Collaboration Int. XVII (2014) showed that in the southern Galactic cap area there are H I clouds at local velocities that do not have associated dust emission. These effects produce spatial variations of $T_{\mathrm{obs}}$ and $\tau_{353} / N_{\mathrm{HI}}$ computed with the two methods. Even with these caveats, there is a good agreement between the two analyses. In particular we note that the data configuration combining Planck (ZE removed) together with the SFD map at large scales (the equivalent of the $100 \mu \mathrm{m}$ map built in Eq. (1)) has $\left\langle\tau_{353} / N_{\mathrm{HI}}\right\rangle$, the closest to the values found by Planck Collaboration Int. XVII (2014). 
Planck collaboration: Planck 2013 results. XI.

Table A.1. Data configuration exploration and comparison with result from Planck Collaboration Int. XVII (2014).

\begin{tabular}{lcccc}
\hline \hline & $\left\langle T_{\text {obs }}\right\rangle$ & $\sigma\left(T_{\text {obs }}\right)$ & $\left\langle\tau_{353} / N_{\mathrm{HI}}\right\rangle$ & $\sigma\left(\tau_{353} / N_{\mathrm{HI}}\right)$ \\
{$[\mathrm{K}]$} & {$[\mathrm{K}]$} & {$\left[\mathrm{cm}^{2} \mathrm{H}^{-1}\right]$} & {$\left[\mathrm{cm}^{2} \mathrm{H}^{-1}\right]$} \\
\hline Planck ZE rm, IRIS & 20.6 & 1.0 & $6.8 \times 10^{-27}$ & $1.8 \times 10^{-27}$ \\
Planck ZE rm, SFD & 20.2 & 0.8 & $7.0 \times 10^{-27}$ & $1.7 \times 10^{-27}$ \\
Planck, IRIS & 20.0 & 0.7 & $8.0 \times 10^{-27}$ & $1.6 \times 10^{-27}$ \\
Planck, SFD & 19.6 & 0.5 & $8.3 \times 10^{-27}$ & $1.6 \times 10^{-27}$ \\
Planck Collaboration Int. XVII (2014) & 19.7 & 0.9 & $7.3 \times 10^{-27}$ & $2.2 \times 10^{-27}$ \\
\hline
\end{tabular}

Notes. The average and standard deviation of $T_{\mathrm{obs}}$ and $\tau_{353} / N_{\mathrm{HI}}$ were computed on the south Galactic pole area studied in Planck Collaboration Int. XVII (2014). Data smoothed to $60^{\prime}\left(N_{\text {side }}=128\right)$ were used to compute the dust parameters. In this comparison a fixed $\beta_{\text {obs }}=1.65$ was assumed in the fit.

\section{Appendix B: The $\chi^{2}$ fit}

Adopting a reference frequency $v_{0}=353 \mathrm{GHz}$, the parameters $\tau_{v_{0}}, T_{\mathrm{obs}}$ and $\beta_{\mathrm{obs}}$ (see Eq. (6)) were found at each sky position $p$ by fitting the Planck and IRAS data $I_{v}(p)$. The MPFIT $\chi^{2}$ minimization routine was used (Markwardt 2009). Colour corrections due to finite bandpasses (Planck and IRAS) were taken into account explicitly at each iteration. To speed up the convergence of the fit, initial estimates of the parameters were provided: $\beta_{\text {obs }}$ was initialized to $1.65, T_{\text {obs }}$ was initialized to the colour temperature $T_{3000-857}$ obtained from the ratio of $I_{3000} / I_{857}$ and assuming $\beta_{\mathrm{obs}}=1.65$, and $\tau_{353}$ was initialized to $I_{3000} /\left[B_{3000}\left(T_{3000-857}\right) \times(3000 / 353)^{1.65}\right]$ for frequencies in $\mathrm{GHz}$.

The fit takes into account the calibration uncertainty of the data $\left(c_{v} I_{v}(p)\right.$ - see Table 1$)$, the uncertainty of the CMB removal estimated to be $c_{v} I_{\mathrm{CMB}}(p)$ where $I_{\mathrm{CMB}}$ is the SMICA map (Planck Collaboration XII 2014), the uncertainty of the offset $\left(\delta O_{v}-\right.$ see Table 1$)$, and the instrumental noise $n_{v}(p)$. For both IRAS and Planck, the instrumental noise is modulated inversely by the square root of the coverage map (number of times a sky pixel $p$ has been observed). All sources of uncertainty are added in quadrature:

$$
N_{v}(p)=\sqrt{c_{v}^{2} I_{v}(p)^{2}+c_{v}^{2} I_{\mathrm{CMB}}(p)^{2}+\left(\delta O_{v}\right)^{2}+n_{v}(p)^{2}} .
$$

\section{Appendix C: Impact of the CIBA on the dust parameters}

This section describes the Monte Carlo simulations done to quantify the impact of instrumental noise and the CIBA on the parameters of the MBB fit. Specifically, we simulated a single SED as the sum of dust emission, the CIBA, and instrumental noise, for data smoothed to different angular resolution.

The dust emission was modelled using typical values of dust parameters and taking into account the Planck and IRAS bandpasses. The noise level used at each frequency is the median value found in the low $N_{\mathrm{HI}}$ mask (Fig. 3), properly scaled as a function of the angular resolution considered. The CIBA levels in the Planck channels follow the parametrization of Planck Collaboration Int. XVII (2014) $\left(T_{\mathrm{CIB}}=18.3 \mathrm{~K}\right.$ and $\left.\beta_{\mathrm{CIB}}=1.0\right)$ normalized at $857 \mathrm{GHz}$ to the value given by Planck Collaboration XVIII (2011). The CIBA level at $3000 \mathrm{GHz}$ is that of Pénin et al. (2012). The scaling of the CIBA level with resolution was done assuming a power spectrum $C_{\ell} \propto \ell^{-1}$. The noise and CIBA levels for each frequency and each resolution are given in Table C.1.

Because galaxies at different redshifts have their peak emission at different frequencies, the structure of the CIB on the sky is only partly correlated between frequencies. Looking at
Planck Collaboration XVIII (2011) and Planck Collaboration XXIV (2011), who showed the residual brightness fluctuations in selected faint patches of the sky after removal of the interstellar dust emission traced by $21 \mathrm{~cm}$ emission, one can appreciate visually the level of spatial correlation of the CIBA between frequencies. The fluctuations are strongly correlated in the $857-353 \mathrm{GHz}$ range, slightly less at $3000 \mathrm{GHz}$. The level of frequency (de)coherence of the CIBA was quantified by Planck Collaboration XXX (2014) for $150<\ell<1000$. In the high-frequency channels of Planck they report a strong correlation: 0.95 between 857 and $545 \mathrm{GHz}, 0.91$ between 857 and $353 \mathrm{GHz}$, and 0.98 between 545 and $353 \mathrm{GHz}$. It is weaker between $3000 \mathrm{GHz}$ and the Planck frequencies: 0.36, 0.31, and 0.29 at 857,545 and $353 \mathrm{GHz}$ respectively. Planck Collaboration Int. XVII (2014) modelled the part of the CIBA correlated with the ones at $857 \mathrm{GHz}$ based on the results of Planck Collaboration XXX (2014). They found that it can be well fitted by a MBB function with $T_{\mathrm{CIB}}=18.3 \mathrm{~K}$ and $\beta_{\mathrm{CIB}}=1.0$. This parametrization is compatible with the one found in a similar study by Planck Collaboration XXIV (2011).

To model the CIBA, $c_{v}$, taking into account the interfrequency correlation, we have proceeded the following way. We made the assumption that $c_{\nu}$ is fully correlated in the Planck frequencies. Therefore, the CIBA in the Planck bands is simply modelled as a single random draw scaled by the CIBA levels:

$c_{v}=A^{\text {cor }} \sigma_{\mathrm{CIBA} v}$,

where $A^{\text {cor }}$ is drawn from a normal distribution with unit standard deviation and zero mean, and $\sigma_{\mathrm{CIBA} v}$ is the CIBA level at each Planck frequency given in Table C.1.

At $3000 \mathrm{GHz}$ the CIBA is not strongly correlated with the CIBA at Planck frequencies so it was divided into one part correlated with $857 \mathrm{GHz}$ and another part uncorrelated:

$c_{3000}=A^{\text {cor }} \sigma_{\text {CIBA } 3000}^{\text {cor }}+A^{\text {uncor }} \sigma_{\text {CIBA } 3000}^{\text {uncor }}$,

where $A^{\text {uncor }}$ represent a second random draw. The correlated part, $\sigma_{\mathrm{CIBA}}^{\text {cor }}$, is estimated using the $\left[T_{\mathrm{CIBA}}=18.3 \mathrm{~K}, \beta_{\mathrm{CIBA}}=\right.$ 1.0] parametrization, normalized with $c_{857}$. The uncorrelated part, $\sigma_{\text {CIBA }}^{\text {uncor }}$, is simply the quadratic complement to $\sigma_{\text {CIBA } 3000}$. At $5^{\prime}$ these contributions are $\sigma_{\text {CIBA } 3000}^{\text {cor }}=0.049 \mathrm{MJy} \mathrm{sr}^{-1}$ and $\sigma_{\text {CIBA } 3000}^{\text {uncor }}=0.087 \mathrm{MJy} \mathrm{sr}^{-1}$ highlighting the fact that the uncorrelated part is the dominant source of CIB fluctuations at $3000 \mathrm{GHz}$.

Noise was assumed to be uncorrelated in frequency, with levels given in Table C.1.

Depending on the situation, this procedure was executed with different dust parameters $\left(\tau_{353}, T_{\text {obs }}\right.$, and $\left.\beta_{\text {obs }}\right)$ and at different data resolutions. The fit of the simulated SED was done using the method that was used with the real data, including colour 
Table C.1. Noise and CIBA levels used for the Monte Carlo simulations.

\begin{tabular}{|c|c|c|c|c|c|c|c|c|}
\hline \multirow[b]{2}{*}{$\begin{array}{c}\theta \\
{[\operatorname{arcmin}]}\end{array}$} & \multicolumn{2}{|c|}{$353 \mathrm{GHz}$} & \multicolumn{2}{|c|}{$545 \mathrm{GHz}$} & \multicolumn{2}{|c|}{$857 \mathrm{GHz}$} & \multicolumn{2}{|c|}{$3000 \mathrm{GHz}$} \\
\hline & $\begin{array}{c}n \\
{\left[\mathrm{MJy} \mathrm{sr}^{-1}\right]}\end{array}$ & $\begin{array}{c}\sigma_{\mathrm{CIBA}} \\
{\left[\mathrm{MJy} \mathrm{sr}^{-1}\right]}\end{array}$ & $\begin{array}{c}n \\
{\left[\mathrm{MJy} \mathrm{sr}^{-1}\right]}\end{array}$ & $\begin{array}{c}\sigma_{\mathrm{CIBA}} \\
{\left[\mathrm{MJy} \mathrm{sr}^{-1}\right]}\end{array}$ & $\begin{array}{c}n \\
{\left[\mathrm{MJy} \mathrm{sr}^{-1}\right]}\end{array}$ & $\begin{array}{c}\sigma_{\mathrm{CIBA}} \\
{\left[\mathrm{MJy} \mathrm{sr}^{-1}\right]}\end{array}$ & $\begin{array}{c}n \\
{\left[\mathrm{MJy} \mathrm{sr}^{-1}\right]}\end{array}$ & $\begin{array}{c}\sigma_{\mathrm{CIBA}} \\
{\left[\mathrm{MJy} \mathrm{sr}^{-1}\right]}\end{array}$ \\
\hline 5 & $3.19 \times 10^{-2}$ & $1.60 \times 10^{-2}$ & $3.44 \times 10^{-2}$ & $4.36 \times 10^{-2}$ & $3.41 \times 10^{-2}$ & $1.00 \times 10^{-1}$ & $6.20 \times 10^{-2}$ & $1.00 \times 10^{-1}$ \\
\hline 15 & $3.63 \times 10^{-3}$ & $9.23 \times 10^{-3}$ & $3.91 \times 10^{-3}$ & $2.52 \times 10^{-2}$ & $3.86 \times 10^{-3}$ & $5.77 \times 10^{-2}$ & $6.93 \times 10^{-3}$ & $5.77 \times 10^{-2}$ \\
\hline 30 & $1.74 \times 10^{-3}$ & $6.52 \times 10^{-3}$ & $1.88 \times 10^{-3}$ & $1.78 \times 10^{-2}$ & $1.86 \times 10^{-3}$ & $4.08 \times 10^{-2}$ & $3.36 \times 10^{-3}$ & $4.08 \times 10^{-2}$ \\
\hline 60 & $8.61 \times 10^{-4}$ & $4.61 \times 10^{-3}$ & $9.29 \times 10^{-4}$ & $1.26 \times 10^{-2}$ & $9.20 \times 10^{-4}$ & $2.89 \times 10^{-2}$ & $1.67 \times 10^{-3}$ & $2.89 \times 10^{-2}$ \\
\hline 120 & $4.29 \times 10^{-4}$ & $3.26 \times 10^{-3}$ & $4.63 \times 10^{-4}$ & $8.89 \times 10^{-3}$ & $4.59 \times 10^{-4}$ & $2.04 \times 10^{-2}$ & $8.32 \times 10^{-4}$ & $2.04 \times 10^{-2}$ \\
\hline
\end{tabular}

Notes. The levels of noise, $n$, and the CIBA, $\sigma_{\mathrm{CIBA}}$, are given for each channel and for data smoothed to different angular resolution, $\theta$. The noise level is the median value of the noise in the low $N_{\mathrm{HI}}$ mask (see Fig. 3). The CIBA standard deviations at Planck frequencies are derived from the parametrization of Planck Collaboration Int. XVII (2014) $\left(T_{\mathrm{CIBA}}=18.3 \mathrm{~K}\right.$ and $\left.\beta_{\mathrm{CIBA}}=1.0\right)$ normalized at $857 \mathrm{GHz}$ with the value given by Planck Collaboration XVIII (2011). The value at $3000 \mathrm{GHz}$ is from Pénin et al. (2012). The scaling of $\sigma_{\mathrm{CIBA}}$ from the original measurements, made at 5.4 by Planck Collaboration XVIII (2011) and 4!3 by Pénin et al. (2012), to $5^{\prime}$ and $30^{\prime}$ was done assuming a CIBA power spectrum of $C_{\ell} \propto \ell^{-1}$.

corrections. Simulations were done with and without noise to quantify the specific effect of noise and the CIBA on the recovered parameters.

\section{Appendix D: Production of maps with point sources removed}

The $E(B-V)_{\text {xgal }}$ product, to be used for estimating Galactic reddening for extragalactic studies, is obtained with a fit of the dust model on a version of the Planck and IRAS data from which point sources have been removed. This section describes how the removal was done. Most of the software used in this process is part of the Planck Sky Model software package (PSM). A description of the PSM and how to download it can be found in Delabrouille et al. (2013).

\section{$D .1$. Making the mask containing the radio and $I R$ sources}

A source mask is made as a HEALPix map from the union of discs centred on a selected set of known FIR and radio sources. The FIR sources are compiled from the IRAS Point Source Catalog (PSC, Beichman et al. 1988) and the Faint Source Catalog (FSC, Moshir et al. 1992). Radio sources are derived from the WMAP catalog of point sources detected in the 9-year sky maps (Bennett et al. 2013), supplemented with a selection of extragalactic point sources detected by Planck at $100 \mathrm{GHz}$ (ERCSC, Planck Collaboration VII 2011) that are not in the WMAP catalog. Pixels within a $15^{\prime}$ radius of each source are masked.

\section{D.2. Grouping adjacent pixels for each source}

While most sources are isolated, in rare cases masks around two or more sources overlap. This results in larger, non-circular holes in the mask. As a first step, we divide the mask into a set of small connected holes (most but not all of which correspond to the masking of one single source). This is performed by the PSM routine GROUP_ADJACENT_PIXELS.

\section{D.3. Interpolating across the source}

Finally, we estimate the diffuse brightness in each of the small connected holes containing point sources using the PSM routine FILL_SMALLGAP. The interpolation is done using a minimum curvature surface (IDL routine MIN_CURV_SURF). This step produces the final, de-sourced maps.

\section{Appendix $E$ : Estimating $E(B-V)$ from colour excess measurements}

This section describes how $E(B-V)$ was estimated from the multi-colour SDSS measurements of quasars. The colour excess due to dust extinction is defined as

$$
\begin{aligned}
E(X-Y) & =A_{X}-A_{Y} \\
& =\left(m_{X}-m_{X 0}\right)-\left(m_{Y}-m_{Y 0}\right) \\
& =-2.5 \log \left(\frac{F_{X}}{F_{X 0}} \frac{F_{Y 0}}{F_{Y}}\right),
\end{aligned}
$$

where, for a given band $i, A_{i}$ is the extinction, $m_{i}$ the observed magnitude, $m_{i 0}$ the absolute magnitude, $F_{i}$ the observed flux, and $F_{i 0}$ the intrinsic flux of the source.

The quantity $F_{i 0}$ corresponds to the source flux density $f_{\lambda}$ convolved with the filter transmission $T_{i}(\lambda)$

$F_{i 0}=\int_{i} T_{i}(\lambda) f_{\lambda} \mathrm{d} \lambda$

while $F_{i}$ is the same quantity but affected by extinction;

$F_{i}=\int_{i} T_{i}(\lambda) f_{\lambda} 10^{-0.4 E(B-V) C_{\lambda}} \mathrm{d} \lambda$,

where $C_{\lambda}$ is the extinction curve for a given value of $R_{V}$ and normalized to $E(B-V)$ :

$C_{\lambda}=A_{\lambda} / E(B-V)$.

In what follows, we use $C_{\lambda}$ of Fitzpatrick (1999) and assume $R_{v}=3.1$.

Equations (E.1) to (E.5) relate the observed colour excess of a source in bands $X$ and $Y$ to its intrinsic spectrum $f_{\lambda}$, to the transmission of the filters $T_{i}$ and to the extinction of dust along the line of sight. As mentioned by Fitzpatrick \& Massa (2005), if the intrinsic spectrum of the source and the filter transmissions are known, the normalization of the extinction curve (i.e., $E(B-$ $V)$ ) can be estimated directly from the observed colour excess $E(X-Y)$.

An often overlooked fundamental fact follows from this description. Because the observed magnitudes $m_{i}$ are obtained with broad band filters, the measured value of $E(X-Y)$ for $X=B$ and $Y=V$ will in general be different from the normalization of the extinction curve $E(B-V)$ in Eq. (E.5). The observed colour excess is indeed a convolution of the source spectrum, the filter transmission and the extinction curve, and so there are bandpass 
Planck collaboration: Planck 2013 results. XI.
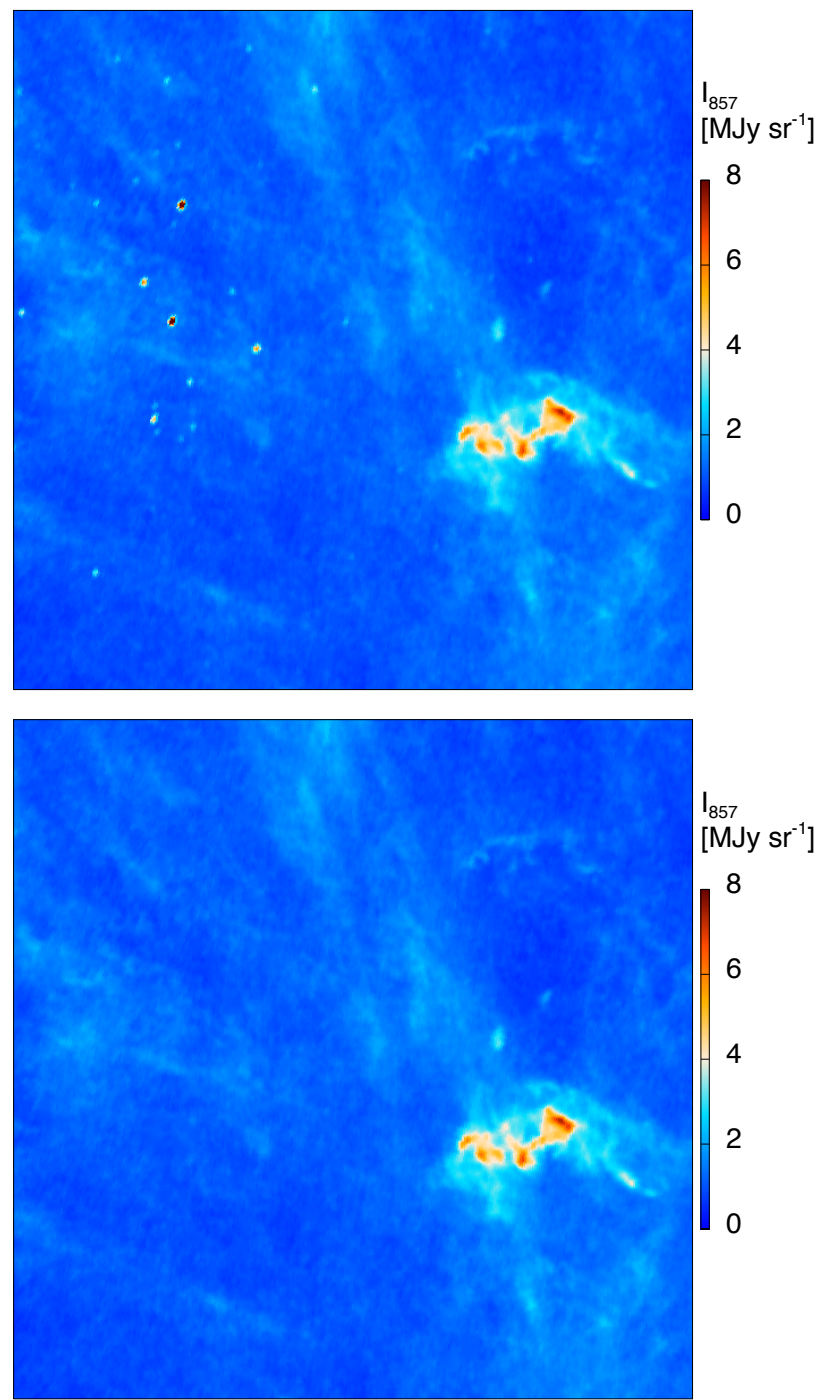
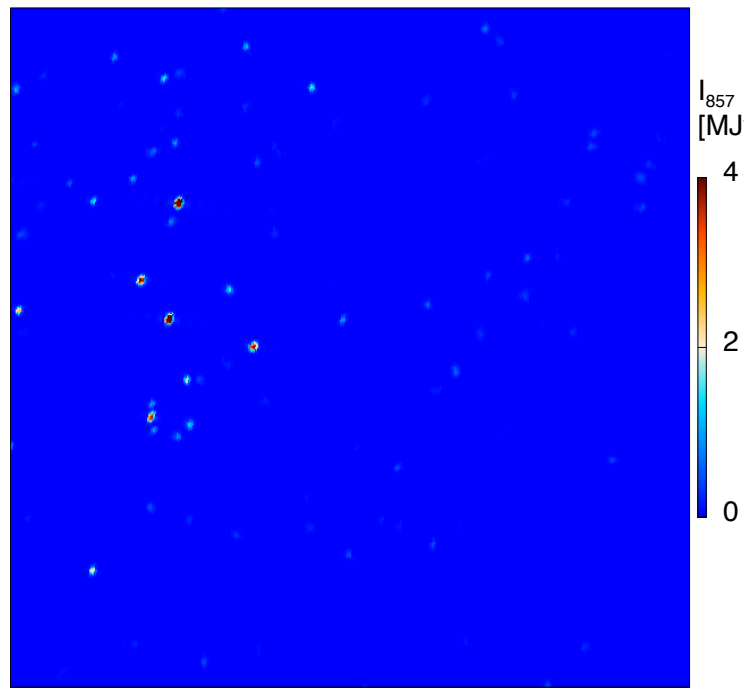

[MJy sr ${ }^{-1}$ ]

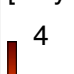

2

Fig. D.1. Example of the point source removal in a $10^{\circ} \times 10^{\circ}$ region centred on $l=260^{\circ}, b=75^{\circ}$; original map at $857 \mathrm{GHz}$ (upper), point source removed map (middle), and difference (lower).

corrections. We stress that the quantity $E(B-V)$ we are estimating here is independent of the spectrum of the background source. It is the value that is used to scale the extinction curve.
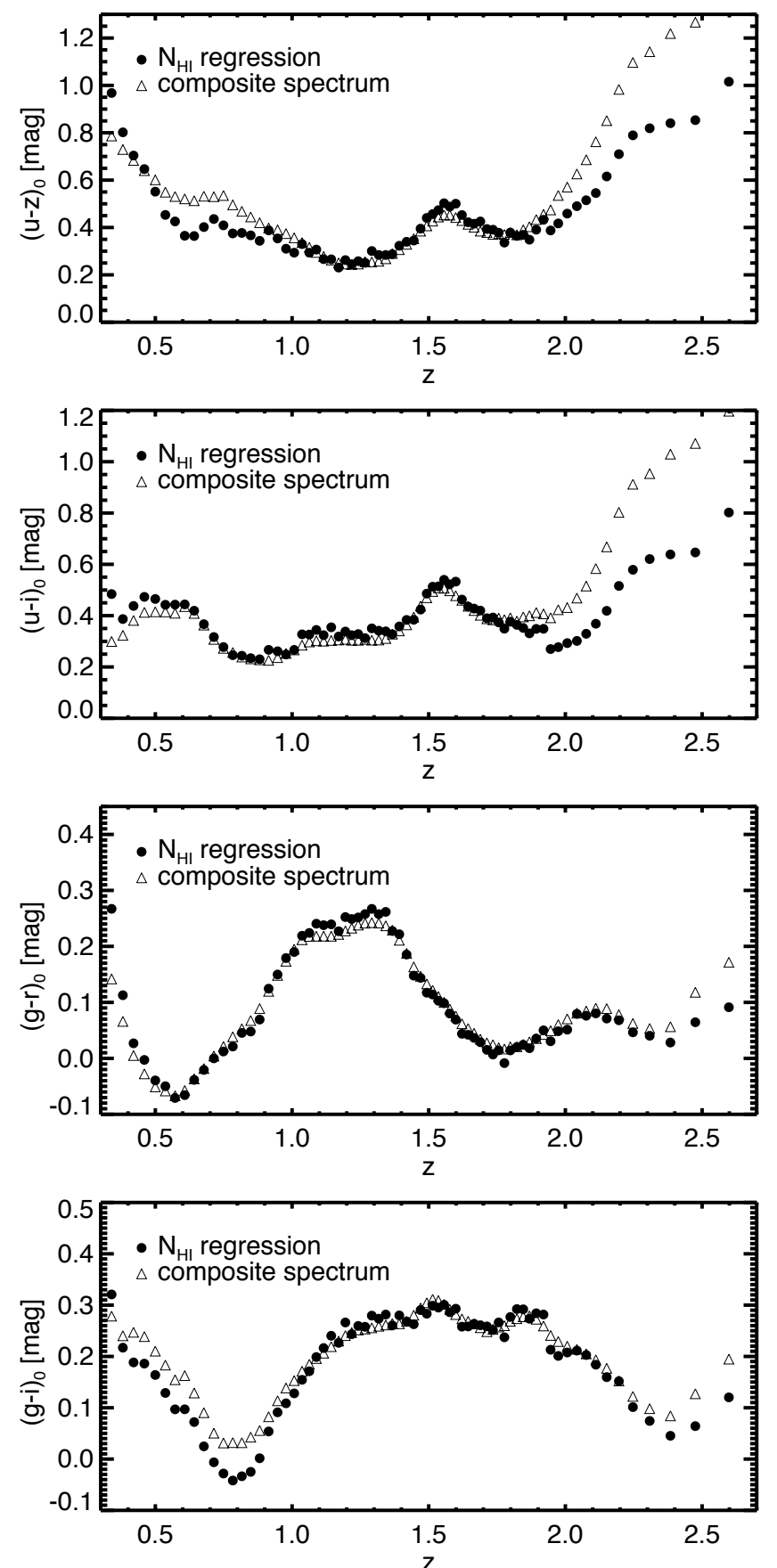

Fig. E.1. Intrinsic colour of quasars as a function of redshift for $(u-z)$, $(u-i),(g-r)$ and $(g-i)$, top to bottom. The black points give the intercept of the $\left(m_{X}-m_{Y}\right)$ vs. $N_{\mathrm{H}}$ relation in each bin of redshift (see Eq. (E.7)). The triangles give the intrinsic colour of quasars computed with the composite spectrum (see Eq. (E.8)).

From the SDSS quasar catalogue one can deduce directly the observed colours $\left(m_{X}-m_{Y}\right)$ but to estimate $E(B-V)$ using the formalism described in the previous section one also needs the source spectrum $f_{\lambda}$ and the intrinsic colour $\left(m_{X 0}-m_{Y 0}\right)$. One of the advantages of using quasars is that they are known to have a fairly constant spectrum. Below we use the composite quasar spectrum of Vanden Berk et al. (2001) for $f_{\lambda}$. The intrinsic colour $\left(m_{X 0}-m_{Y 0}\right)$ is estimated by correlating $\left(m_{X}-m_{Y}\right)$ with a tracer of dust extinction. We use the H I column density 
Table E.1. Value of $E(B-V) / \tau_{353}$ and $E(B-V) / \mathcal{R}$ obtained by correlation using $E(B-V)$ deduced for each quasar colour.

\begin{tabular}{ccc}
\hline \hline Colour & $E(B-V) / \tau_{353}$ & $\begin{array}{l}E(B-V) / \mathcal{R} \\
{\left[\mathrm{m}^{2} \mathrm{sr} \mathrm{W}^{-1}\right]}\end{array}$ \\
\hline$(g-r)$ & $(1.52 \pm 0.03) \times 10^{4}$ & $(5.53 \pm 0.10) \times 10^{5}$ \\
$(g-i)$ & $(1.47 \pm 0.03) \times 10^{4}$ & $(5.35 \pm 0.10) \times 10^{5}$ \\
$(u-z)$ & $(1.51 \pm 0.03) \times 10^{4}$ & $(5.46 \pm 0.10) \times 10^{5}$ \\
$(u-i)$ & $(1.46 \pm 0.03) \times 10^{4}$ & $(5.28 \pm 0.09) \times 10^{5}$ \\
All & $(1.49 \pm 0.03) \times 10^{4}$ & $(5.40 \pm 0.09) \times 10^{5}$ \\
\hline
\end{tabular}

Notes. The last row is the value obtained by combining all colours (Fig. 22).

provided in the SDSS quasar catalogue as a $\operatorname{proxy}^{23}$ :

$\left(m_{X}-m_{Y}\right)=\psi N_{\mathrm{H} \text { I }}+\left(m_{X 0}-m_{Y 0}\right)$.

The constant term of this linear regression is the average intrinsic colour of quasars but, as quasars are at different redshifts (up to $z \sim 5$ ), this intrinsic colour depends on $z$. Therefore we correlated $\left(m_{X}-m_{Y}\right)$ with $N_{\mathrm{HI}}$ for quasars in bins of $z$ where the width of each bin is set to have 1000 quasars per bin. Figure E. 1 shows the intrinsic colours found as a function of $z$ for the following four colours: $\left(m_{u}-m_{z}\right),\left(m_{u}-m_{i}\right),\left(m_{g}-m_{r}\right)$ and $\left(m_{g}-m_{i}\right)$.

In order to validate the approach we also computed the intrinsic colour as a function of $z$ of the composite quasar spectrum:

$\left(m_{X 0}-m_{Y 0}\right)=-2.5 \log \left(\frac{F_{Y 0}}{F_{X 0}}\right)$.

The results for the same four colours are shown in red in Fig. E.1. The good agreement between the two methods indicates that the composite spectrum is a good average representation. It also gives confidence in its use to estimate $E(B-V)$ using the formalism described earlier.

The correspondence between the two methods is less good at high redshift. This can be attributed to the Lyman- $\alpha$ line that enters the shortest wavelength band. Indeed Mörtsell (2013) mentioned that at $z>2.3$, Lyman- $\alpha$ introduces large variations in the intrinsic quasar colour computed with band $g$. A similar effect is seen at $z>1.7$ for colours using the $u$ band. We also noticed a larger dispersion in the residual $\left(\left(m_{X}-m_{Y}\right)-\psi N_{\mathrm{HI}}\right)$ for bins with $z<0.7$. Based on this, we selected quasars in the redshift range $0.7<z<1.7$. The sample contains 53399 quasars.

For each quasar at a given redshift $z$, we computed the colour excesses $E(X-Y)=\left(m_{X}-m_{Y}\right)-\left(m_{X 0}-m_{Y 0}\right)$ using the intrinsic colour estimated with the correlation with $N_{\mathrm{H}}$ in bins of redshift (Eq. (E.7)). We then integrated numerically Eqs. (E.5) and (E.4) using the composite quasar spectrum redshifted appropriately and solved for $E(B-V)$ using Eq. (E.3). Because we work with colours, the exact normalization of the quasar spectrum $\left(f_{\lambda}\right)$ and of the SDSS transmissions $\left(T_{i}\right)$ cancels out in Eq. (E.3). With this procedure we obtain an estimate of $E(B-V)$ for each quasar independently for each colour excess $E(X-Y)$.

For each quasar position we extracted the values of $\tau_{353}$ and $\mathcal{R}$ and then correlated globally with $E(B-V)$ for each colour $(X-Y)$. Table E. 1 gives the conversion factors $E(B-V) / \tau_{353}$ and $E(B-V) / \mathcal{R}$ estimated for each colour as well as the one obtained by combining all colours, weighted by their uncertainties.

Given the very low value of $E(B-V),<0.1$, the large number of measurements is key here to beat down the noise of the SDSS

\footnotetext{
${ }^{23}$ We preferred not to use the Planck $\tau_{353}$ or the SFD map so as not to bias the analysis.
}

data but also the variations of the quasar spectra around the template spectrum. The intercepts of the regressions of $E(B-V)$ vs. $\tau_{353}$ and $E(B-V)$ vs. $\mathcal{R}$ are small for each colour, indicating that there is a coherence between the zero levels of the Planck and IRAS maps used to build $\tau_{353}$ and the estimate of the intrinsic colours of quasars, both based on a correlation with $N_{\mathrm{HI}}$.

\section{Appendix F: Maps in the Planck Legacy Archive}

Maps of the Planck thermal dust emission model described in this paper can be obtained from the Planck Legacy Archive $(\mathrm{PLA})^{24}$. The maps available give the three MBB parameters $\left(\tau_{353}, T_{\text {obs }}\right.$, and $\left.\beta_{\text {obs }}\right)$ with their uncertainties $\left(\delta \tau_{353}, \delta T_{\text {obs }}\right.$, and $\delta \beta_{\text {obs }}$ ) obtained using data from which point sources were not removed. In addition, the maps of $\mathcal{R}$ (point sources in) and of $E(B-V)_{\text {xgal }}$ are made available; the latter is $\mathcal{R}$ obtained with data from which point sources were removed and scaled to $E(B-V)$ with the conversion factor computed using SDSS quasars.

A first release of the Planck thermal dust model was made available on the PLA in March 2013, together with other 2013 Planck products. This first model is superseded by the model presented in this paper. The first model was made using Planck data from which ZE was not removed, to be compatible with what was used for the cosmological analysis. The $3000 \mathrm{GHz}$ map used in this first edition was the IRIS map. The second release described in this paper is based on Planck data from which ZE was removed and on a combination of IRIS and Schlegel et al. (1998) for the $3000 \mathrm{GHz}$ map.

The impact of this change is significant only in regions of the sky of low Galactic emission and low ecliptic latitude. It affects the parameters $T_{\text {obs }}$ and $\beta_{\text {obs }}$ only slightly, at the $2 \%$ level. Considering the pixels in the low $N_{\mathrm{HI}}$ mask, the average $T_{\mathrm{obs}}$ went from 20.4 to $20.8 \mathrm{~K}$ from the first to the second version, while the average $\beta_{\text {obs }}$ went from 1.59 to 1.55 . That means that the shape of the dust SED is not significantly different from one data set to the other. The main effect of the change of data set is on $\tau_{353}$ because the $\mathrm{ZE}$ removed data have less emission; from the first to the second version, $\left\langle\tau_{353}\right\rangle$ is reduced by about $25 \%$ in the most diffuse areas of the sky, from $1.3 \times 10^{-6}$ to $9.6 \times 10^{-7}$.

A significant difference between the two releases concerns the $E(B-V)$ map. In both releases the calibration was done the same way, using SDSS quasars, but in the first release the $E(B-V)$ map was based on $\tau_{353}$ instead of $\mathcal{R}$. Globally both $E(B-V)$ maps agree but not on small scales where the $353 \mathrm{GHz}$ the CIBA is much more present in $\tau_{353}$ than some spectrally-averaged CIBA is in $\mathcal{R}$. The CIBA in $\tau_{353}$ introduces an additional noise in $E(B-V)$ of the order of 0.003 magnitude. In that respect the $E(B-V)$ map of the second version is a significant improvement.

\section{References}

Abazajian, K. N., Adelman-McCarthy, J. K., Agüeros, M. A., et al. 2009, ApJS, 182,543

Albert, C. E., \& Danly, L. 2004, in High Velocity Clouds, eds. H. van Woerden, B. P. Wakker, U. J. Schwarz, \& K. S. de Boer, 312, 73

Arab, H., Abergel, A., Habart, E., et al. 2012, A\&A, 541, A19

Arce, H. G., \& Goodman, A. A. 1999, ApJ, 512, L135

Battersby, C., Bally, J., Ginsburg, A., et al. 2011, A\&A, 535, A128

Beckwith, S. V. W., Henning, T., \& Nakagawa, Y. 2000, Protostars and Planets IV, 533

Beichman, C. A., Neugebauer, G., Habing, H. J., Clegg, P. E., \& Chester, T. J. 1988, Infrared astronomical satellite (IRAS) catalogs and atlases. Volume 1: Explanatory supplement

\footnotetext{
${ }^{24}$ http://www.sciops.esa.int/index $\cdot$ php?project= planck\&page=Planck_Legacy_Archive
} 
Planck collaboration: Planck 2013 results. XI.

Bennett, C. L., Fixsen, D. J., Hinshaw, G., et al. 1994, ApJ, 434, 587 Bennett, C. L., Larson, D., Weiland, J. L., et al. 2013, ApJS, 208, 20 Birnstiel, T., Klahr, H., \& Ercolano, B. 2012, A\&A, 539, A148 Blain, A. W., Barnard, V. E., \& Chapman, S. C. 2003, MNRAS, 338, 733 Blitz, L., Bazell, D., \& Desert, F. X. 1990, ApJ, 352, L13 Bohlin, R. C., Savage, B. D., \& Drake, J. F. 1978, ApJ, 224, 132 Bolatto, A. D., Wolfire, M., \& Leroy, A. K. 2013, ARA\&A, 51, 207 Boulanger, F., \& Pérault, M. 1988, ApJ, 330, 964

Boulanger, F., Abergel, A., Bernard, J.-P., et al. 1996, A\&A, 312, 256 Brauer, F., Dullemond, C. P., \& Henning, T. 2008, A\&A, 480, 859 Burstein, D., \& Heiles, C. 1978, ApJ, 225, 40

Calabretta, M. R., \& Greisen, E. W. 2002, A\&A, 395, 1077

Cambrésy, L., Boulanger, F., Lagache, G., \& Stepnik, B. 2001, A\&A, 375, 999

Cardelli, J. A., Clayton, G. C., \& Mathis, J. S. 1989, ApJ, 345, 245

Compiègne, M., Verstraete, L., Jones, A., et al. 2011, A\&A, 525, A103

Dale, D. A., Aniano, G., Engelbracht, C. W., et al. 2012, ApJ, 745, 95

Delabrouille, J., Betoule, M., Melin, J.-B., et al. 2013, A\&A, 553, A96

Désert, F. X., Bazell, D., \& Boulanger, F. 1988, ApJ, 334, 815

Désert, F.-X., Macías-Pérez, J. F., Mayet, F., et al. 2008, A\&A, 481, 411

Draine, B. T. 2003, ARA\&A, 41, 241

Draine, B. T., \& Li, A. 2007, ApJ, 657, 810

Dupac, X., Bernard, J.-P., Boudet, N., et al. 2003, A\&A, 404, L11

Finkbeiner, D. P., Davis, M., \& Schlegel, D. J. 1999, ApJ, 524, 867

Fitzpatrick, E. L. 1999, PASP, 111, 63

Fitzpatrick, E. L., \& Massa, D. 2005, AJ, 130, 1127

Gillmon, K., Shull, J. M., Tumlinson, J., \& Danforth, C. 2006, ApJ, 636, 891

Goodman, A. A., Pineda, J. E., \& Schnee, S. L. 2009, ApJ, 692, 91

Górski, K. M., Hivon, E., Banday, A. J., et al. 2005, ApJ, 622, 759

Grenier, I. A., Casandjian, J.-M., \& Terrier, R. 2005, Science, 307, 1292

Heiles, C., Reach, W. T., \& Koo, B.-C. 1988, ApJ, 332, 313

Hildebrand, R. H. 1983, QJRAS, 24, 267

Hinshaw, G., Weiland, J. L., Hill, R. S., et al. 2009, ApJS, 180, 225

Jones, D. O., West, A. A., \& Foster, J. B. 2011, AJ, 142, 44

Juvela, M., \& Ysard, N. 2012a, A\&A, 541, A33

Juvela, M., \& Ysard, N. 2012b, A\&A, 539, A71

Juvela, M., Montillaud, J., Ysard, N., \& Lunttila, T. 2013, A\&A, 556, A63

Kainulainen, J., Federrath, C., \& Henning, T. 2013, A\&A, 553, L8

Kalberla, P. M. W., Burton, W. B., Hartmann, D., et al. 2005, A\&A, 440, 775

Kelly, B. C., Shetty, R., Stutz, A. M., et al. 2012, ApJ, 752, 55

Kelsall, T., Weiland, J. L., Franz, B. A., et al. 1998, ApJ, 508, 44

Knapp, G. R., \& Kerr, F. J. 1974, A\&A, 35, 361

Köhler, M., Guillet, V., \& Jones, A. 2011, A\&A, 528, A96

Köhler, M., Stepnik, B., Jones, A. P., et al. 2012, A\&A, 548, A61

Lagache, G., Abergel, A., Boulanger, F., Désert, F. X., \& Puget, J.-L. 1999, A\&A, 344, 322

Lagache, G., Haffner, L. M., Reynolds, R. J., \& Tufte, S. L. 2000, A\&A, 354, 247

Launhardt, R., Stutz, A. M., Schmiedeke, A., et al. 2013, A\&A, 551, A98

Lebrun, F., Paul, J. A., Bignami, G. F., et al. 1982, A\&A, 107, 390

Lilley, A. E. 1955, ApJ, 121, 559

Liszt, H. 2014, ApJ, 780, 10

Lockman, F. J., \& Condon, J. J. 2005, AJ, 129, 1968

Lombardi, M., Alves, J., \& Lada, C. J. 2011, A\&A, 535, A16

Markwardt, C. B. 2009, in Astronomical Data Analysis Software and Systems XVIII, eds. D. A. Bohlender, D. Durand, \& P. Dowler, 411, 251

Martin, P. G., Roy, A., Bontemps, S., et al. 2012, ApJ, 751, 28

Mathis, J. S., Rumpl, W., \& Nordsieck, K. H. 1977, ApJ, 217, 425

Mathis, J. S., Mezger, P. G., \& Panagia, N. 1983, A\&A, 128, 212

McClure-Griffiths, N. M., Pisano, D. J., Calabretta, M. R., et al. 2009, ApJS, 181,398

Meny, C., Gromov, V., Boudet, N., et al. 2007, A\&A, 468, 171

Miville-Deschênes, M. 2011, in ASP Conf. Ser. 449, eds. P. Bastien, N. Manset, D. P. Clemens, \& N. St-Louis, 187

Miville-Deschênes, M.-A., \& Lagache, G. 2005, ApJS, 157, 302

Miville-Deschênes, M.-A., Lagache, G., Boulanger, F., \& Puget, J.-L. 2007, A\&A, 469, 595

Mörtsell, E. 2013, A\&A, 550, A80

Moshir, M., Kopman, G., \& Conrow, T. A. O. 1992, IRAS Faint Source Survey, Explanatory supplement version 2

O’Donnell, J. E. 1994, ApJ, 422, 158

Ormel, C. W., Min, M., Tielens, A. G. G. M., Dominik, C., \& Paszun, D. 2011, A\&A, 532, A43

Ossenkopf, V., \& Henning, T. 1994, A\&A, 291, 943

Paradis, D., Veneziani, M., Noriega-Crespo, A., et al. 2010, A\&A, 520, L8

Paradis, D., Paladini, R., Noriega-Crespo, A., et al. 2012, A\&A, 537, A113

Peek, J. E. G., \& Graves, G. J. 2010, ApJ, 719, 415

Pénin, A., Lagache, G., Noriega-Crespo, A., et al. 2012, A\&A, 543, A123

Peretto, N., André, P., Könyves, V., et al. 2012, A\&A, 541, A63
Pineda, J. E., Caselli, P., \& Goodman, A. A. 2008, ApJ, 679, 481

Planck Collaboration VII. 2011, A\&A, 536, A7

Planck Collaboration XVII. 2011, A\&A, 536, A17

Planck Collaboration XVIII. 2011, A\&A, 536, A18

Planck Collaboration XIX. 2011, A\&A, 536, A19

Planck Collaboration XXI. 2011, A\&A, 536, A21

Planck Collaboration XXIII. 2011, A\&A, 536, A23

Planck Collaboration XXIV. 2011, A\&A, 536, A24

Planck Collaboration XXV. 2011, A\&A, 536, A25

Planck Collaboration I. 2014, A\&A, 571, A1

Planck Collaboration II. 2014, A\&A, 571, A2

Planck Collaboration III. 2014, A\&A, 571, A3

Planck Collaboration IV. 2014, A\&A, 571, A4

Planck Collaboration V. 2014, A\&A, 571, A5

Planck Collaboration VI. 2014, A\&A, 571, A6

Planck Collaboration VII. 2014, A\&A, 571, A7

Planck Collaboration VIII. 2014, A\&A, 571, A8

Planck Collaboration IX. 2014, A\&A, 571, A9

Planck Collaboration X. 2014, A\&A, 571, A10

Planck Collaboration XI. 2014, A\&A, 571, A11

Planck Collaboration XII. 2014, A\&A, 571, A12

Planck Collaboration XIII. 2014, A\&A, 571, A13

Planck Collaboration XIV. 2014, A\&A, 571, A14

Planck Collaboration XV. 2014, A\&A, 571, A15

Planck Collaboration XVI. 2014, A\&A, 571, A16

Planck Collaboration XVII. 2014, A\&A, 571, A17

Planck Collaboration XVIII. 2014, A\&A, 571, A18

Planck Collaboration XIX. 2014, A\&A, 571, A19

Planck Collaboration XX. 2014, A\&A, 571, A20

Planck Collaboration XXI. 2014, A\&A, 571, A21

Planck Collaboration XXII. 2014, A\&A, 571, A22

Planck Collaboration XXIII. 2014, A\&A, 571, A23

Planck Collaboration XXIV. 2014, A\&A, 571, A24

Planck Collaboration XXV. 2014, A\&A, 571, A25

Planck Collaboration XXVI. 2014, A\&A, 571, A26

Planck Collaboration XXVII. 2014, A\&A, 571, A27

Planck Collaboration XXVIII. 2014, A\&A, 571, A28

Planck Collaboration XXIX. 2014, A\&A, 571, A29

Planck Collaboration XXX. 2014, A\&A, 571, A30

Planck Collaboration XXXI. 2014, A\&A, 571, A31

Planck Collaboration Int. XIV. 2014, A\&A, 564, A45

Planck Collaboration Int. XVII. 2014, A\&A, 566, A55

Puget, J.-L., Abergel, A., Bernard, J.-P., et al. 1996, A\&A, 308, L5

Rachford, B. L., Snow, T. P., Tumlinson, J., et al. 2002, ApJ, 577, 221

Rachford, B. L., Snow, T. P., Destree, J. D., et al. 2009, ApJS, 180, 125

Reach, W. T., Koo, B.-C., \& Heiles, C. 1994, ApJ, 429, 672

Reach, W. T., Wall, W. F., \& Odegard, N. 1998, ApJ, 507, 507

Roy, A., Martin, P. G., Polychroni, D., et al. 2013, ApJ, 763, 55

Ryter, C., Cesarsky, C. J., \& Audouze, J. 1975, ApJ, 198, 103

Savage, B. D., \& Jenkins, E. B. 1972, ApJ, 172, 491

Schlafly, E. F., \& Finkbeiner, D. P. 2011, ApJ, 737, 103

Schlafly, E. F., Finkbeiner, D. P., Schlegel, D. J., et al. 2010, ApJ, 725, 1175

Schlegel, D. J., Finkbeiner, D. P., \& Davis, M. 1998, ApJ, 500, 525 (SFD)

Schnee, S., Kauffmann, J., Goodman, A., \& Bertoldi, F. 2007, ApJ, 657, 838

Schnee, S., Li, J., Goodman, A. A., \& Sargent, A. I. 2008, ApJ, 684, 1228

Schneider, D. P., Richards, G. T., Hall, P. B., et al. 2010, AJ, 139, 2360

Schneider, N., Bontemps, S., Simon, R., et al. 2011, A\&A, 529, A1

Shetty, R., Kauffmann, J., Schnee, S., \& Goodman, A. A. 2009a, ApJ, 696, 676

Shetty, R., Kauffmann, J., Schnee, S., Goodman, A. A., \& Ercolano, B. 2009b, ApJ, 696, 2234

Skrutskie, M. F., Cutri, R. M., Stiening, R., et al. 2006, AJ, 131, 1163

Stepnik, B., Abergel, A., Bernard, J.-P., et al. 2003, A\&A, 398, 551

Vanden Berk, D. E., Richards, G. T., Bauer, A., et al. 2001, AJ, 122, 549

Veneziani, M., Ade, P. A. R., Bock, J. J., et al. 2010, ApJ, 713, 959

Veneziani, M., Piacentini, F., Noriega-Crespo, A., et al. 2013, ApJ, 772, 56

Wakker, B. P. 2006, ApJS, 163, 282

Weingartner, J. C., \& Draine, B. T. 2001, ApJ, 548, 296

Ysard, N., Juvela, M., Demyk, K., et al. 2012, A\&A, 542, A21

1 APC, AstroParticule et Cosmologie, Université Paris Diderot, CNRS/IN2P3, CEA/lrfu, Observatoire de Paris, Sorbonne Paris Cité, 10 rue Alice Domon et Léonie Duquet, 75205 Paris Cedex 13, France

2 Aalto University Metsähovi Radio Observatory and Dept of Radio Science and Engineering, PO Box 13000, 00076 AALTO, Finland 
3 African Institute for Mathematical Sciences, 6-8 Melrose Road, Muizenberg, Cape Town, South Africa

4 Agenzia Spaziale Italiana Science Data Center, via del Politecnico snc, 00133 Roma, Italy

5 Agenzia Spaziale Italiana, Viale Liegi 26, Roma, Italy

6 Astrophysics Group, Cavendish Laboratory, University of Cambridge, J J Thomson Avenue, Cambridge CB3 0HE, UK

7 Astrophysics \& Cosmology Research Unit, School of Mathematics, Statistics \& Computer Science, University of KwaZulu-Natal, Westville Campus, Private Bag X54001, 4000 Durban, South Africa

8 CITA, University of Toronto, 60 St. George St., Toronto, ON M5S 3H8, Canada

9 CNRS, IRAP, 9 Av. colonel Roche, BP 44346, 31028 Toulouse Cedex 4, France

${ }^{10}$ California Institute of Technology, Pasadena, California, USA

11 Centre for Theoretical Cosmology, DAMTP, University of Cambridge, Wilberforce Road, Cambridge CB3 0WA, UK

12 Centro de Estudios de Física del Cosmos de Aragón (CEFCA), Plaza San Juan 1, planta 2, 44001 Teruel, Spain

13 Computational Cosmology Center, Lawrence Berkeley National Laboratory, Berkeley, California, USA

14 Consejo Superior de Investigaciones Científicas (CSIC), Madrid, Spain

15 DSM/Irfu/SPP, CEA-Saclay, 91191 Gif-sur-Yvette Cedex, France

16 DTU Space, National Space Institute, Technical University of Denmark, Elektrovej 327, 2800 Kgs. Lyngby, Denmark

17 Département de Physique Théorique, Université de Genève, 24 Quai E. Ansermet, 1211 Genève 4, Switzerland

18 Département de physique, de génie physique et d'optique, Université Laval, Québec, Canada

19 Departamento de Física Fundamental, Facultad de Ciencias, Universidad de Salamanca, 37008 Salamanca, Spain

20 Departamento de Física, Universidad de Oviedo, Avda. Calvo Sotelo $\mathrm{s} / \mathrm{n}$, Oviedo, Spain

21 Department of Astronomy and Astrophysics, University of Toronto, 50 Saint George Street, Toronto, Ontario, Canada

22 Department of Astrophysics/IMAPP, Radboud University Nijmegen, PO Box 9010, 6500 GL Nijmegen, The Netherlands

23 Department of Electrical Engineering and Computer Sciences, University of California, Berkeley, California, USA

24 Department of Physics \& Astronomy, University of British Columbia, 6224 Agricultural Road, Vancouver, British Columbia, Canada

25 Department of Physics and Astronomy, Dana and David Dornsife College of Letter, Arts and Sciences, University of Southern California, Los Angeles, CA 90089, USA

26 Department of Physics and Astronomy, University College London, London WC1E 6BT, UK

27 Department of Physics, Florida State University, Keen Physics Building, 77 Chieftan Way, Tallahassee, Florida, USA

28 Department of Physics, Gustaf Hällströmin katu 2a, University of Helsinki, Helsinki, Finland

29 Department of Physics, Princeton University, Princeton, New Jersey, USA

30 Department of Physics, University of California, One Shields Avenue, Davis, California, USA

31 Department of Physics, University of California, Santa Barbara, California, USA

32 Department of Physics, University of Illinois at Urbana-Champaign, 1110 West Green Street, Urbana, Illinois, USA

33 Dipartimento di Fisica e Astronomia G. Galilei, Università degli Studi di Padova, via Marzolo 8, 35131 Padova, Italy

34 Dipartimento di Fisica e Scienze della Terra, Università di Ferrara, via Saragat 1, 44122 Ferrara, Italy

35 Dipartimento di Fisica, Università La Sapienza, P.le A. Moro 2, Roma, Italy

36 Dipartimento di Fisica, Università degli Studi di Milano, via Celoria, 16, Milano, Italy

37 Dipartimento di Fisica, Università degli Studi di Trieste, via A. Valerio 2, Trieste, Italy
38 Dipartimento di Fisica, Università di Roma Tor Vergata, via della Ricerca Scientifica 1, Roma, Italy

39 Discovery Center, Niels Bohr Institute, Blegdamsvej 17, Copenhagen, Denmark

40 Dpto. Astrofísica, Universidad de La Laguna (ULL), 38206 La Laguna, Tenerife, Spain

${ }^{41}$ European Space Agency, ESAC, Planck Science Office, Camino bajo del Castillo, s/n, Urbanización Villafranca del Castillo, Villanueva de la Cañada, Madrid, Spain

42 European Space Agency, ESTEC, Keplerlaan 1, 2201 AZ Noordwijk, The Netherlands

43 Finnish Centre for Astronomy with ESO (FINCA), University of Turku, Väisäläntie 20, 21500 Piikkiö, Finland

${ }^{44}$ Helsinki Institute of Physics, Gustaf Hällströmin katu 2, University of Helsinki, Helsinki, Finland

45 INAF-Osservatorio Astrofisico di Catania, via S. Sofia 78, Catania, Italy

46 INAF-Osservatorio Astronomico di Padova, Vicolo dell' Osservatorio 5, Padova, Italy

47 INAF-Osservatorio Astronomico di Roma, via di Frascati 33, Monte Porzio Catone, Italy

48 INAF-Osservatorio Astronomico di Trieste, via G.B. Tiepolo 11, Trieste, Italy

49 INAF Istituto di Radioastronomia, via P. Gobetti 101, 40129 Bologna, Italy

50 INAF/IASF Bologna, via Gobetti 101, Bologna, Italy

51 INAF/IASF Milano, via E. Bassini 15, Milano, Italy

${ }^{52}$ INFN, Sezione di Bologna, via Irnerio 46, 40126, Bologna, Italy

53 INFN, Sezione di Roma 1, Università di Roma Sapienza, Piazzale Aldo Moro 2, 00185, Roma, Italy

${ }^{54}$ INFN/National Institute for Nuclear Physics, via Valerio 2, 34127 Trieste, Italy

55 IPAG: Institut de Planétologie et d'Astrophysique de Grenoble, Université Joseph Fourier, Grenoble 1 / CNRS-INSU, UMR 5274, 38041 Grenoble, France

56 ISDC Data Centre for Astrophysics, University of Geneva, ch. d'Ecogia 16, Versoix, Switzerland

57 IUCAA, Post Bag 4, Ganeshkhind, Pune University Campus, 411007 Pune, India

58 Imperial College London, Astrophysics group, Blackett Laboratory, Prince Consort Road, London, SW7 2AZ, UK

59 Infrared Processing and Analysis Center, California Institute of Technology, Pasadena, CA 91125, USA

${ }^{60}$ Institut Néel, CNRS, Université Joseph Fourier Grenoble I, 25 rue des Martyrs, Grenoble, France

61 Institut Universitaire de France, 103 Bd Saint-Michel, 75005, Paris, France

${ }^{62}$ Institut d'Astrophysique Spatiale, CNRS (UMR8617) Université Paris-Sud 11, Bâtiment 121, Orsay, France

63 Institut d'Astrophysique de Paris, CNRS (UMR7095), 98bis Bd Arago, 75014 Paris, France

${ }^{64}$ Institute for Space Sciences, Bucharest-Magurale, Romania

65 Institute of Astronomy and Astrophysics, Academia Sinica, Taipei, Taiwan

${ }^{66}$ Institute of Astronomy, University of Cambridge, Madingley Road, Cambridge CB3 0HA, UK

${ }^{67}$ Institute of Theoretical Astrophysics, University of Oslo, Blindern, Oslo, Norway

68 Instituto de Astrofísica de Canarias, C/Vía Láctea s/n, La Laguna, Tenerife, Spain

69 Instituto de Física de Cantabria (CSIC-Universidad de Cantabria), Avda. de los Castros s/n, Santander, Spain

70 Jet Propulsion Laboratory, California Institute of Technology, 4800 Oak Grove Drive, Pasadena, California, USA

71 Jodrell Bank Centre for Astrophysics, Alan Turing Building, School of Physics and Astronomy, The University of Manchester, Oxford Road, Manchester, M13 9PL, UK

72 Kavli Institute for Cosmology Cambridge, Madingley Road, Cambridge, CB3 OHA, UK

${ }^{73}$ LAL, Université Paris-Sud, CNRS/IN2P3, Orsay, France 
74 LERMA, CNRS, Observatoire de Paris, 61 Avenue de l'Observatoire, Paris, France

75 Laboratoire AIM, IRFU/Service d'Astrophysique - CEA/DSM CNRS - Université Paris Diderot, Bât. 709, CEA-Saclay, 91191 Gifsur-Yvette Cedex, France

76 Laboratoire Traitement et Communication de l'Information, CNRS (UMR 5141) and Télécom ParisTech, 46 rue Barrault, 75634 Paris Cedex 13, France

77 Laboratoire de Physique Subatomique et de Cosmologie, Université Joseph Fourier Grenoble I, CNRS/IN2P3, Institut National Polytechnique de Grenoble, 53 rue des Martyrs, 38026 Grenoble Cedex, France

78 Laboratoire de Physique Théorique, Université Paris-Sud 11 \& CNRS, Bâtiment 210, 91405 Orsay, France

79 Lawrence Berkeley National Laboratory, Berkeley, California, USA

${ }^{80}$ Max-Planck-Institut für Astrophysik, Karl-Schwarzschild-Str. 1, 85741 Garching, Germany

81 McGill Physics, Ernest Rutherford Physics Building, McGill University, 3600 rue University, Montréal, QC, H3A 2T8, Canada

82 MilliLab, VTT Technical Research Centre of Finland, Tietotie 3, Espoo, Finland

83 National University of Ireland, Department of Experimental Physics, Maynooth, Co. Kildare, Ireland

${ }^{84}$ Niels Bohr Institute, Blegdamsvej 17, Copenhagen, Denmark

85 Observational Cosmology, Mail Stop 367-17, California Institute of Technology, Pasadena, CA, 91125, USA

${ }^{86}$ Optical Science Laboratory, University College London, Gower Street, London, UK
87 Princeton University Observatory, Peyton Hall, Princeton, NJ 08544-1001, USA

88 SB-ITP-LPPC, EPFL, 1015, Lausanne, Switzerland

89 SISSA, Astrophysics Sector, via Bonomea 265, 34136 Trieste, Italy

90 School of Physics and Astronomy, Cardiff University, Queens Buildings, The Parade, Cardiff, CF24 3AA, UK

91 Space Research Institute (IKI), Russian Academy of Sciences, Profsoyuznaya Str, 84/32, 117997 Moscow, Russia

92 Space Sciences Laboratory, University of California, Berkeley, California, USA

93 Special Astrophysical Observatory, Russian Academy of Sciences, Nizhnij Arkhyz, Zelenchukskiy region, 369167 KarachaiCherkessian Republic, Russia

94 Stanford University, Dept of Physics, Varian Physics Bldg, 382 via Pueblo Mall, Stanford, California, USA

95 Sub-Department of Astrophysics, University of Oxford, Keble Road, Oxford OX1 3RH, UK

96 Theory Division, PH-TH, CERN, 1211 Geneva 23, Switzerland

97 UPMC Univ. Paris 06, UMR7095, 98bis Boulevard Arago, 75014 Paris, France

98 Université de Toulouse, UPS-OMP, IRAP, 31028 Toulouse Cedex 4, France

99 Universities Space Research Association, Stratospheric Observatory for Infrared Astronomy, MS 232-11, Moffett Field, CA 94035, USA

100 University of Granada, Departamento de Física Teórica y del Cosmos, Facultad de Ciencias, Granada, Spain

101 Warsaw University Observatory, Aleje Ujazdowskie 4, 00-478 Warszawa, Poland 